\title{
A NEW CONSTRUCTION OF CYCLIC HOMOLOGY
}

\author{
VICTOR GINZBURG AND TRAVIS SCHEDLER
}

\begin{abstract}
Based on the ideas of Cuntz and Quillen, we give a simple construction of cyclic homology of unital algebras in terms of the noncommutative de Rham complex and a certain differential similar to the equivariant de Rham differential. We describe the Connes exact sequence in this setting.

We define equivariant Deligne cohomology and construct, for each $n \geq 1$, a natural map from cyclic homology of an algebra to the $\mathrm{GL}_{n}$-equivariant Deligne cohomology of the variety of $n$-dimensional representations of that algebra. The bridge between cyclic homology and equivariant Deligne cohomology is provided by extended cyclic homology, which we define and compute here, based on the extended noncommutative de Rham complex introduced previously by the authors.
\end{abstract}

\section{CONTENTS}

1. Introduction

2. Noncommutative differential forms

3. Main constructions

4. The Connes exact sequence

5. Extended Karoubi-de Rham complex and cyclic homology

6. Proof of Theorem5.3.10

7. Periodic and negative extended cyclic homology

8. The representation functor and equivariant Deligne cohomology

References

\section{INTRODUCTION}

1.1. There are several definitions of cyclic homology. The original definition, due to A. Connes [Con] (see also Tsygan [Tsy1]), works for algebras over fields of characteristic zero and is based on a cyclic version of the Hochschild complex. Another definition that involves a double complex and that works in arbitrary characteristic is due to Loday and Quillen [LQ] (motivated by Tsygan [Tsy1]). This definition may be reformulated in terms of a slightly different (b, B)-complex, where $\mathrm{b}$ and $\mathrm{B}$ are the Hochschild and the Connes differentials, respectively. There is yet another definition of cyclic homology as a certain nonabelian derived functor, due to Feigin and Tsygan [FT]. All the above definitions are known to be equivalent (see, e.g., [Lo]), and each one has certain advantages.

In this paper, we give one more definition of (reduced) cyclic homology for unital algebras over fields of characteristic zero (more generally, over commutative rings $\mathbf{k}$ containing $\mathbb{Q}$, when the algebra is k-split). Our approach is partly motivated by a well known analogy, that goes back to Rinehart [Ri], between the Connes differential $B$ and the familiar de Rham differential on differential forms. In the case of commutative algebras, this analogy can be made precise (see, e.g., [Lo, §3.4]). In the general case of not necessarily commutative algebras, Karoubi [Kar] introduced (motivated by an earlier construction of Connes) a certain noncommutative de Rham complex that comes equipped with a natural differential d, the Karoubi-de Rham differential. The cohomology of the noncommutative de Rham complex is not directly related to cyclic homology, however. 
A precise relation between the two is given by the so-called Connes-Karoubi theorem; see [Lo, Theorem 2.6.7] or (4.1.2) below.

In our approach, cyclic homology is constructed directly in terms of the noncommutative de Rham complex so that the Karoubi-de Rham differential d literally replaces the Connes differential $B$. There is also another differential involved in the construction, a counterpart of the Hochschild differential b. This new differential i, introduced first in [CBEG, $\S 2.8, \S 3.1$ ], anti-commutes with d (while b does not anti-commute with $\mathrm{d}$ ) and is, we believe, more closely related to geometry than b. Specifically, for every (not necessarily commutative) algebra $A$, there is a canonical derivation $\Delta: A \rightarrow A \otimes A$ given by the formula $\Delta(a):=1 \otimes a-a \otimes 1$. The differential i may be thought of as a contraction operation of noncommutative differential forms with the derivation $\Delta$; see op. cit., [GS], and Section 5 below for more details.

There are two features of the construction of cyclic homology given in this paper which, we believe, are especially appealing. The first one is its close analogy with equivariant cohomology. A well known result of Goodwillie [Go] says that the cyclic homology of (the de Rham DG algebra of) a manifold $X$ is isomorphic to the $S^{1}$-equivariant cohomology of $L(X)$, the corresponding free loop space. The role of the extended noncommutative de Rham complex, introduced in $\$ 5$ below, is somewhat analogous to that of the equivariant de Rham complex of the free loop space. Specifically, our extended noncommutative de Rham complex comes equipped with a pair of anti-commuting differentials, the Karoubi-de Rham differential $d$ and a differential $i_{t}$ which is analogous to the $S^{1}$-equivariant differential. Cyclic homology is then related to the cohomology of the extended noncommutative de Rham complex via a map, which we call the 'Tsygan map', that intertwines the differentials $b$ and $B$, respectively, in the standard (b, B)-complex with the differentials $i_{t}$ and $d$, respectively, in the extended noncommutative de Rham complex. The Tsygan map may be viewed as a noncommutative analogue of multiplication by the function $\exp (t)$.

The second feature of our construction is its obvious similarity with truncated de Rham complexes. The latter show up in the standard description of cyclic homology of the coordinate ring of an algebraic variety. Thus, our construction provides an analogous description in the noncommutative setting. Some of the above mentioned analogies can be made precise via the notion of representation functor and equivariant Deligne cohomology. This will be discussed in 8 .

1.2. Layout of the paper. In $\S 2$, we introduce the noncommutative de Rham complex and discuss harmonic decomposition, a powerful technical tool discovered by Cuntz and Quillen [CQ1], [CQ2].

In $\$ 3$, we give our new construction of cyclic homology, as well as similar constructions for periodic and negative cyclic homology. We then use harmonic decomposition to prove that our construction and the standard one are equivalent.

Section 4 is devoted to the Connes exact sequence. We show how to construct such a sequence entirely within our approach. We also establish the equivalence with the standard construction.

Section 5 contains some of the most important results of the paper. We introduce the extended noncommutative de Rham complex and define extended cyclic homology. Extended cyclic homology groups come equipped with an additional 'weight grading.' We show that the positive weight part is isomorphic to the Karoubi-de Rham homology, while the negative weight part reduces to the usual cyclic homology. A key role in proving these results is played by the Tsygan map. We have arrived at the definition of the Tsygan map by analyzing Tsygan's construction [Tsy2] (that construction was motivated, in turn, by our results in [GS]).

Section 6 is devoted to the proof of Theorem 5.3.10, which is perhaps the most difficult result of the paper. In \$7, we discuss 'extended versions' of periodic and negative cyclic homology.

In the last section we relate our results to geometry. We begin by introducing equivariant Deligne cohomology, an equivariant counterpart of Deligne cohomology, i.e., of the cohomology theory 
based on truncated algebraic de Rham complexes. The equivariant Deligne cohomology theory that we define appears to be new.

Next, we give a short review of some standard constructions related to the representation functor. Recall that, associated with a (not necessarily commutative) algebra $A$ and an integer $n \geq 1$, there is an affine scheme $\operatorname{Rep}_{n} A$ parametrizing $n$-dimensional representations of $A$, cf. [CBEG]. This scheme comes equipped with a natural action of the algebraic group $\mathrm{GL}_{n}$, by base change transformations. An important application of our approach is a construction of a canonical map from the cyclic homology of $A$ to the equivariant Deligne cohomology of the scheme $\operatorname{Rep}_{n} A$. We should point out that considering equivariant (as opposed to ordinary) cohomology of representation schemes is, in a sense, the only natural thing to do. Indeed, two representations of the algebra $A$ are equivalent if and only they belong to the same $\mathrm{GL}_{n}$-orbit in $\operatorname{Rep}_{n} A$. Thus, the object which is most relevant here is the quotient stack $\operatorname{Rep}_{n} A / \mathrm{GL}_{n}$ rather than the scheme $\operatorname{Rep}_{n} A$ itself. The $\mathrm{GL}_{n}$-equivariant Deligne cohomology of the scheme $\operatorname{Rep}_{n} A$ may be viewed as the ordinary Deligne cohomology of the stack $\operatorname{Rep}_{n} A / \mathrm{GL}_{n}$.

In the special case where $n=1$ and $A$ is the coordinate ring of a smooth affine algebraic variety $X$, one has $\operatorname{Rep}_{1} A=X$. In this case, our construction reduces to, and provides a more explicit form of, the well known isomorphism between cyclic homology of the coordinate ring and Deligne cohomology of $X$, cf. [Lo, §3.6].

A different relation between cyclic homology of an algebra and its representation schemes is studied in the recent paper by Berest, Khachatryan, and A. Ramadoss [BKR]. It does not consider equivariant cohomology, however.

1.3. Acknowledgements. We are very much indebted to Boris Tsygan for his kind explanations of the construction in [Tsy2], which inspired the present work.

The first author was supported in part by the NSF grant DMS-1001677. The second author is a five-year fellow of the American Institute of Mathematics, and was partially supported by the ARRA-funded NSF grant DMS-0900233.

\section{NONCOMMUTATIVE DIFFERENTIAL FORMS}

2.1. The Karoubi-de Rham complex. Fix $\mathbf{k}$, a unital commutative $\mathbb{Q}$-algebra, and put $\otimes=\otimes_{\mathbf{k}}$.

Throughout the paper, we fix a unital associative algebra $A$ over the ground ring $\mathrm{k}$ satisfying the additional assumption that the natural map $\mathbf{k} \rightarrow A$ is a $\mathbf{k}$-split injection, i.e., there exists a k-module direct sum decomposition $A=\mathbf{k} \oplus \bar{A}$, where $\bar{A}=A / \mathbf{k}$. This assumption trivially holds if $\mathbf{k}$ is a field.

Associated with $A$, there is a dg algebra $(\Omega A, \mathrm{~d})$ of noncommutative differential forms, the differential envelope of $A$ [CQ1]. By definition, $\Omega A$ is the quotient of $T_{\mathbf{k}}(A \oplus \bar{A})$, a free tensor algebra of the k-module $A \oplus \bar{A}$, by the relations of the form $a \otimes b=a b$ and $\mathrm{d}(a b)=\mathrm{d} a \otimes b+a \otimes \mathrm{d} b$, for all $a, b \in A$, where we use the notation $\mathrm{d} a$ for the class of $a \in A$ in $\bar{A}$. One puts a grading $\Omega^{\bullet} A=\bigoplus_{n \geq 0} \Omega^{n} A$, on $\Omega A$, by placing $A$ in degree 0 and $\bar{A}$ in degree 1 . The differential $\mathrm{d}: \Omega^{\bullet} A \rightarrow \Omega^{\bullet+1} A$ is given by the obvious assignment: $a \mapsto \mathrm{d} a \mapsto 0$. This differential kills $\mathbf{k} \subset \Omega^{0} A$ and hence descends to $\bar{\Omega} A:=\Omega A / \mathbf{k}$, the reduced complex.

As explained in [CQ1, §1], one has a canonical isomorphism of left $A$-modules

$$
A \otimes \bar{A}^{\otimes n} \stackrel{\sim}{\rightarrow} \Omega^{n} A, \quad a_{0} \otimes \mathrm{d} a_{1} \otimes \mathrm{d} a_{2} \otimes \cdots \otimes \mathrm{d} a_{n} \mapsto a_{0} \mathrm{~d} a_{1} \mathrm{~d} a_{2} \cdots \mathrm{d} a_{n}, \quad a_{0}, \ldots, a_{n} \in A .
$$

From this and the assumption that $\mathbf{k} \hookrightarrow A$ is split it follows that $(\bar{\Omega} A, \mathbf{d})$ is acyclic; see [CQ1, §1].

For every graded algebra $B$ and graded vector subspace $V \subseteq B$, let $[V, V]$ denote the linear span of (super)commutators $\left[v_{1}, v_{2}\right], v_{1}, v_{2} \in V$. Following Karoubi [Kar], define the noncommutative 
de Rham complex of $A$ and its reduced version by

$$
\operatorname{DR} A:=\Omega A /[\Omega A, \Omega A], \quad \text { and } \quad \overline{\mathrm{DR}} A:=(\operatorname{DR} A) / \mathbf{k}=\bar{\Omega} A / \overline{[\Omega A, \Omega A]} .
$$

We write $f_{\natural}$ for the image of an element $f \in \Omega A$ under the natural projection $\Omega A \rightarrow \mathrm{DR} A$.

The differential d on $\Omega A$ descends to $\mathrm{DR} A$. Let $\operatorname{HD}_{n}(A)$ and $\overline{\mathrm{HD}}_{n}(A)$ denote the $n$-th homology of (DR $A, \mathrm{~d})$ and $(\overline{\mathrm{DR}} A, \mathrm{~d})$, respectively. We will call these the Karoubi-de Rham homology and its reduced version.

Following [CQ1, CQ2], the Hochschild differential on $\Omega A$ (or on $\bar{\Omega} A$ ) is given by the formula

$$
\mathrm{b}(\alpha \mathrm{d} a)=(-1)^{n-1}[\alpha, a], \quad \alpha \in \Omega^{n} A, a \in A, \text { and }\left.\mathrm{b}\right|_{\Omega^{0} A}=0 .
$$

A crucial role below will also be played by another map i : $\Omega A \rightarrow \Omega A$, defined by

$$
\mathbf{i}\left(a_{0} \mathrm{~d} a_{1} \cdots \mathrm{d} a_{n}\right)=\sum_{\ell=1}^{n}(-1)^{(\ell-1)(n-1)+1}\left[a_{\ell}, \mathrm{d} a_{\ell+1} \cdots \mathrm{d} a_{n} a_{0} \mathrm{~d} a_{1} \cdots \mathrm{d} a_{\ell-1}\right] \in\left[A, \Omega^{n-1} A\right] .
$$

It is straightforward to check that the map $\mathbf{i}$ vanishes on $[\Omega A, \Omega A]$ and hence descends to a well defined map $\mathrm{i}: \mathrm{DR}^{\bullet} A \rightarrow \Omega^{\bullet-1} A$. It follows that $\mathbf{i}^{2}=0$. In addition, one can check that $\mathrm{d} \mathrm{i}+\mathrm{i} \mathrm{d}=0$.

The map i was first introduced in [CBEG]. A more conceptual definition of this map (see $\$ 5$ ) was discovered in [GS]. From that definition the above stated properties become immediate consequences of noncommutative calculus.

2.2. Harmonic decomposition. Since the algebra $A$ is fixed throughout the paper, we will often use simplified notation $\Omega=\Omega A, \bar{\Omega}=\bar{\Omega} A, \mathrm{DR}=\mathrm{DR} A$, etc.

Following [Kar], define the Karoubi operator as

$$
\kappa: \bar{\Omega} \rightarrow \bar{\Omega}, \quad \kappa(\alpha \mathrm{d} a)=(-1)^{|\alpha|} \mathrm{d} a \alpha, \quad \alpha \in \Omega, a \in A ;\left.\quad \kappa\right|_{\bar{\Omega}^{0}}=0 .
$$

The Karoubi operator is related to the operators $b$ and $d$ by [Kar, CQ1]

$$
b d+d b=I d-\kappa .
$$

According to [CQ2, §2], there is a direct sum decomposition

$$
\bar{\Omega}=P \bar{\Omega} \oplus P^{\perp} \bar{\Omega}, \quad P \bar{\Omega}:=\operatorname{ker}(\operatorname{Id}-\kappa)^{2}, \quad P^{\perp} \bar{\Omega}:=\operatorname{im}(\operatorname{Id}-\kappa)^{2} .
$$

Let $P$ and $P^{\perp}$ denote the projections onto the first and second summands of this decomposition, respectively, which we will call the harmonic and antiharmonic parts.

It follows from (2.2.2) that $\kappa$ commutes with $\mathrm{d}$ and $\mathrm{b}$. Hence, harmonic decomposition is stable under $\kappa, \mathrm{b}$, and $\mathrm{d}$, and it induces a similar decomposition on $H(\Omega, \mathrm{b})$ and $H(\Omega, \mathrm{d})$.

Let $\mathrm{N}$ be the grading operator on $\bar{\Omega}$, defined by $\left.\mathrm{N}\right|_{\bar{\Omega}^{n}}=n \cdot \mathrm{Id}$. With the above notation, formula (2.1.3) may be rewritten in the form [GS]

$$
\left.\mathbf{i}\right|_{\bar{\Omega}^{n}}=\left.\left(\mathrm{Id}+\kappa+\cdots+\kappa^{n-1}\right) \mathrm{b}\right|_{\bar{\Omega}^{n} A}=\left.\mathrm{b} \mathrm{N} P\right|_{\bar{\Omega}^{n}} .
$$

Finally, following [CQ2], one defines the Connes differential by

$$
\left.\mathrm{B}\right|_{\bar{\Omega}^{n}}:=\left.\left(\mathrm{Id}+\kappa+\cdots+\kappa^{n}\right) \mathrm{d}\right|_{\bar{\Omega}^{n}}=\left.\mathrm{Nd} P\right|_{\bar{\Omega}^{n}} .
$$

The above formulas show that the operators $\mathbf{i}$ and $\mathrm{B}$ respect harmonic decomposition and one has

$$
\text { i d }=\mathrm{bNd} P=\mathrm{bB}, \quad \text { and } \quad \mathrm{d} \mathbf{i}=\mathrm{dbN} P=\mathrm{Ndb} P=\mathrm{Bb} .
$$

Thus, the equations $d \mathbf{i}+\mathbf{i} d=0$ and $B b+b B=0$ are equivalent.

The next lemma collects various technical properties of harmonic decomposition which will be used in various proofs (but not in the statements) in subsequent sections. 
Lemma 2.2.5. One has

$$
\begin{gathered}
P \overline{[\Omega, \Omega]}=P \mathrm{~b} \bar{\Omega}=\mathbf{i} \bar{\Omega} \\
P^{\perp} \overline{[\Omega, \Omega]}=P^{\perp} \bar{\Omega} \\
P^{\perp} \mathrm{d} \bar{\Omega}=(\mathrm{Id}-\kappa) \mathrm{d} \bar{\Omega}=\overline{[\mathrm{d} \Omega, \mathrm{d} \Omega]} \\
P^{\perp} \mathrm{b} \bar{\Omega}=(\mathrm{Id}-\kappa) \mathrm{b} \bar{\Omega} .
\end{gathered}
$$

Proof. Given a $\kappa$-action on a vector space $V$, let $V^{\kappa}=\left.\operatorname{ker}(\operatorname{Id}-\kappa)\right|_{V}$ denote the spaces of $\kappa$-invariants. If the $\kappa$-action on $V$ has finite order then $V=V^{\kappa} \oplus(\operatorname{Id}-\kappa) V$; in particular, the operator $(\operatorname{Id}-\kappa)^{2}$ acts on $V$ by zero if and only if $V=V^{\kappa}$.

It was observed in [CQ2] that $\kappa$ has finite order on $\mathrm{d} \bar{\Omega}$ and $\bar{\Omega} / \mathrm{b} \bar{\Omega}$. It follows that $\kappa$ has finite order also on $\bar{\Omega} / \mathrm{d} \bar{\Omega} \cong \mathrm{d} \bar{\Omega}$ and on $\mathrm{b} \bar{\Omega}=\mathrm{b}(\bar{\Omega} / \mathrm{b} \bar{\Omega})$. Each of these spaces is $\kappa$-stable. The first equation in (2.2.8) and equation (2.2.9) follow. Similarly, we deduce that the operator Id $-\kappa$ annihilates $P \bar{\Omega} / P$ b $\bar{\Omega}$, i.e., $(\operatorname{Id}-\kappa) P \bar{\Omega} \subset$ b $P \bar{\Omega}$.

Formula (2.2.1) readily implies the second equation in (2.2.8); it shows also that $(\operatorname{Id}-\kappa) \bar{\Omega}=$ $\overline{[\mathrm{d} A, \Omega]}$. Similarly, from formula (2.1.2) we get that $\mathrm{b} \bar{\Omega}=\overline{[A, \Omega]}$. Thus we obtain (cf. [CQ2]):

$$
\overline{[\Omega, \Omega]}=\overline{[A, \Omega]}+\overline{[\mathrm{d} A, \Omega]}=\mathrm{b} \bar{\Omega}+(\operatorname{Id}-\kappa) \bar{\Omega} .
$$

Applying $P$ to this equation and using the inclusion $(\mathrm{Id}-\kappa) P \bar{\Omega} \subset \mathrm{b} P \bar{\Omega}$ proved above, we deduce the first equation in (2.2.6). The second equation in (2.2.6) is clear from (2.2.3).

Finally, since $\operatorname{Id}-\kappa$ is invertible on $P^{\perp} \bar{\Omega}$, formula (2.2.10) shows that $P^{\perp} \bar{\Omega} \subseteq(\operatorname{Id}-\kappa) \bar{\Omega} \subseteq \overline{[\Omega, \Omega]}$. This yields the inclusion $P^{\perp} \bar{\Omega} \subseteq P^{\perp} \overline{[\Omega, \Omega]}$. The opposite inclusion is obvious, proving (2.2.7).

\section{MAIN CONSTRUCTIONS}

3.1. Construction of Hochschild and cyclic homology. Let $\bar{\Omega} A((u))$ be the space of formal Laurent series with coefficients in $\bar{\Omega} A$. We assign the variable $u$ degree -2 , so that the total degree of an element $f \in \bar{\Omega}^{p} A \cdot u^{-r}$ equals $|f|=p+2 r$. Thus, each of the differentials $u \mathrm{~B}$ and $\mathrm{b}$ has degree -1 .

Standard constructions of various versions of cyclic homology involve the $\mathbf{k}[u]$-modules $\mathbf{k} \llbracket u \rrbracket \subset$ $\mathbf{k}((u))$ and $R:=\mathbf{k}((u)) / u \mathbf{k} \llbracket u \rrbracket$. Specifically, reduced relative (to $\mathbf{k})$ Hochschild and cyclic homology of $A$ are defined as follows

$$
\begin{aligned}
\overline{\mathrm{HH}} \cdot(A) & =H(\bar{\Omega} A, \mathrm{~b}), & \overline{\mathrm{HC}} \cdot(A) & =H(\bar{\Omega} A \otimes R, \mathrm{~b}-u \mathrm{~B}), \\
\overline{\mathrm{HC}}_{\bullet}^{\mathrm{per}}(A) & =H(\bar{\Omega} A((u)), \mathrm{b}-u \mathrm{~B}), & \overline{\mathrm{HC}}_{\bullet}^{-}(A) & =H(\bar{\Omega} A \llbracket u \rrbracket, \mathrm{b}-u \mathrm{~B}) .
\end{aligned}
$$

A central result of this article is

Theorem 3.1.3. For a k-algebra A satisfying our standing assumptions, one has canonical isomorphisms:

$$
\begin{aligned}
& \mathrm{HH} .(A) \stackrel{\sim}{\rightarrow} \operatorname{ker}\left(\mathrm{i}: \overline{\mathrm{DR}}^{\bullet} \rightarrow \bar{\Omega}^{\bullet-1}\right) . \\
& \mathrm{HC} .(A) \stackrel{\sim}{\rightarrow} \operatorname{ker}\left(\mathrm{i}: \overline{\mathrm{DR}} \cdot \mathrm{d} \overline{\mathrm{DR}}^{\cdot-1} \rightarrow \bar{\Omega}^{-1} / \mathrm{d} \bar{\Omega}^{\bullet-2}\right) .
\end{aligned}
$$

Here, the first isomorphism is induced by the map $\bar{\Omega} \rightarrow \overline{\mathrm{DR}}, f \mapsto f_{\mathfrak{\natural}}$ and the second isomorphism is induced by the map $\bar{\Omega} \otimes R \rightarrow \overline{\mathrm{DR}}, \sum_{k \geq 0} f_{k} u^{-k} \mapsto\left(f_{0}\right)_{\text {t. }}$.

Isomorphism (3.1.4) has been already established in [GS, Theorem 4.1.1]. However, we will reproduce the proof, since most of the intermediate steps will also be used in the proof of (3.1.5).

Proof of Theorem 3.1 .3 First of all, from harmonic decomposition for $\bar{\Omega}$ and $\overline{[\Omega, \Omega]}$, respectively, using equation (2.2.7), we see that the projection $\bar{\Omega} \rightarrow P \bar{\Omega}$ induces an isomorphism $\bar{\Omega} / \overline{[\Omega, \Omega]} \stackrel{\sim}{\rightarrow}$ 
$P \bar{\Omega} / P \overline{[\Omega, \Omega]}$. Further, equation (2.2.6) yields $P \bar{\Omega} / \mathrm{b} P \bar{\Omega}=P \bar{\Omega} / P \overline{[\Omega, \Omega]}$. Also, the map i annihilates the space $P^{\perp} \bar{\Omega}$, by (2.2.3). Thus, we obtain a commutative diagram

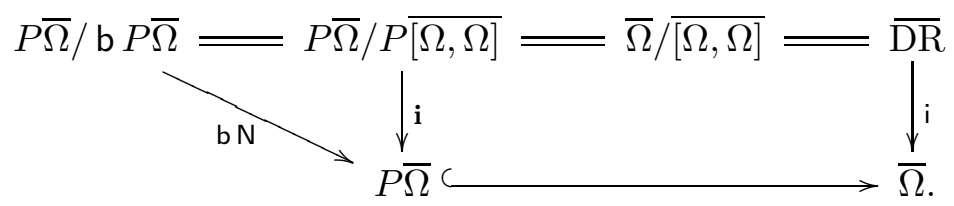

We see from the diagram that the assignment $f \mapsto f_{\natural}$ maps the space $H(P \bar{\Omega}, \mathrm{b})=\operatorname{ker}(\mathrm{b}$ : $P \bar{\Omega} / \mathrm{b} P \bar{\Omega} \rightarrow P \bar{\Omega})$ isomorphically onto the space $\operatorname{ker}(\mathrm{i}: \overline{\mathrm{DR}} \rightarrow \bar{\Omega})$.

Next, since $(\bar{\Omega}, \mathrm{d})$ is acyclic, it follows that its harmonic and anti-harmonic parts, $(P \bar{\Omega}, \mathrm{d})$ and $\left(P^{\perp} \bar{\Omega}, \mathrm{d}\right)$, are acyclic as well. Hence also $(P \bar{\Omega}, \mathrm{B})$ is acyclic, by (2.2.4). Cuntz and Quillen observed in [CQ2] that this implies that the cohomology of all of the complexes appearing in (3.1.1)-(3.1.2) are not affected by the replacement of the space $\bar{\Omega}$ by its harmonic part $P \bar{\Omega}$. Specifically, it follows from the above, using equations (2.2.2) and (2.2.4), that the following complexes are acyclic:

$$
\left(P^{\perp} \bar{\Omega}, \mathrm{b}\right), \quad\left(P^{\perp} \bar{\Omega}((u)), \mathrm{b}-u \mathrm{~B}\right), \quad\left(P^{\perp} \bar{\Omega} \llbracket u \rrbracket, \mathrm{b}-u \mathrm{~B}\right), \quad\left(P^{\perp} \bar{\Omega} \otimes R, \mathrm{~b}-u \mathrm{~B}\right) .
$$

Thus, the assignment $f \mapsto f_{\natural}$ gives an isomorphism $H\left(P^{\perp} \bar{\Omega}, \mathrm{b}\right) \stackrel{\sim}{\rightarrow} 0$, and (3.1.4) follows.

To proceed further, we need the following result of Cuntz and Quillen, [CQ2, Proposition 3.1].

Lemma 3.1.7. The projection modulo $u^{-1}$ and $\mathrm{im}(\mathrm{B})$ induces an isomorphism

$$
\overline{\mathrm{HC}} \cdot(A)=H \cdot(\bar{\Omega} \otimes R, \mathrm{~b}-u \mathrm{~B}) \stackrel{\sim}{\rightarrow} H \cdot(\bar{\Omega} / \mathrm{B} \bar{\Omega}, \mathrm{b}), \quad \sum_{k \geq 0} f_{k} \cdot u^{-k} \mapsto f_{0} \bmod \mathrm{B} \bar{\Omega} .
$$

Proof. Introduce an increasing filtration on $\bar{\Omega} \otimes R$ as follows:

$$
F_{j}(P \bar{\Omega} \otimes R):=P \bar{\Omega} \otimes\left(u^{-j} \mathbf{k} \llbracket u \rrbracket / u \mathbf{k} \llbracket u \rrbracket\right)=P \bar{\Omega} \otimes \operatorname{Span}\left(u^{-j}, u^{-j+1}, \ldots, u^{-1}, 1\right) .
$$

The fact that the complex $(P \bar{\Omega} \otimes R, \mathrm{~B})$ is acyclic implies that the standard spectral sequence associated to our filtration collapses at the second page to $H(P \bar{\Omega} / \mathrm{B} P \bar{\Omega}, \mathrm{b})$. We conclude that the map $\sum_{k \geq 0} f_{k} \cdot u^{-k} \mapsto f_{0}$ yields an isomorphism $H(P \bar{\Omega} \otimes R, \mathrm{~b}-u \mathrm{~B}) \stackrel{\sim}{\rightarrow} H(P \bar{\Omega} / \mathrm{B} P \bar{\Omega}, \mathrm{b})$, of harmonic components. The same map also gives an isomorphism $H\left(P^{\perp} \bar{\Omega} \otimes R, \mathrm{~b}-u \mathrm{~B}\right) \stackrel{\sim}{\rightarrow} H\left(P^{\perp} \bar{\Omega} / \mathrm{B} P^{\perp} \bar{\Omega}, \mathrm{b}\right)$, of anti-harmonic components. Since $\mathrm{B}\left(P^{\perp} \bar{\Omega}\right)=0$, the second cohomology group is $H\left(P^{\perp} \bar{\Omega}, \mathrm{b}\right)=$ 0 , so these cohomology groups vanish.

The isomorphism in (3.1.5) is now obtained as a composition:

$$
\begin{aligned}
\overline{\mathrm{HC}}(A) & =H(P \bar{\Omega} / \mathrm{B} P \bar{\Omega}, \mathrm{b}) \quad \text { by the proof of Lemma 3.1.7 } \\
& =\frac{\{f \in P \bar{\Omega} \mid \mathrm{b} f \in \mathrm{B} P \bar{\Omega}\}}{\mathrm{b} P \bar{\Omega}+\mathrm{B} P \bar{\Omega}} \\
& =\operatorname{ker}(\mathrm{b}: P \bar{\Omega} /(\mathrm{b} P \bar{\Omega}+\mathrm{B} P \bar{\Omega}) \rightarrow P \bar{\Omega} / \mathrm{B} P \bar{\Omega}) \\
& =\operatorname{ker}(\mathrm{i}:(P \bar{\Omega} / \mathrm{i} P \bar{\Omega}) / \mathrm{d}(P \bar{\Omega} / \mathrm{i} P \bar{\Omega}) \rightarrow P \bar{\Omega} / \mathrm{d} P \bar{\Omega}) \quad \text { by (2.2.3) }-(2.2 .4) \\
& =\operatorname{ker}(\mathrm{i}: \overline{\mathrm{DR}} / \mathrm{d} \overline{\mathrm{DR}} \rightarrow \bar{\Omega} / \mathrm{d} \bar{\Omega}), \quad \text { by (3.1.6). }
\end{aligned}
$$

3.2. Negative and periodic cyclic homology. Following [GS], we consider the bicomplex $(\bar{\Omega}((u))$, $u \mathrm{~d}$, $\mathbf{i}$ ), where each of the differentials $u \mathrm{~d}$ and $\mathbf{i}$ has degree -1 . The result below provides an interpretation of periodic, negative, and ordinary cyclic homology in terms of the noncommutative de Rham complex (in the latter case, this differs from the description of the previous section).

Note that the space $\overline{[d \Omega, d \Omega]} \subseteq \bar{\Omega}$ is annihilated by each of the differentials $d$ and $\mathbf{i}$. We view it also as a subspace of $\bar{\Omega} \llbracket u \rrbracket$ (and $\bar{\Omega}((u)))$, as constant terms in $u$. 
Theorem 3.2.1. There are natural isomorphisms

$$
\begin{aligned}
\overline{\mathrm{HC}}_{\cdot}^{\mathrm{per}}(A) & \cong H_{\cdot}(\bar{\Omega}((u)), \mathbf{i}-u \mathrm{~d}) \\
\overline{\mathrm{HC}}_{\cdot}^{-}(A) & \cong H \cdot(\bar{\Omega} \llbracket u \rrbracket, \mathbf{i}-u \mathrm{~d}) / \overline{[\mathrm{d} \Omega, \mathrm{d} \Omega]} \\
\overline{\mathrm{HC}} \cdot(A) & \cong H \cdot(\bar{\Omega} \otimes R, \mathbf{i}-u \mathrm{~d}) / \overline{[\mathrm{d} \Omega, \mathrm{d} \Omega]} .
\end{aligned}
$$

In the case of $(\overline{3.2 .3})$, the $\overline{[\mathrm{d} \Omega, \mathrm{d} \Omega]}$ includes into $H \cdot(\bar{\Omega} \llbracket u \rrbracket, \mathbf{i}-u \mathrm{~d})$ via the aforementioned inclusion into $\bar{\Omega} \llbracket u$ as constant terms in $u$. As we will see in the proof of theorem, the target coincides with the antiharmonic part of $H_{\bullet}(\bar{\Omega} \llbracket u \rrbracket, \mathbf{i}-u \mathrm{~d})$, and hence the inclusion is canonically split.

In (3.2.4), the inclusion of $\overline{[\mathrm{d} \Omega, \mathrm{d} \Omega]}$ into $H \cdot(\bar{\Omega} \otimes R, \mathbf{i}-u \mathrm{~d})$ is more complicated, but can also be given explicitly (in the proof of the theorem, we will see that the target is the antiharmonic part, so this inclusion is also canonically split). The inclusion in question is defined as follows.

Observe first that the map $\overline{\mathrm{d}}: \bar{\Omega} / \mathrm{d} \bar{\Omega} \rightarrow \mathrm{d} \bar{\Omega}$, induced by $\mathrm{d}$, is an isomorphism since the complex $(\bar{\Omega}, \mathrm{d})$ is acyclic. Write $\overline{\mathrm{d}}^{-1}: \mathrm{d} \bar{\Omega} \stackrel{\sim}{\rightarrow} \bar{\Omega} / \mathrm{d} \bar{\Omega}$ for an inverse isomorphism. Further, let $\tilde{\mathrm{d}}^{-1}: \mathrm{d} \bar{\Omega} \rightarrow \bar{\Omega}$ be a lift of $\overline{\mathrm{d}}^{-1}$. In other words, we fix an arbitrary (set-theoretic) section, $\tilde{\mathrm{d}}^{-1}$, of the surjection $\mathrm{d}: \bar{\Omega} \rightarrow \mathrm{d} \bar{\Omega}$.

With the above notation, the inclusion used in (3.2.4) is given by

$$
\overline{[\mathrm{d} \Omega, \mathrm{d} \Omega]} \hookrightarrow H_{\bullet}(\bar{\Omega} \otimes R, \mathbf{i}-u \mathrm{~d}), \quad f \mapsto\left(\mathrm{Id}-u^{-1} \tilde{\mathrm{d}}^{-1} \mathbf{i}\right)^{-1} \tilde{\mathrm{d}}^{-1} f .
$$

One can show that this formula makes sense and the result is independent of the choice of a section $\tilde{\mathrm{d}}^{-1}$, in a manner similar to the proof of Proposition 5.7.1 below.

In discussing Connes sequences involving these groups below, we will not use (3.2.4) but rather the description of (3.1.5). Isomorphism (3.2.2) was already proved in [GS, Theorem 4.2.2]; we included it in the above theorem for completeness only. For a related discussion of negative cyclic homology see also [GS].

Proof of (3.2.3) and (3.2.4). We first deal with (3.2.3). Similarly to the arguments in the proof of Theorem 3.1.3,

$$
\overline{\mathrm{HC}}^{-}(A)=H(\bar{\Omega} \llbracket u \rrbracket, \mathrm{b}-u \mathrm{~B})=H(P \bar{\Omega} \llbracket u \rrbracket, \mathrm{b}-u \mathrm{~B})=H(P \bar{\Omega} \llbracket u \rrbracket, \mathbf{i}-u \mathrm{~d}) .
$$

Now, thanks to equation (2.2.8), we have embeddings $\overline{[\mathrm{d} \Omega, \mathrm{d} \Omega]} \stackrel{\sim}{\rightarrow} P^{\perp} \mathrm{d} \bar{\Omega} \hookrightarrow P^{\perp} \bar{\Omega} \hookrightarrow P^{\perp} \bar{\Omega} \llbracket u \rrbracket$. To complete the proof, it suffices to show that the composite embedding yields a quasi-isomorphism of complexes

$$
\overline{[\mathrm{d} \Omega, \mathrm{d} \Omega]} \rightarrow\left(P^{\perp} \bar{\Omega} \llbracket u \rrbracket, \mathbf{i}-u \mathrm{~d}\right),
$$

where the LHS is considered as a complex with zero differential.

To this end, we observe that the operator $\mathbf{i}$ vanishes on $P^{\perp} \bar{\Omega}$ and the complex $(\bar{\Omega}, \mathrm{d})$ is acyclic. Therefore, we find

$$
H \cdot\left(P^{\perp} \bar{\Omega} \llbracket u \rrbracket, \mathbf{i}-u \mathrm{~d}\right)=H_{\cdot}\left(P^{\perp} \bar{\Omega} \llbracket u \rrbracket,-u \mathrm{~d}\right) \cong \mathrm{d} P^{\perp} \bar{\Omega}^{\bullet} .
$$

The term on the right equals $\overline{[d \Omega, d \Omega]}$ by $(2.2 .8)$, proving that $(\underline{3.2 .5})$ is a quasi-isomorphism.

For the isomorphism (3.2.4), we can apply the same argument as above to conclude that

$$
\overline{\mathrm{HC}}(A)=H(P \bar{\Omega} \otimes R, \mathrm{~b}-u \mathrm{~B})=H(P \bar{\Omega} \otimes R, \mathbf{i}-u \mathrm{~d}) .
$$

Then, since $\mathbf{i}$ vanishes on $P^{\perp} \bar{\Omega}$ and the complex $\left(P^{\perp} \bar{\Omega}, \mathrm{d}\right)$ is acyclic, we obtain

$$
H\left(P^{\perp} \bar{\Omega} \otimes R, \mathrm{~d}\right)=P^{\perp} \bar{\Omega} / u \mathrm{~d}\left(u^{-1} P^{\perp} \bar{\Omega}\right) \cong P^{\perp} \bar{\Omega} / \mathrm{d} P^{\perp} \bar{\Omega} \stackrel{\overline{\mathrm{d}}}{\rightarrow} \mathrm{d} P^{\perp} \bar{\Omega} \cong \overline{[\mathrm{d} \Omega, \mathrm{d} \Omega]} .
$$

Finally, note that the resulting isomorphism $H\left(P^{\perp} \bar{\Omega} \otimes R, \mathrm{~d}\right) \cong \overline{[\mathrm{d} \Omega, \mathrm{d} \Omega]}$ is indeed inverted by the claimed formula $\left(\mathrm{Id}-u^{-1} \tilde{\mathrm{d}}^{-1} \mathbf{i}\right)^{-1} \tilde{\mathrm{d}}^{-1}$, provided the chosen section $\tilde{\mathrm{d}}^{-1}$ respects harmonic decomposition (or at least preserves antiharmonic forms). In fact, in this case, since $\mathbf{i}$ is zero on 
antiharmonic forms, the resulting map reduces to $\tilde{\mathrm{d}}^{-1}=P^{\perp} \tilde{\mathrm{d}}^{-1}$. On the other hand, as observed above, the formula does not actually depend on the choice of $\tilde{d}^{-1}$.

\section{THE CONNES EXACT SEQUENCE}

4.1. Connes exact sequence via noncommutative differential forms. We now construct a version of Connes' exact sequence, involving reduced homology groups, using the interpretation for cyclic and Hochschild homology provided by Theorem 3.1.3.

Theorem 4.1.1. There is an exact sequence,

$$
0 \rightarrow \overline{\mathrm{HD}}_{n}(A) \stackrel{S_{2}}{\longrightarrow} \overline{\mathrm{HC}}_{n}(A) \stackrel{B}{\longrightarrow} \overline{\mathrm{HH}}_{n+1}(A) \stackrel{I}{\longrightarrow} \overline{\mathrm{HC}}_{n+1}(A) \stackrel{S_{1}}{\longrightarrow} \overline{\mathrm{HD}}_{n-1}(A) \rightarrow 0,
$$

where the maps $S_{2}, B, I$, and $S_{1}$ are defined by the following assignments:

$$
S_{2} f_{\natural}=f_{\natural}, \quad B f_{\natural}=(\mathrm{d} f)_{\natural}, \quad I f_{\natural}=f_{\natural}, \quad S_{1}\left(f_{\natural}\right)=\left(\overline{\mathrm{d}}^{-1} \mathrm{i} f_{\natural}\right)_{\natural} .
$$

Note here that the map $B: \overline{\mathrm{HC}}_{n}(A) \rightarrow \overline{\mathrm{HH}}_{n-1}(A)$ is distinguished by its font from the differential B on $\Omega$ and $\bar{\Omega}$. We let $S:=S_{2} \circ S_{1}: \overline{\mathrm{HC}}_{n}(A) \rightarrow \overline{\mathrm{HC}}_{n-2}(A)$. This is the standard periodicity map which splices the above exact sequence into the more familiar Connes' long exact sequence. The exact sequence of Theorem 4.1.1 incorporates additional information, implying that $\overline{\mathrm{HD}} .(A)=\operatorname{ker}(B)$ (the Connes-Karoubi theorem).

We now prove that the above maps are well-defined. We will prove that they coincide with the usual definitions in 4.2 , and give a direct proof of exactness (using the right hand sides of (3.1.4)-(3.1.5) as definitions) in $\$ 4.3$.

The map $S_{2}$ is obtained from the clearly well-defined map $\operatorname{ker}\left(\left.\mathrm{d}\right|_{\overline{\mathrm{DR}}}\right) / \mathrm{d} \overline{\mathrm{DR}} \rightarrow \overline{\mathrm{DR}} / \mathrm{d} \overline{\mathrm{DR}}$; we need to check that the image is contained in the kernel of $i$. Indeed, if $(\mathrm{d} f)_{\natural}=0$, then $\mathrm{di} f_{\natural}=$ $-\mathrm{i}(\mathrm{d} f)_{\natural}=0$, and hence $\mathrm{i} f_{\natural} \in \mathrm{d} \bar{\Omega}$, as required.

We now show that $B$ is well-defined. First note that, if $f_{\natural} \in \overline{\mathrm{DR}} / \mathrm{d} \overline{\mathrm{DR}}$, then $(\mathrm{d} f)_{\natural} \in \overline{\mathrm{DR}}$ makes sense independently of the choice of representative element $f \in \bar{\Omega}$. Next, if i $f_{\natural} \in \mathrm{d} \bar{\Omega}$, then $\mathrm{i}(\mathrm{d} f)_{\natural}=$ - d i $f_{\natural}=0$, so $(\mathrm{d} f)_{\natural} \in \overline{\mathrm{DR}}$ defines a class in Hochschild homology.

It is immediate that $I$ is well-defined.

To prove that $S_{1}$ is well defined, we compute

$$
\overline{\mathrm{d}}^{-1} \mathrm{id} \overline{\mathrm{DR}}=\overline{\mathrm{d}}^{-1} \mathrm{di} \overline{\mathrm{DR}}=(\mathrm{i} \overline{\mathrm{DR}}+\mathrm{d} \bar{\Omega}) / \mathrm{d} \bar{\Omega} .
$$

Since i $\overline{\mathrm{DR}} \subseteq \overline{[A, \Omega]}$, by $(2.1 .3)$, the right most term above projects to zero in $\overline{\mathrm{DR}} / \mathrm{d} \overline{\mathrm{DR}}$. It follows that the formula for $S_{1}$ gives a well defined map $S_{1}: \overline{\mathrm{HC}}(A) \rightarrow \overline{\mathrm{DR}} / \mathrm{d} \overline{\mathrm{DR}}$. It remains to check that $\mathrm{d} S_{1}(f)=0$ for every $f \in \overline{\mathrm{DR}}$ such that i $f \in \mathrm{d} \bar{\Omega}$. Indeed, $\mathrm{d} S_{1}(f)=\mathrm{d}\left(\overline{\mathrm{d}}^{-1} \text { if }\right)_{\natural}=(\mathrm{i} f)_{\natural}=0$, since i $\overline{\mathrm{DR}} \subseteq \overline{[A, \Omega]}$.

4.2. Comparison of maps in (4.1.2) with the usual definitions. We now show that the maps in (4.1.2) coincide with the usual ones up to nonzero integer multiples depending on degree (i.e., a nonvanishing expression involving the aforementioned degree operator $\mathrm{N}$ ). We will make use of the fact that every homology class can be represented by a harmonic cycle.

Here and later on we will make use of the notation $f_{0}$ for the constant term in $u$ of an element $f \in \bar{\Omega}((u))$ (or $f \in \bar{\Omega} \llbracket u \rrbracket)$.

4.2.1. The map $B$. If $f$ is a harmonic cycle with respect to the usual definition of Hochschild or cyclic homology, then (2.2.3) implies that $\mathrm{B} f=\mathrm{Nd} f$. Since the usual $B$ is essentially defined by applying $B$ and our version is defined by applying $d$, this shows that the difference is postcomposition with $\mathrm{N}$, which is nonvanishing since $\mathrm{d}$ has de Rham degree 1. 
4.2.2. The map $I$. The map $I$ is defined by leaving the harmonic cycle representing a homology class unchanged, and mapping it from one complex to another (throwing out the coefficients of $u^{>0}$ in the case of $I$ acting on the usual definition of cyclic homology). Since the isomorphisms from ordinary versions to our versions of Hochschild and cyclic homology also leave the harmonic cycles unchanged, we obtain the desired compatibility.

4.2.3. The maps $S_{1}$ and $S_{2}$. Let us consider $S_{1}$. Suppose that $f \in \bar{\Omega}((u)) / u \bar{\Omega} \llbracket u \rrbracket$ is a harmonic cycle of degree $n+1$, i.e., $|f|=n+1$ under the total degree in which $\left|\bar{\Omega}^{j}\right|=j$ and $|u|=-2$. The usual periodicity operation is $f \mapsto u \cdot f$. We need to show that $\mathrm{i}\left(f_{0}\right)_{\natural}=\mathrm{d}\left((u f)_{0}\right)_{\natural}$. Since $(\mathbf{i}-u \mathrm{~d}) f=0$, this is immediate.

Regarding the map $S_{2}$, we only need to observe that the usual formula for $S_{2}$ is uniquely determined by the fact that the part mapping to a coefficient of $u^{0}$ is the obvious map (leaving harmonic cycles unchanged). (To obtain the coefficients of $u^{<0}$, we can use the usual periodicity map $S=S_{2} \circ S_{1}$, which is multiplication by $u$. Then, our construction yields the explicit formula $\overline{\mathrm{d}}^{-1} \mathrm{i}$ for this operation, by the previous paragraph.)

4.3. Direct proof of exactness. Since we showed that the maps in (4.1.2) are compatible with the isomorphisms between the new versions of cyclic and Hochschild homology and the usual ones, exactness follows from the usual construction of (4.1.2) by a commutative diagram of short exact sequences of the complexes computing Hochschild and cyclic homologies (cf. [Lo]).

Nonetheless, here we will prove exactness directly using our formulas (3.1.4)-(3.1.5).

Injectivity of $S_{2}$ : By definition, $\overline{\mathrm{HD}}(A) \subseteq \overline{\mathrm{DR}} / \mathrm{d} \overline{\mathrm{DR}}$.

$\overline{\operatorname{im}\left(S_{2}\right)=\operatorname{ker}(B)}$ : By definition, the cycles in $\operatorname{ker}(B)$ are represented by $f_{\natural} \in \overline{\mathrm{DR}}^{n}$ such that $\mathrm{d} f_{\natural}=0$ and $\mathrm{i} f_{\natural} \in \mathrm{d} \bar{\Omega}$. The second condition is equivalent to $\mathrm{d}$ i $f_{\natural}=0$, which follows from the first condition. We deduce that $\operatorname{ker}(B)=\overline{\mathrm{HD}}_{n}(A)$, as desired.

$\underline{\operatorname{im}}(B)=\operatorname{ker}(I)$ : The cycles in $\operatorname{ker}(I)$ are those elements $f_{\natural} \in \overline{\mathrm{DR}}^{n}$ such that $\mathrm{i} f_{\natural}=0$ and $f_{\natural} \in$ $\mathrm{d} \overline{\mathrm{DR}}^{n-1}$. Given the second condition, we can assume $f=\mathrm{d} g$. Then $\mathrm{i}(\mathrm{d} g)_{\natural}=\mathrm{id} g_{\natural}=0$ holds if and only if $\mathrm{i} g_{\natural} \in \mathrm{d} \bar{\Omega}$, i.e., $g_{\natural} \in \overline{\mathrm{HC}}_{n-1}(A)$. Conversely, it is clear that $\operatorname{im}(B) \subseteq \operatorname{ker}(I)$. We conclude that $\operatorname{ker}(I)=\operatorname{im}(B)$.

$\operatorname{im}(I)=\operatorname{ker}\left(S_{1}\right)$ : Every cycle in $\operatorname{ker}\left(S_{1}\right)$ is represented by a harmonic element $f_{\natural} \in \overline{\mathrm{DR}}$ such that i $f_{\natural} \overline{\mathrm{d}} \overline{[\Omega, \Omega]}$. By (2.2.6),$P \overline{[\Omega, \Omega]}=\mathbf{i} \bar{\Omega}$. We deduce that

$$
\mathrm{i} f_{\natural}=P \mathrm{i} f_{\natural} \in P \mathrm{~d} \overline{[\Omega, \Omega]}=\mathrm{d} P \overline{[\Omega, \Omega]}=\mathrm{d} \mathbf{i} \bar{\Omega}=\mathrm{i} \mathrm{d} \bar{\Omega} \text {. }
$$

It follows that one can find $g, h \in \bar{\Omega}$ such that $\mathrm{i}\left(g_{\natural}\right)=0$ and $f_{\natural}=g_{\natural}+\mathrm{d} h_{\natural}$. Thus, in $\overline{\mathrm{HC}}(A)$, we obtain $f_{\natural} \bmod \mathrm{d} \overline{\mathrm{DR}}=I\left(g_{\natural}\right)$, as desired.

Surjectivity of $S_{1}$ : Every class in $\overline{\operatorname{HD}}(A)$ is represented by a harmonic element $f \in \bar{\Omega}$ such that

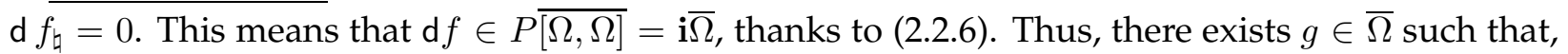
in $\overline{\mathrm{HD}}(A)$, we obtain $f_{\natural}=\overline{\mathrm{d}}^{-1} \mathbf{i}(g)=S_{1}\left(g_{\natural}\right)$, as desired.

4.4. Connes' exact sequences for periodic and negative cyclic homology. Now, using Theorem 3.2.1 (for periodic and negative cyclic homology) as well as Theorem 3.1 .3 (for Hochschild and ordinary cyclic homology), we construct versions of Connes' exact sequences involving periodic and negative cyclic homology.

It will be convenient below to introduce, for each $n=0,1, \ldots$, the following space of degree $n$ elements in $\bar{\Omega} \llbracket u \rrbracket$ :

$$
\begin{aligned}
\mathbf{Z}_{n}(A) & :=\left\{f \in(\bar{\Omega} \llbracket u \rrbracket)_{n} \mid(\mathbf{i}-u \mathrm{~d}) f \quad \text { is independent of } u\right\} \\
& =\left\{f=f^{n}+u f^{n+2}+u^{2} f^{n+4}+\left.\ldots\right|_{9} f^{j} \in \bar{\Omega}^{j}, \mathrm{~d} f^{j}=\mathbf{i}\left(f^{j+2}\right), \forall j=n, n+2, \ldots\right\} .
\end{aligned}
$$


We observe that, for every element $f=f^{n}+u f^{n+2}+u^{2} f^{n+4}+\ldots \in \mathrm{Z}_{n}(A)$, in $\overline{\mathrm{DR}}^{n+1}$, one has $\mathrm{d}\left(f^{n}\right)_{\natural}=\left(\mathbf{i}\left(f^{n+2}\right)\right)_{\natural}=0$. Therefore, the assignment sending $f=f^{n}+u f^{n+2}+u^{2} f^{n+4}+\ldots$ to $\left(f^{n}\right)_{\natural} \in \overline{\mathrm{DR}}^{n}$ gives a well defined map $I^{\prime}: \mathrm{Z}(A) \rightarrow\{\alpha \in \overline{\mathrm{DR}} \mid \mathrm{d} \alpha=0\}$. In particular, one has an induced map

$$
I_{\mathrm{HD}}^{\prime}: \mathrm{Z}(A) \rightarrow \overline{\mathrm{HD}}(A)=\{\alpha \in \overline{\mathrm{DR}} \mid \mathrm{d} \alpha=0\} / \mathrm{d} \overline{\mathrm{DR}} .
$$

Next, it is clear from the definition that, for $f=f^{n}+u f^{n+2}+u^{2} f^{n+4}+\ldots \in \mathrm{Z}_{n}(A)$, the condition $(\mathbf{i}-u \mathrm{~d}) f=0$ is equivalent to $\mathbf{i}\left(f^{n}\right)=0$. Also, since $\mathbf{i} \bar{\Omega}$ projects to zero in $\overline{\mathrm{DR}}$, the map $I^{\prime}$ sends the subspace $(\mathbf{i}-u \mathrm{~d}) \bar{\Omega} \llbracket u \rrbracket \subseteq \mathrm{Z}(A)$ of boundaries to zero. Hence, using (3.1.4), we deduce that the map $I^{\prime}$ gives a well defined morphism

$$
I_{\mathrm{HH}}^{\prime}: H_{.}(\bar{\Omega} \llbracket u \rrbracket, \mathbf{i}-u \mathrm{~d}) \rightarrow \overline{\mathrm{HH}} .(A) .
$$

We define

$$
\begin{aligned}
& \overline{\mathrm{HD}}^{\prime}(A):=\operatorname{im}\left[I_{\mathrm{HD}}^{\prime}: \mathrm{Z}(A) \rightarrow \overline{\mathrm{HD}}(A)\right] \\
& \overline{\mathrm{HH}}^{\prime}(A):=\operatorname{im}\left[I_{\mathrm{HH}}^{\prime}: H \cdot(\bar{\Omega} \llbracket u \rrbracket, \mathbf{i}-u \mathrm{~d}) \rightarrow \overline{\mathrm{HH}}(A)\right] .
\end{aligned}
$$

Theorem 4.4.3. There is a commutative diagram with exact rows,

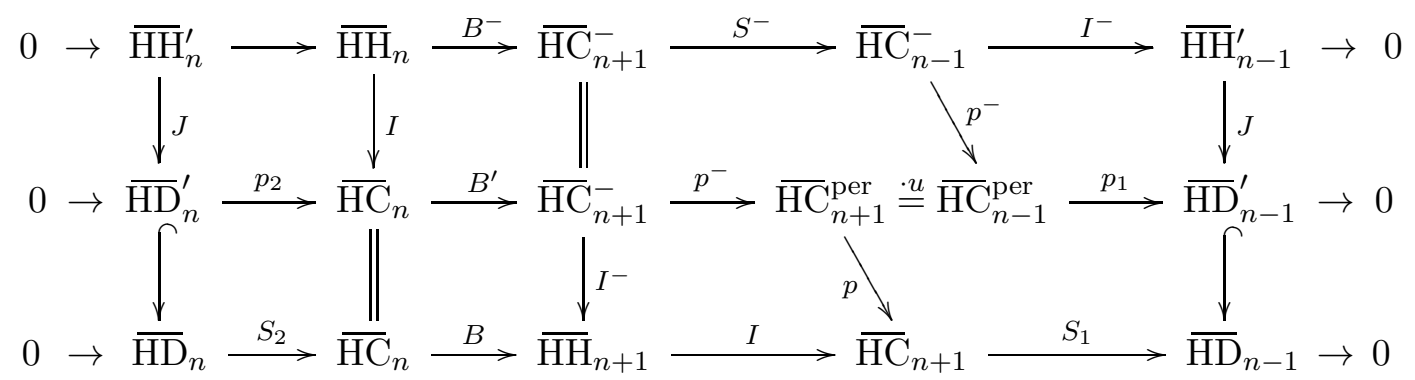

The maps in the diagram are defined by the assignments

$$
\begin{gathered}
p_{2} f_{\natural}=f_{\natural}, \quad B^{-}\left(f_{\natural}\right)=\mathrm{d} f, \quad B^{\prime}\left(f_{\natural}\right)=\mathrm{d} f, \quad S^{-}(f)=u f, \quad p^{-}(f)=f, \\
I^{-}(f)=\left(f_{0}\right)_{\natural}, \quad p_{1}(f)=\left(f_{0}\right)_{\natural}, \quad J f_{\natural}=f_{\natural}, \quad p=p_{2} \circ p_{1} .
\end{gathered}
$$

Let us explain why all the maps above are well-defined. For $B^{-}$, it is immediate that $B^{-}=B^{\prime} I$, so it is enough to show that $B^{\prime}$ is well-defined. To this end, let $f \in \bar{\Omega}$ satisfy i $f_{\natural} \in \mathrm{d} \bar{\Omega}$. Then, $(\mathbf{i}-u \mathrm{~d}) \mathrm{d} f=0$, so that $\mathrm{d} f$ indeed defines a class in negative cyclic homology. We then need to show that $\mathrm{d} \overline{[\Omega, \Omega]} \subseteq(\mathbf{i}-u \mathrm{~d}) \bar{\Omega} \llbracket u \rrbracket+\overline{[\mathrm{d} \Omega, \mathrm{d} \Omega]}$. To prove this, we write $\overline{[\Omega, \Omega]}=P \overline{[\Omega, \Omega]}+P^{\perp} \overline{[\Omega, \Omega]}$. We know that $P \overline{[\Omega, \Omega]}=\mathbf{i} \bar{\Omega}$, by (2.2.6). Further, we compute

$$
P^{\perp} \overline{[\Omega, \Omega]} \stackrel{[2.2 .10}{=} P^{\perp} \mathrm{b} \bar{\Omega}+P^{\perp}(\mathrm{Id}-\kappa) \bar{\Omega} \stackrel{\sqrt[2.2 .9]{=}}{=}(\operatorname{Id}-\kappa) \mathrm{b} \bar{\Omega}+(\mathrm{Id}-\kappa) P^{\perp} \bar{\Omega} \subset(\operatorname{Id}-\kappa) \bar{\Omega}=\overline{[\mathrm{d} A, \Omega]} .
$$

Thus, we have proved the inclusion we need: $\overline{[\Omega, \Omega]} \subset \mathbf{i} \bar{\Omega}+\overline{[\mathrm{d} \Omega, \Omega]}$.

It is immediate that $S^{-}$is well-defined. To show that $p^{-}$is well-defined, we only need to show that the space $\overline{[d \Omega, d}]]$ is contained in the image of the differential $\mathbf{i}-u \mathrm{~d}$, on $\bar{\Omega}((u))$. This follows from the fact that $\mathrm{i} \overline{[\Omega, d \Omega]}=0$.

For the map $I^{-}$, we observe that this map is induced by the map (4.4.2). This makes sense since the map $I^{\prime}$ annihilates the space $\overline{[\mathrm{d} \Omega, \mathrm{d} \Omega]} \subseteq \mathrm{Z}(A)$.

Finally, it is immediate that $p_{1}$ and $J$ are well-defined, and that the diagram commutes. 
4.5. Comparison of the maps in (4.4.4) with the usual definitions. The fact that $B^{-}$and $B^{\prime}$ coincide with the usual definitions is the same as the reason we gave for $B$ in 4.2 these maps take harmonic representatives $f$ to $\mathrm{d} f$ under our definitions, and to $\mathrm{B} f$ in the usual definitions, which differ by a scalar depending on degree. Similarly, for $I^{-}$and $J$, these maps leave harmonic representatives unchanged.

The fact that the periodicity map $S^{-}$is compatible with our isomorphism is obvious, since it is defined by multiplication by $u$ in both cases.

Finally, the maps $p$ and $p^{-}$, like the case of the maps of type $I$, send harmonic cycles to the classes represented by the same harmonic cycles (in the case of $p$, taking the coefficients of $u \leq 0$ or $u^{0}$ ).

\subsection{Direct proof of exactness in diagram 4.4.4).}

4.6.1. The middle row of (4.4.4). Injectivity of $p_{2}$ : This is clear from construction (or from injectivity of $\left.S_{2}\right)$.

$\operatorname{im}\left(p_{2}\right)=\operatorname{ker}\left(B^{\prime}\right)$ : The cycles in $\operatorname{ker}\left(B^{\prime}\right)$ are represented by $f_{\natural} \in \overline{\mathrm{HC}}(A)$ such that $\mathrm{d} f=(\mathbf{i}-u \mathrm{~d}) g+$

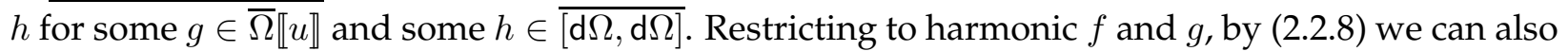
set $h=0$. Then, $(\mathbf{i}-u \mathrm{~d})(f+u g)=\mathrm{i} f_{\natural} \in \mathrm{d} \bar{\Omega}$. So $\operatorname{ker}\left(B^{\prime}\right) \subseteq \operatorname{im}\left(p_{2}\right)$. For the opposite inclusion, if $f \in \mathbf{Z}(A)$, then $(\mathbf{i}-u \mathrm{~d}) f=\mathrm{i}\left(f_{0}\right)_{\natural} \in \mathrm{d} \bar{\Omega}$. Thus, $\mathrm{d} f_{0}=(\mathbf{i}-u \mathrm{~d}) u^{-1}\left(f_{0}-f\right)$, so $\left(f_{0}\right)_{\natural} \in \operatorname{ker}\left(B^{\prime}\right)$.

$\operatorname{im}\left(B^{\prime}\right)=\operatorname{ker}\left(p^{-}\right)$: The elements in $\operatorname{ker}\left(p^{-}\right)$are represented by harmonic elements $f \in \bar{\Omega} \llbracket u \rrbracket$ such that $f=(\mathbf{i}-u \mathbf{d}) g$ where $g \in \bar{\Omega}((u))$ is harmonic. Replacing $f$ with $f-(\mathbf{i}-u \mathbf{d}) \sum_{j \geq 0} g_{j} u^{j}$, we can assume that $g_{j}=0$ for $j \geq 0$. Then, $f=f_{0}=\mathrm{d} g_{-1}$, and $\mathrm{i}\left(g_{-1}\right)_{\natural} \in \mathrm{d} \bar{\Omega}$. Hence, $\left(g_{-1}\right)_{\natural} \in \overline{\mathrm{HC}}(A)$ and $f=B^{\prime}\left(g_{-1}\right)_{\text {Ł. }}$. Therefore, $\operatorname{ker}\left(p^{-}\right) \subseteq \operatorname{im}\left(B^{\prime}\right)$. For the opposite inclusion, suppose that $f \in \operatorname{im}\left(B^{\prime}\right)$. That is, there exists $h \in \bar{\Omega}$ satisfying $f=\mathrm{d} h$ and $\mathrm{i} h_{\natural} \in \mathrm{d} \bar{\Omega}$. Now, we can set $g_{-1}:=h$, and inductively we can choose $g_{-j}$ for $j>1$ such that $\mathrm{d} g_{-j}=\mathbf{i} g_{1-j}$ for all $j>1$. We deduce that $-(\mathbf{i}-u \mathrm{~d}) g=\mathrm{d} h=f$. Therefore, $\operatorname{im}\left(B^{\prime}\right) \subseteq \operatorname{ker}\left(p^{-}\right)$.

$u \operatorname{im}\left(p^{-}\right)=\operatorname{ker}\left(p_{1}\right)$ : Elements of $\operatorname{ker}\left(p_{1}\right)$ are $f \in \bar{\Omega}((u))$ such that $f_{0} \in \mathrm{d} \bar{\Omega}+\overline{[\Omega, \Omega]}$ and $(\mathbf{i}-u \mathrm{~d}) f=$ 0. We claim first that $f_{j} \in \mathrm{d} \bar{\Omega}+\overline{[\Omega, \Omega]}$ for all $j \leq 0$. Indeed, $\mathrm{d} f_{j}=\mathrm{i}\left(f_{j+1}\right)_{\natural}$ for $j<0$. So, if $f_{j+1} \in \mathrm{d} \bar{\Omega}+\overline{[\Omega, \Omega]}$, then $\mathrm{d} f_{j}=\mathrm{i}\left(f_{j+1}\right)_{\natural} \in \mathbf{i} \mathrm{d} \bar{\Omega}=\mathrm{d} \mathbf{i} \bar{\Omega} \subseteq \mathrm{d} \overline{[\Omega, \Omega]}$. By acyclicity of $(\bar{\Omega}, \mathrm{d})$, we conclude that $f_{j} \in \mathrm{d} \bar{\Omega}+\overline{[\Omega, \Omega]}$ as well, so by induction, we conclude the desired result.

Suppose further that $f$ is harmonic. Then, $f_{0} \in \mathrm{d} P \bar{\Omega}+\mathbf{i} P \bar{\Omega}$ since $P \overline{[\Omega, \Omega]}=\mathbf{i} P \bar{\Omega}$, by (2.2.6). Writing $f_{0}=\mathrm{d} g+\mathrm{i} h_{\natural}$, we conclude that $f^{\prime}:=f+(\mathbf{i}-u \mathrm{~d})\left(u^{-1} g-h\right)$ satisfies $f_{0}^{\prime}=0$, and $f_{j}^{\prime} \in \mathrm{d} \bar{\Omega}+\overline{[\Omega, \Omega]}$ for $j<0$. Applying the same reasoning to $u f^{\prime}$, we eventually conclude that $f$ is homologous to a harmonic cycle $\tilde{f} \in u P \bar{\Omega} \llbracket u \rrbracket$. Therefore, $f \in u \cdot \operatorname{im}\left(p^{-}\right)$.

The converse containment, $u \cdot \operatorname{im}\left(p^{-}\right) \subseteq \operatorname{ker}\left(p_{1}\right)$, is obvious.

Surjectivity of $p_{1}$ : Suppose that $f \in \mathrm{Z}(A)$. Then $(\mathbf{i}-u \mathrm{~d}) f=\mathrm{i}\left(f_{0}\right)_{\natural} \in \mathrm{d} \bar{\Omega}$. As observed above, setting $g_{0}=f_{0}$, we can choose $g_{-j}$ for $j \geq 1$ such that $\mathrm{d} g_{-j}=\mathbf{i} g_{1-j}$ for all $j \geq 1$. Set $h:=g+\left(f-f_{0}\right)$. Then, $(\mathbf{i}-u \mathrm{~d}) h=-u \mathrm{~d} f_{0}-\mathrm{i}\left(f_{0}\right)_{\natural}+\left(u \mathrm{~d} f_{0}+\mathrm{i}\left(f_{0}\right)_{\natural}\right)=0$. Since $\left(h_{0}\right)_{\natural}=\left(f_{0}\right)_{\natural}$, we conclude that $\left(f_{0}\right)_{\natural}=p_{1}(h)$ is in the image of $p_{1}$.

4.6.2. The top row of (4.4.4). The first map is tautologically injective.

$\operatorname{ker}\left(B^{-}\right)=\overline{\mathrm{HH}}^{\prime}(A)$ : Assume that $f$ is harmonic and i $f_{\natural}=0$, and suppose that $f_{\natural} \in \overline{\mathrm{HH}}(A)$ satisfies $\mathrm{d} f=(\mathbf{i}-u \mathrm{~d}) g$ for some harmonic $g \in \bar{\Omega} \llbracket u \rrbracket$. Then, $(\mathbf{i}-u \mathrm{~d})(f-u g)=0$, so $f_{\natural} \in \overline{\mathrm{HH}}_{n}^{\prime}(A)$. Thus $\operatorname{ker}\left(B^{-}\right) \subseteq \overline{\mathrm{HH}}^{\prime}(A)$. Conversely, if $f \in \operatorname{ker}(\mathbf{i}-u \mathrm{~d}) \subseteq \bar{\Omega} \llbracket u \rrbracket$, then $\mathrm{d} f_{0}=(\mathbf{i}-u \mathrm{~d})\left(f-f_{0}\right)$, so $\left(f_{0}\right)_{\natural} \in \operatorname{ker}\left(B^{-}\right)$.

$\operatorname{im}\left(B^{-}\right)=\operatorname{ker}\left(S^{-}\right)$: The kernel of $S^{-}$is represented by elements of the form $u^{-1}(\mathbf{i}-u \mathrm{~d}) g$, where $g \overline{\in P \bar{\Omega} \llbracket u \rrbracket \text { satisfies } \mathrm{i}}\left(g_{0}\right)_{\mathfrak{\natural}}=0$. Up to coboundary, this is the same as elements $\mathrm{d} g_{0}$ such that 
$\mathrm{i}\left(g_{0}\right)_{\natural}=0$, i.e., the image of $B^{-}$. Conversely, if $\mathrm{i}\left(g_{0}\right)_{\natural}=0$, then $B^{-}\left(g_{0}\right)_{\natural}=\mathrm{d} g_{0}=-u^{-1}(\mathbf{i}-u \mathrm{~d})\left(g_{0}\right)$ is in the kernel of $S^{-}$.

$\operatorname{im}\left(S^{-}\right)=\operatorname{ker}\left(I^{-}\right)$: The kernel of $I^{-}$is represented by elements $f \in \bar{\Omega} \llbracket u \rrbracket$ such that $f_{0} \in \overline{[\Omega, \Omega]}$. If we assume that $f$ is harmonic, then $f_{0} \in \mathrm{b} P \bar{\Omega}=\mathbf{i} P \bar{\Omega}$, see (2.2.6). Write $f_{0}=\mathrm{i} g_{\natural}$ for some $g$. Then, $f-(\mathbf{i}-u \mathrm{~d}) g \in u \bar{\Omega} \llbracket u \rrbracket$. Hence, the class of $f$ is in the image of $S^{-}$. Conversely, it is clear that the image of $S^{-}$maps to zero under $I^{-}$.

Surjectivity of $I^{-}$: This is immediate from the definition of $\overline{\mathrm{HH}}^{\prime}(A)$.

\section{EXTENDED KAROUBI-DE RHAM COMPLEX AND CYCLIC HOMOLOGY}

In this section, we will consider, following [GS], an "extended" version, $\overline{\mathrm{DR}}_{t} A$, of the noncommutative de Rham complex. One of the reasons for introducing such an extended version is that it maps naturally to the equivariant (as opposed to the ordinary) de Rham complex of representation varieties of the algebra in question, see $₫ 8$.

Another important feature of $\overline{\mathrm{DR}}_{t}$ is that it comes equipped with natural anti-commuting differentials $\mathrm{d}$ and $\mathrm{i}_{t}$. We will show that the homology of the complex $\left(\overline{\mathrm{DR}}_{t} A \otimes R, \mathrm{i}_{t}-u \mathrm{~d}\right)$ captures both the cyclic homology $\overline{\mathrm{HC}}(A)$ and the Karoubi-de Rham homology $\overline{\mathrm{HD}}(A)$ at the same time.

5.1. Extended noncommutative de Rham complex. Let $B *_{\mathbf{k}} C$ denote the free product of unital algebras $B$ and $C$ over k. Define

$$
\Omega_{t}=\Omega_{t} A:=\Omega\left(A *_{\mathbf{k}} \mathbf{k}[t]\right) /(\mathbf{d} t) \cong \Omega A *_{\mathbf{k}} \mathbf{k}[t],
$$

where $(\mathrm{d} t)$ denotes the two-sided ideal generated by the element $\mathrm{d} t$. For all $p$ and $q$, let $\Omega_{t}^{p, q} \subseteq \Omega_{t}$ denote the subspace of de Rham degree $p$ and degree $q$ in $t$ (note that this notation differs from that of [GS]). The isomorphism on the right of (5.1.1) respects the bigradings.

Next, we define the commutator quotient

$$
\mathrm{DR}_{t}=\mathrm{DR}_{t} A:=\Omega_{t} /\left[\Omega_{t}, \Omega_{t}\right], \quad \mathrm{DR}_{t}=\bigoplus_{p, q} \mathrm{DR}_{t}^{p, q} \quad \text { where } \quad \mathrm{DR}_{t}^{p, q}=\left(\Omega_{t}^{p, q}\right)_{t} .
$$

As observed in [GS], for each $q \geq 1$, there is a canonical isomorphism $\operatorname{DR}_{t}^{\cdot, q} \cong \Omega^{\otimes q} /(\mathbb{Z} / q)$. This yields a direct sum decomposition

$$
\mathrm{DR}_{t}=\bigoplus_{q \geq 0} \mathrm{DR}_{t}^{\cdot, q} \cong \mathrm{DR} \oplus \Omega \oplus \Omega^{\otimes 2} /(\mathbb{Z} / 2) \oplus \cdots .
$$

In particular, there is a canonical isomorphism $\iota: \operatorname{DR}_{t}^{\bullet, 1}=\left(t \Omega^{\bullet}\right)_{\natural} \stackrel{\sim}{\rightarrow} \Omega^{\bullet}$.

One has a natural differential $\mathrm{d}: \Omega_{t}^{\cdot, q} \rightarrow \Omega_{t}^{\cdot+1, q}$ that restricts to the ordinary Karoubi-de Rham differential on $\Omega$, and kills $t$. One also has a derivation $\frac{d}{d t}: \Omega_{t}^{p, \bullet} \rightarrow \mathrm{DR}_{t}^{p, \bullet-1}$ that kills $\Omega \subset \Omega_{t}$ and sends $t$ to 1 . These maps descend to $\mathrm{DR}_{t}$.

There are also reduced versions of all the above. In particular, $\bar{\Omega}_{t}=\bar{\Omega}_{t} A:=\Omega_{t} / \mathbf{k}[t]$ and $\overline{\mathrm{DR}}_{t}=$ $\overline{\mathrm{DR}}_{t} A:=\bar{\Omega}_{t} / \overline{\left[\Omega_{t}, \Omega_{t}\right]}=\mathrm{DR}_{t} / \mathbf{k}[t]$. Further, we put $\overline{\mathrm{DR}}_{t}^{+}:=\oplus_{q>0} \overline{\mathrm{DR}}^{\cdot, q}=\left(t \bar{\Omega}_{t}\right)_{\natural} \subseteq \overline{\mathrm{DR}}_{t}$.

Lemma 5.1.3. The complex $\left(\overline{\mathrm{DR}}_{t}^{+}\right.$, d) is acyclic.

Proof. From (5.1.2), we see that $\mathrm{DR}_{t}^{+}=\Omega \oplus \Omega^{\otimes 2} /(\mathbb{Z} / 2) \oplus \cdots$. Now, $H(\Omega, \mathrm{d}) \cong \mathbf{k}$, spanned by the natural inclusion $\mathbf{k} \subseteq \Omega$. Taking coinvariants with respect to the cyclic group $\mathbb{Z} / q$ is exact for all $q \geq 1$, thanks to our assumption that $\mathbf{k} \supseteq \mathbb{Q}$. Hence, $H\left(\mathrm{DR}_{t}^{+}, \mathrm{d}\right)=\bigoplus_{q \geq 1} \mathbf{k}^{\otimes q}$, spanned by the inclusions $\mathbf{k}^{\otimes q} \cong \mathbf{k} \cdot\left(t^{q}\right)_{\natural} \subseteq \mathrm{DR}_{t}^{+}$. The result now follows from the long exact sequence on homology associated to the short exact sequence $0 \rightarrow t \cdot \mathbf{k}[t] \rightarrow \mathrm{DR}_{t}^{+} \rightarrow \overline{\mathrm{DR}}_{t}^{+} \rightarrow 0$.

As an immediate consequence of Lemma 5.1.3 we obtain

Corollary 5.1.4. The natural projection $\overline{\mathrm{DR}}_{t} \rightarrow \overline{\mathrm{DR}}_{t} / \overline{\mathrm{DR}}_{t}^{+}=\overline{\mathrm{DR}}$, modulo $t$, induces an isomorphism $H\left(\overline{\mathrm{DR}}_{t}, \mathrm{~d}\right) \stackrel{\sim}{\rightarrow} H(\overline{\mathrm{DR}}, \mathrm{d})$, of cohomology. 
5.2. Following [GS], we observe that the derivation $(-\operatorname{ad} t)$ of the algebra $A *_{\mathbf{k}} \mathbf{k}[t]$ naturally gives rise to a contraction operation $\mathbf{i}_{t}: \Omega_{t}^{p, q} \rightarrow \Omega_{t}^{p-1, q+1}$. By definition, the map $\mathbf{i}_{t}$ is the (super) derivation of the algebra $\Omega_{t}$ that acts on generators by

$$
\mathbf{i}_{t}: t \mapsto 0, \quad \Omega^{0}=A \mapsto 0, \quad \Omega^{1} \supset \mathrm{d} A \ni \mathrm{d} a \mapsto[a, t] \quad \forall a \in A .
$$

One checks that $\mathbf{i}_{t}^{2}=0$ and that $\mathbf{i}_{t}$ descends to a map $\mathrm{i}_{t}: D \mathrm{D}_{t} \rightarrow D \mathrm{R}_{t}$. The operations $\mathbf{i}_{t}$ and $\mathrm{i}_{t}$ also descend to the corresponding reduced versions.

We are now able to give, following [GS], a more conceptual definition of the map i : DR $\rightarrow \Omega^{\bullet-1}$ that was introduced in $\$ 2$ using an explicit formula (2.1.3). Specifically, one easily checks that

$$
\mathrm{i}=\iota \circ \mathrm{i}_{t}, \quad \text { as maps } \mathrm{DR}^{\bullet}=\mathrm{DR}_{t}^{\bullet, 0} \longrightarrow \Omega^{\bullet-1}=\mathrm{DR}_{t}^{\cdot-1,1} .
$$

It follows immediately that the differentials $d$ and $\mathbf{i}_{t}$ (hence, also $i_{t}$ ) commute with $\frac{d}{d t}$, and that the differentials $d$ and $i_{t}$, on $\overline{\mathrm{DR}}_{t}$, anti-commute (see [CBEG] and [GS] for the latter fact).

5.3. The Tsygan map. Let $\overline{\mathrm{DR}}_{\mathrm{t}}^{\wedge}=\overline{\mathrm{DR}}_{\mathrm{t}}^{\wedge}(A)^{\bullet}:=\prod_{q \geq 0} \overline{\mathrm{DR}}_{t}^{\cdot, q}$ be the $t$-adic completion of $\overline{\mathrm{DR}}_{t}$. We define the Tsygan map as a k-linear map $\mathrm{E}: \bar{\Omega} \rightarrow \overline{\mathrm{DR}}_{\mathrm{t}}^{\wedge} \cdot$ given, for any $a_{0}, a_{1}, \ldots, a_{n} \in A$, by

$$
\mathrm{E}\left(a_{0} \mathrm{~d} a_{1} \cdots \mathrm{d} a_{n}\right)=\sum_{k_{0}, \ldots, k_{n} \geq 0} \frac{1}{\left(k_{0}+\cdots+k_{n}+n\right) !}\left(a_{0} t^{k_{0}} \mathrm{~d} a_{1} t^{k_{1}} \cdots \mathrm{d} a_{n} t^{k_{n}}\right) \text { 七. }
$$

The map $\left.\mathrm{E}\right|_{\bar{\Omega}^{n}}$ may be viewed as a noncommutative analogue of multiplication by $\frac{1}{n !} \exp (t)$. To explain this, we view the algebra $\Omega \otimes \mathbf{k}[t]$ as a quotient of the algebra $\Omega *_{\mathbf{k}} \mathbf{k}[t]$ by the two-sided ideal generated by $[t, \Omega]$. Thus, one has the natural algebra homomorphism $\Omega_{t} \rightarrow \Omega[t]$ that 'makes the variable $t$ central.' This homomorphism clearly descends to a linear map $\overline{\mathrm{DR}}_{t} \rightarrow \overline{\mathrm{DR}}[t]$. The latter map can further be extended, by continuity, to a map $c_{t}: \overline{\mathrm{DR}}_{\mathrm{t}}^{\wedge} \rightarrow \overline{\mathrm{DR}} \llbracket t \rrbracket$, between the corresponding $t$-adic completions. Then, one has

Lemma 5.3.2. For every $f \in \bar{\Omega}^{n}$, in $\overline{\mathrm{DR}} \llbracket t \rrbracket$ we have: $\mathrm{c}_{t} \circ \mathrm{E}(f)=\frac{1}{n !} \cdot \exp (t) f_{\natural}$.

Proof. Given positive integers $k$ and $n$, let $P_{n}(k)$ denote the number of partitions $k_{0}+\cdots+k_{n}=k$, of $k$ into $n+1$ parts. That number is given by the formula $P_{n}(k)=\frac{(k+n) !}{k ! n !}$. Using this and the definition of the map $\mathrm{E}$, for every $f=a_{0} \mathrm{~d} a_{1} \cdots \mathrm{d} a_{n} \in \bar{\Omega}^{n}$, we compute

$$
\begin{gathered}
\mathrm{c}_{t} \circ \mathrm{E}(f)=\sum_{k_{0}, \ldots, k_{n} \geq 0} \frac{1}{\left(k_{0}+\cdots+k_{n}+n\right) !} \cdot \mathrm{c}_{t}\left(\left(a_{0} t^{k_{0}} \mathrm{~d} a_{1} t^{k_{1}} \cdots \mathrm{d} a_{n} t^{k_{n}}\right)_{\natural}\right) \\
\sum_{k_{0}, \ldots, k_{n} \geq 0} \frac{1}{\left(k_{0}+\cdots+k_{n}+n\right) !} \cdot t^{k_{0}+\ldots+k_{n}}\left(a_{0} \mathrm{~d} a_{1} \cdots \mathrm{d} a_{n}\right)_{\natural}=\sum_{k \geq 0} P_{n}(k) \frac{1}{(k+n) !} \cdot t^{k} f_{\natural} \\
\quad=\sum_{k \geq 0} \frac{(k+n) !}{k ! n !} \frac{1}{(k+n) !} \cdot t^{k} f_{\natural}=\sum_{k \geq 0} \frac{1}{k ! n !} \cdot t^{k} f_{\natural}=\frac{1}{n !} \cdot \exp (t) f_{\natural} .
\end{gathered}
$$

Theorem 5.3.3. The map $\mathrm{E}$ has the properties:

$$
\text { (i) } \frac{d}{d t} \circ \mathrm{E}=\mathrm{E} ; \quad \text { (ii) } \mathrm{E} \circ \mathrm{B}=\mathrm{d} \circ \mathrm{E} ; \quad \text { (iii) } \mathrm{E} \circ \mathrm{b}=\mathrm{i}_{t} \circ \mathrm{E} \text {. }
$$

This theorem will be proved in $\$ 5.4$.

Remark 5.3.5. We have arrived at the definition of the map E by analyzing a construction of Tsygan [Tsy2] (that construction was, in its turn, motivated by our results [GS]). In particular, equations (5.3.4)(ii)-(iii) are an adaptation of the main result of [Tsy2]. It is likely that a proof of these equations, which is more conceptual than the one given in \$5.4 below, can be extracted from [Tsy2], although the arguments in op. cit. are written in the setting of the standard (i.e., commutative) 
equivariant cochain complex rather than the extended noncommutative de Rham complex. However, such a noncommutative adaptation of the argument of [Tsy2] would be less direct since it would have to go through an auxiliary, much larger, complex $\bar{\Omega}(\Omega A)$ (this would allow one to write an analogue of $E$ as an exponential of a certain contraction map on this larger complex and to apply the noncommutative calculus developed in [TT]).

Remark 5.3.6. One can use (5.3.4)(i) to find the coefficients of the map $\mathrm{E}$ in the summation (5.3.1). Although one might expect that these coefficients should be $\frac{1}{\operatorname{deg}_{t} !}$, inspecting the LHS of (5.3.4)(i) more carefully one sees that it must be $\frac{1}{\left(\operatorname{leg}_{t}+\operatorname{deg}_{\mathrm{DR}}\right) !}$ as indicated.

Remark 5.3.7. Restricting equations (5.3.4)(ii)-(iii) to the harmonic part of $\bar{\Omega}$ and using the chain map $\mathrm{N}$ !, defined as $\mathrm{N} ! \bar{\Omega}^{n}=n ! \mathrm{Id}$, one obtains $\left.\mathrm{E}\right|_{P \bar{\Omega}} \mathrm{N} ! \circ \mathrm{d}=\left.\mathrm{d} \circ \mathrm{E}\right|_{P \bar{\Omega}} \mathrm{N} !$ and $\left.\mathrm{E}\right|_{P \bar{\Omega}} \mathrm{N} ! \circ \mathbf{i}=\left.\mathrm{i}_{t} \circ \mathrm{E}\right|_{P \bar{\Omega}} \mathrm{N}$.

Curiously, we do not know if there is any explicit formula, without using harmonic decomposition, for a map $\mathrm{E}^{\prime}: \bar{\Omega} \rightarrow \overline{\mathrm{DR}}_{\mathrm{t}}^{\wedge}$ which satisfies $\mathrm{E}^{\prime} \circ \mathrm{d}=\mathrm{d} \circ \mathrm{E}^{\prime}$ and $\mathrm{E}^{\prime} \circ \mathbf{i}=\mathrm{i}_{t} \circ \mathrm{E}^{\prime}$. (To define such a map using harmonic decomposition, one could set $\mathrm{E}^{\prime}=\mathrm{E} \mathrm{N} ! P$.)

Perhaps this is related to the fact that (the antiharmonic part of) the bicomplex $(\bar{\Omega}, \mathrm{d}, \mathbf{i})$ is somewhat badly behaved, i.e., the homologies of $(\bar{\Omega} \otimes R, \mathbf{i}-u \mathbf{d})$ and $(\bar{\Omega} \llbracket u \rrbracket, \mathbf{i}-u \mathrm{~d})$ pick up the huge extra factor $\overline{[\mathrm{d} \Omega, \mathrm{d} \Omega]}$ (see Theorem 3.2.1). On the other hand, we will see in $\$ 5.5$ and 97 below that the homologies of $\left(\overline{\mathrm{DR}}_{t} \otimes R, \mathrm{i}_{t}-u \mathrm{~d}\right)$ and $\left(\overline{\mathrm{DR}}_{t} \llbracket u \rrbracket, \mathrm{i}_{t}-u \mathrm{~d}\right)$ are nicely expressible in terms of ordinary cyclic and Karoubi-de Rham homology. This suggests that the bicomplex $\left(\overline{\mathrm{DR}}_{t}, \mathrm{i}_{t}, \mathrm{~d}\right)$ should indeed be more closely related to $(\bar{\Omega}, \mathrm{b}, \mathrm{B})$ than to $(\bar{\Omega}, \mathbf{i}, \mathrm{d})$.

Next, we recall that the differentials $B$ and $b$, as well as $d$ and $i_{t}$, anti-commute. Therefore, thanks to Theorem 5.3.3. the map E gives a morphism $(\bar{\Omega}, \mathrm{B}, \mathrm{b}) \rightarrow\left(\overline{\mathrm{DR}}_{\mathrm{t}}^{\wedge}, \mathrm{d}, \mathrm{i}_{t}\right)$, of bicomplexes. This induces maps $H^{\bullet}(\bar{\Omega}, \mathrm{b}) \rightarrow H^{\bullet}\left(\overline{\mathrm{DR}}_{\mathrm{t}}^{\wedge}, \mathrm{i}_{t}\right)$ and $H^{\bullet}(\bar{\Omega} / \mathrm{B} \bar{\Omega}, \mathrm{b}) \rightarrow H^{\bullet}\left(\overline{\mathrm{DR}}_{\mathrm{t}}^{\wedge} / \mathrm{d}_{\mathrm{DR}_{\mathrm{t}}}^{\wedge}, \mathrm{i}_{t}\right)$ on homology.

Observe that, since the differential $\mathrm{i}_{t}$ is homogeneous in $t$, each individual homology group of the complexes $\left(\overline{\mathrm{DR}}_{t}, \mathrm{i}_{t}\right)$ and $\left(\overline{\mathrm{DR}}_{t} / \mathrm{d} \overline{\mathrm{DR}}_{t}, \mathrm{i}_{t}\right)$ has an additional grading

$$
H^{p}\left(\overline{\mathrm{DR}}_{t}, \mathrm{i}_{t}\right)=\oplus_{q} H\left(\overline{\mathrm{DR}}_{t}, \mathrm{i}_{t}\right)^{p, q} \text {, and } H^{p}\left(\overline{\mathrm{DR}}_{t} / \mathrm{d} \overline{\mathrm{DR}}_{t}, \mathrm{i}_{t}\right)=\oplus_{q} H\left(\overline{\mathrm{DR}}_{t} / \mathrm{d} \overline{\mathrm{DR}}_{t}, \mathrm{i}_{t}\right)^{p, q} \text {, }
$$

where $p$ refers to the de Rham degree and $q$ refers to degree in $t$. Therefore, the map E produces, by separating degrees in $t$, an infinite collection $\left\{\mathrm{E}^{q}, q=0,1, \ldots\right\}$ of maps

$$
H^{\bullet}(\bar{\Omega}, \mathrm{b}) \stackrel{\mathrm{E}^{q}}{\longrightarrow} H^{\bullet}\left(\overline{\mathrm{DR}}_{t}, \mathrm{i}_{t}\right)^{\bullet, q} \text {, and } H^{\bullet}(\bar{\Omega} / \mathrm{B} \bar{\Omega}, \mathrm{b}) \stackrel{\mathrm{E}^{q}}{\longrightarrow} H\left(\overline{\mathrm{DR}}_{t} / \mathrm{d} \overline{\mathrm{DR}}_{t}, \mathrm{i}_{t}\right)^{\bullet, q} .
$$

For $q=0$, the above maps reduce to the maps $f \mapsto f_{\natural}$ and $f \bmod (\operatorname{im} \mathrm{B}) \mapsto f_{\natural} \bmod (\operatorname{imd})$.

Observe further that the map $\frac{d}{d t}$ commutes with $\mathrm{d}_{\text {and }} \mathrm{i}_{t}$, and hence it induces endomorphisms of the complex $\left(\overline{\mathrm{DR}}_{t}, \mathrm{i}_{t}\right)$ and $\left(\overline{\mathrm{DR}}_{t} / \mathrm{d} \overline{\mathrm{DR}}_{t}, \mathrm{i}_{t}\right)$. On homology, this gives maps

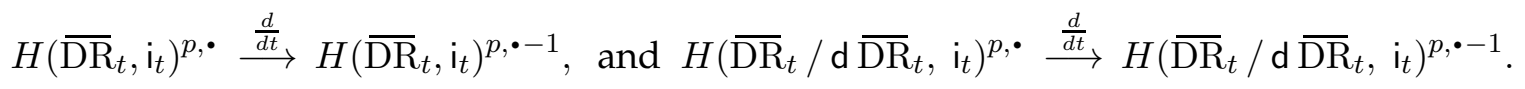

It is clear from (5.3.4) (i) that the maps $\mathrm{E}^{q}, q \geq 0$, satisfy a chain of relations

$$
\frac{d^{k}}{d t^{k}} \circ \mathrm{E}^{q}=\mathrm{E}^{q-k}, \quad \text { for every } k \leq q .
$$

Our second important result about the Tsygan map, that will be proved in section 6 reads

Theorem 5.3.10. Each of the maps in (5.3.8) is an isomorphism, for every $q \geq 0$.

5.4. Proof of Theorem 5.3.3. Equation (5.3.4)(i) follows from the expansion

$$
\frac{d}{d t}\left(t^{k_{0}} a_{0} t^{k_{1}} \mathrm{~d} a_{1} \ldots t^{k_{n}} \mathrm{~d} a_{n}\right)=\sum_{j} k_{j} t^{k_{0}} a_{0} \ldots t^{k_{j-1}} \mathrm{~d} a_{j-1} t^{k_{j}-1} \mathrm{~d} a_{j} t^{k_{j+1}} \cdots t^{k_{n}} \mathrm{~d} a_{n}
$$

generalizing the proof that $\frac{d}{d t} \exp (t)=\exp (t)$, in terms of the expansion $\exp (t)=\sum_{m \geq 0} \frac{1}{m !} t^{m}$.

To prove (5.3.4)(ii), we compute 


$$
\begin{aligned}
\mathrm{E} \circ \mathrm{B}\left(a_{0} \mathrm{~d} a_{1} \cdots \mathrm{d} a_{n}\right)= & \sum_{j=0}^{n} \sum_{k_{0}, \ldots, k_{n+1}} \frac{1}{\left(k_{0}+\cdots+k_{n+1}+(n+1)\right) !}\left(t^{k_{0}} \mathrm{~d} a_{j} t^{k_{1}} \mathrm{~d} a_{j+1} \cdots t^{k_{n}} \mathrm{~d} a_{j-1} t^{k_{n+1}}\right)_{\natural} \\
= & \sum_{k_{0}, \ldots, k_{n}} \frac{1}{\left(k_{0}+\cdots+k_{n}+n\right) !}\left(\mathrm{d} a_{0} t^{k_{0}} \cdots \mathrm{d} a_{n} t^{k_{n}}\right)_{\natural}=\mathrm{d} \circ \mathrm{E}\left(a_{0} \mathrm{~d} a_{1} \cdots \mathrm{d} a_{n}\right) .
\end{aligned}
$$

Here and below, the indices $k_{0}, \ldots, k_{n+1}$ always run over all nonnegative integers.

To prove (5.3.4)(iii) we will use the identities, for every $n \geq 1$ :

$$
\begin{gathered}
\sum_{p, q \geq 0} \frac{t^{p}[t, a] t^{q}}{(p+q+n) !}=\sum_{p, q \geq 0} \frac{t^{p+1} a t^{q}-t^{p} a t^{q+1}}{(p+q+n) !}=\quad(\text { taking } k:=p+1 \text { and } \ell:=q+1) \\
=\left(\sum_{k, q \geq 0} \frac{t^{k} a t^{q}}{(k-1+q+n) !}-\sum_{q \geq 0} \frac{a t^{q}}{(q+n-1) !}\right)-\left(\sum_{p, \ell \geq 0} \frac{t^{p} a t^{\ell}}{(p+\ell-1+n) !}-\sum_{p \geq 0} \frac{t^{p} a}{(p+n-1) !}\right) \\
=\sum_{p \geq 0} \frac{t^{p} a}{(p+n-1) !}-\sum_{q \geq 0} \frac{a t^{q}}{(q+n-1) !}=\sum_{j \geq 0} \frac{t^{j} a-a t^{j}}{(j+n-1) !} .
\end{gathered}
$$

Using the above formula, for every $a_{0}, \ldots, a_{n} \in A$, in $\bar{\Omega}_{t}$, we find

$$
\begin{aligned}
& \sum_{j=1}^{n} \sum_{k_{0}, \ldots, k_{n}} \frac{(-1)^{j}}{\left(k_{0}+\cdots+k_{n}+n\right) !} a_{0} t^{k_{0}} \mathrm{~d} a_{1} t^{k_{1}} \cdots \mathrm{d} a_{j-1} t^{k_{j-1}}\left[t, a_{j}\right] t^{k_{j}} \mathrm{~d} a_{j+1} t^{k_{j+1}} \cdots \mathrm{d} a_{n} t^{k_{n}} \\
= & \sum_{j=1}^{n} \sum_{k_{0}, \ldots, \hat{k}_{j}, \ldots, k_{n}} \frac{(-1)^{j}}{\left(k_{0}+\cdots \hat{k}_{j} \cdots+k_{n}+(n-1)\right) !}\left(a_{0} t^{k_{0}} \mathrm{~d} a_{1} t^{k_{1}} \cdots \mathrm{d} a_{j-1} t^{k_{j-1}} a_{j} \mathrm{~d} a_{j+1} t^{k_{j+1}} \cdots \mathrm{d} a_{n} t^{k_{n}}\right. \\
= & \left.-a_{0} t^{k_{0}} \mathrm{~d} a_{1} t^{k_{1}} \cdots \mathrm{d} a_{j-1} a_{j} t^{k_{j-1}} \mathrm{~d} a_{j+1} t^{k_{j+1}} \cdots \mathrm{d} a_{n} t^{k_{n}}\right) \\
& +\sum_{j=1}^{n-1}\left(\sum_{k_{0}, \ldots, \hat{k}_{j}, \ldots, k_{n}} \frac{(-1)^{j}}{\left(k_{0}+\cdots, \hat{k}_{j} \cdots+k_{n}\right.} a_{0} t^{k_{0}} \mathrm{~d} a_{1} \cdots \mathrm{d} a_{j-1} t^{k_{j-1}} \mathrm{~d}\left(a_{j} a_{j+1}\right) t^{k_{j+1}} \cdots \mathrm{d} a_{n} t^{k_{n}}\right) \\
& +\frac{1}{\left(k_{1}+\cdots+k_{n}+(n-1)\right) !}\left(a_{0} a_{1} t^{k_{1}} \mathrm{~d} a_{2} t^{k_{2}} \cdots \mathrm{d} a_{n} t^{k_{n}}+(-1)^{n} a_{0} t^{k_{1}} \mathrm{~d} a_{1} t^{k_{2}} \cdots \mathrm{d} a_{n-1} t^{k_{n}} a_{n}\right) .
\end{aligned}
$$

Now, by cyclic symmetry, in $\overline{\mathrm{DR}}_{t}$,

$$
\left(a_{0} t^{k_{1}} \mathrm{~d} a_{1} t^{k_{2}} \cdots \mathrm{d} a_{n-1} t^{k_{n}} a_{n}\right)_{\natural}=\left(a_{n} a_{0} t^{k_{1}} \mathrm{~d} a_{1} t^{k_{2}} \cdots \mathrm{d} a_{n-1} t^{k_{n}}\right)_{\mathfrak{\natural}} .
$$

Therefore, the image in $\overline{\mathrm{DR}}_{t}$ of the expression in the last two lines of (5.4.1) may be written as

$$
\begin{aligned}
\mathrm{E}\left(\sum_{j=1}^{n-1}(-1)^{j} a_{0} \mathrm{~d} a_{1} \cdots \mathrm{d} a_{j-1} \mathrm{~d}(\right. & \left.\left.a_{j} a_{j+1}\right) \mathrm{~d} a_{j+2} \cdots \mathrm{d} a_{n}\right) \\
+ & \mathrm{E}\left(a_{0} a_{1} \mathrm{~d} a_{2} \cdots \mathrm{d} a_{n}\right)+(-1)^{n} \mathrm{E}\left(a_{n} a_{0} \mathrm{~d} a_{1} \mathrm{~d} a_{2} \cdots \mathrm{d} a_{n-1}\right) .
\end{aligned}
$$


Further, the standard formula for the Hochschild differential written in terms of differential forms [CQ1, formula (10)] reads:

$$
\begin{aligned}
\mathrm{b}\left(a_{0} \mathrm{~d} a_{1} \cdots \mathrm{d} a_{n}\right)=\sum_{j=1}^{n-1}(-1)^{j} a_{0} \mathrm{~d} a_{1} \cdots \mathrm{d} a_{j-1} \mathrm{~d}\left(a_{j} a_{j+1}\right) \mathrm{d} a_{j+2} \cdots \mathrm{d} a_{n} \\
\quad+a_{0} a_{1} \mathrm{~d} a_{2} \cdots \mathrm{d} a_{n}+(-1)^{n} a_{n} a_{0} \mathrm{~d} a_{1} \mathrm{~d} a_{2} \cdots \mathrm{d} a_{n-1} .
\end{aligned}
$$

Thus, combining everything together and using that the expression in the top line of (5.4.1) equals $\mathrm{i}_{t} \circ \mathrm{E}\left(a_{0} \mathrm{~d} a_{1} \cdots \mathrm{d} a_{n}\right)$, we deduce that $\mathrm{i}_{t} \circ \mathrm{E}\left(a_{0} \mathrm{~d} a_{1} \cdots \mathrm{d} a_{n}\right)=\mathrm{E} \circ \mathrm{b}\left(a_{0} \mathrm{~d} a_{1} \cdots \mathrm{d} a_{n}\right)$.

5.5. Extended cyclic homology. It will be convenient to introduce an additional parameter $u$, and consider the $\mathbf{k}[u]$-module $\overline{\mathrm{DR}}_{t} \otimes R$. We equip this module with two different gradings. The first grading, referred to as homological grading, is defined by assigning the component $\overline{\mathrm{DR}}_{t}^{p, q} \cdot u^{-r}$ homological degree $\ell=p+2 r$. Thus, the $u$ variable has homological degree -2 . The $t$ parameter has homological degree zero, so it does not contribute to the homological grading. The second grading, referred to as internal grading, is defined by assigning the component $\overline{\mathrm{DR}}_{t}^{p, q} \cdot u^{-r}$ internal degree $p+q+r$.

On $\overline{\mathrm{DR}}_{t} \otimes R$, we have a pair of anti-commuting differentials, $u \mathrm{~d}$ and $\mathrm{i}_{t}$. Each of these differentials has homological degree -1 and internal degree zero. We define $\overline{\mathrm{EHC}}(A)$, the reduced extended cyclic homology of $A$, as

$$
\overline{\mathrm{EHC}}_{\ell}(A)=H^{\ell}\left(\overline{\mathrm{DR}}_{t} \otimes R, \mathrm{i}_{t}-u \mathrm{~d}\right),
$$

where $\ell$ on the RHS stands for homological degree.

Since the internal grading is preserved by both differentials, it provides a direct sum decomposition of complexes,

$$
\overline{\mathrm{DR}}_{t} \otimes R=\bigoplus_{k}\left(\overline{\mathrm{DR}}_{t} \otimes R\right)_{k}, \quad\left(\overline{\mathrm{DR}}_{t} \otimes R\right)_{k}:=\bigoplus_{p+q+r=k} \overline{\mathrm{DR}}^{p, q} \cdot u^{-r},
$$

where, $\left(\overline{\mathrm{DR}}_{t} \otimes R\right)_{k}$, the homogeneous component of internal degree $k$, is a subcomplex of $\overline{\mathrm{DR}}_{t} \otimes R$ with the inherited differential $i_{t}-u d$ and inherited homological grading. We write $w_{k}: \overline{\mathrm{DR}}_{t} \otimes R \rightarrow$ $\left(\overline{\mathrm{DR}}_{t} \otimes R\right)_{k}$ for the projection to that homogeneous component.

For every integer $m$, we let

$$
\overline{\mathrm{EHC}}_{\ell}(A, m):=H^{\ell}\left(\left(\overline{\mathrm{DR}}_{t} \otimes R, \mathrm{i}_{t}-u \mathrm{~d}\right)_{\ell-m}\right) .
$$

Thus, one obtains a 'weight decomposition': $\overline{\operatorname{EHC}}_{\ell}(A)=\oplus_{m} \overline{\mathrm{EHC}}_{\ell}(A, m)$, for $m \in \mathbb{Z}$.

Our main result about extended cyclic homology is the following theorem. Part (i) of the theorem shows that the knowledge of the extended cyclic homology is equivalent to the knowledge of both the usual cyclic homology and the Karoubi-de Rham homology.

Theorem 5.5.2. (i) One has a canonical $\mathbf{k}[u]$-module isomorphism

$$
\overline{\mathrm{EHC}}(A) \cong\left(\overline{\mathrm{HD}}(A) \otimes u^{-1} \mathbf{k}\left[u^{-1}\right]\right) \oplus \overline{\mathrm{HC}}(A)[t],
$$

where the variable ' $u$ ' annihilates the second summand, and on $\overline{\mathrm{HD}}(A) \otimes u^{-1}$ restricts to the natural inclusion $\overline{\mathrm{HD}}(A) \hookrightarrow \overline{\mathrm{HC}}(A)$ into the second summand in degree zero in $t$. Furthermore, the group $\overline{\mathrm{EHC}}_{\ell}(A, m)$ vanishes for all $m>\left[\frac{\ell}{2}\right]$, and

$$
\overline{\operatorname{EHC}}_{\ell}(A, m) \cong \begin{cases}\overline{\mathrm{HD}}_{\ell-2 m}(A) & 0<m \leq\left[\frac{\ell}{2}\right], \\ \overline{\mathrm{HC}}_{\ell}(A) & m \leq 0 .\end{cases}
$$


(ii) For every $q \geq 0$, there are canonical graded space isomorphisms

$$
\begin{aligned}
& H\left(\overline{\mathrm{DR}}_{t}, \mathrm{i}_{t}\right)^{\bullet, q} \cong \overline{\mathrm{HH}} \cdot(A), \\
& H\left(\overline{\mathrm{DR}}_{t} / \mathrm{d} \overline{\mathrm{DR}}_{t}, \mathrm{i}_{t}\right)^{\bullet, q} \cong \overline{\mathrm{HC}} \cdot(A) .
\end{aligned}
$$

Remark 5.5.6. In the special case $q=0$, using the identification in (5.2.1), the above becomes

$$
\begin{aligned}
& H\left(\overline{\mathrm{DR}}_{t}, \mathrm{i}_{t}\right)^{\bullet, 0}=\operatorname{ker}\left(\mathrm{i}: \overline{\mathrm{DR}}^{\bullet} \rightarrow \bar{\Omega}^{-1}\right) \\
& H\left(\overline{\mathrm{DR}}_{t} / \mathrm{d} \overline{\mathrm{DR}}_{t}, \mathrm{i}_{t}\right)^{\bullet, 0}=\operatorname{ker}\left(\mathrm{i}: \overline{\mathrm{DR}}^{\bullet} / \mathrm{d} \overline{\mathrm{DR}}^{\cdot-1} \rightarrow \bar{\Omega}^{\bullet-1} / \mathrm{d} \bar{\Omega}^{\cdot-2}\right) .
\end{aligned}
$$

Therefore, for $q=0$, isomorphisms (5.5.4)-(5.5.5) may be equivalently rewritten as

$$
\begin{gathered}
\operatorname{ker}\left(\mathrm{i}: \overline{\mathrm{DR}}^{\bullet} \rightarrow \bar{\Omega}^{-1}\right) \cong \overline{\mathrm{HH}} \cdot(A), \\
\operatorname{ker}\left(\mathrm{i}: \overline{\mathrm{DR}}^{\cdot} / \mathrm{d}^{\cdot-1} \rightarrow \overline{\mathrm{DR}}^{\cdot-1} / \mathrm{d}^{\cdot} \bar{\Omega}^{-2}\right) \cong \overline{\mathrm{HC}} \cdot(A) .
\end{gathered}
$$

These are nothing but our isomorphisms (3.1.4)-(3.1.5). Thus, we see that Theorem 5.5.2(ii) incorporates Theorem 3.1.3, as the special case $q=0$.

5.6. Theorem 5.3.10 implies Theorem 5.5.2. We consider part (ii) of Theorem 5.5.2 first. The proof of this part involves the Tsygan map $E$ in a crucial way.

In more detail, the Hochschild homology of $A$ is computed by the complex $(\bar{\Omega}, \mathrm{b})$. Thus, the map $H(\bar{\Omega}, \mathrm{b}) \rightarrow H^{\bullet}\left(\overline{\mathrm{DR}}_{t}, \mathrm{i}_{t}\right)^{\bullet}, q$ in (5.3.8), which is an isomorphism thanks to Theorem 5.3.10, provides isomorphism (5.5.4). Similarly, in the cyclic homology case, we use Lemma 3.1.7. Thus, we define isomorphism (5.5.5) to be the composite of the isomorphism $\overline{\mathrm{HC}} \cdot(A) \stackrel{\sim}{\rightarrow} H^{\bullet}(\bar{\Omega} / \mathrm{B} \bar{\Omega}, \mathrm{b})$, of Lemma 3.1.7 with the map $\mathrm{E}^{q}$, where the latter is an isomorphism by Theorem 5.3.10.

For part (i), we first prove

Proposition 5.6.1. (i) The assignments

$$
\pi_{m}: \sum_{k \geq 0} f_{k} \cdot u^{-k} \mapsto f_{m} \bmod (t), \quad \pi_{-}: \sum_{k \geq 0} f_{k} \cdot u^{-k} \mapsto f_{0} \bmod (\mathrm{imd}),
$$

induce isomorphisms

$$
\begin{array}{lll}
\pi_{m}: \overline{\mathrm{EHC}}_{\ell}(A, m) \stackrel{\sim}{\rightarrow} \overline{\mathrm{HD}}_{\ell-2 m}(A), & m>0 ; \\
\pi_{-}: \overline{\operatorname{EHC}}_{\ell}(A, m) \stackrel{\sim}{\rightarrow} H\left(\overline{\mathrm{DR}}_{t} / \mathrm{d}_{\overline{\mathrm{DR}}}, \mathrm{i}_{t}\right)^{\ell,-m}, & m \leq 0 .
\end{array}
$$

(ii) For every $m \leq 0$, the following diagram commutes:

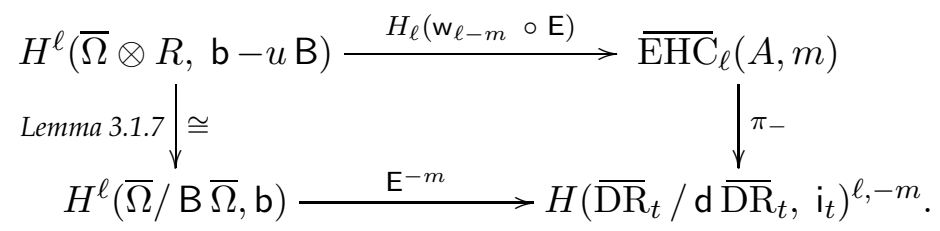

Proof. We view $\overline{\mathrm{DR}}_{t} \otimes R$ as a bicomplex $M$ of the form $M_{p, q}=\overline{\mathrm{DR}}_{t}^{p-q} \cdot u^{-q}$. The differentials are $\mathrm{i}_{t}: M_{p, q} \rightarrow M_{p-1, q}$ and $u \mathrm{~d}: M_{p, q} \rightarrow M_{p, q-1}$. We apply the spectral sequence associated with that bicomplex, by taking cohomology with respect to $u \mathrm{~d}$ first.

To compute the first page of the spectral sequence, we use the natural direct sum decomposition $\overline{\mathrm{DR}}_{t} \otimes R=\overline{\mathrm{DR}}_{t} \oplus u^{-1} \overline{\mathrm{DR}}_{t}\left[u^{-1}\right]$. For the cohomology of the first differential, this gives a direct sum decomposition $H\left(\overline{\mathrm{DR}}_{t} \otimes R, u \mathrm{~d}\right)=\overline{\mathrm{DR}}_{t} / \mathrm{d} \overline{\mathrm{DR}}_{t} \oplus u^{-1} H\left(\overline{\mathrm{DR}}_{t}, \mathrm{~d}\right)\left[u^{-1}\right]$. By Corollary 5.1.4, the projection modulo $t$ induces an isomorphism $H\left(\overline{\mathrm{DR}}_{t}, \mathrm{~d}\right) \stackrel{\sim}{\rightarrow} H(\overline{\mathrm{DR}}, \mathrm{d})$. Therefore, the first page of the spectral sequence takes the form $E_{1}=\overline{\mathrm{DR}}_{t} / \mathrm{d} \overline{\mathrm{DR}}_{t} \oplus u^{-1} H(\overline{\mathrm{DR}}, \mathrm{d})\left[u^{-1}\right]$. The differential $\mathrm{i}_{t}: E_{1} \rightarrow E_{1}$, on the first page, clearly increases degree in $t$ by 1 . Hence, this differential annihilates the direct summand $u^{-1} H(\overline{\mathrm{DR}}, \mathrm{d})\left[u^{-1}\right]$. We conclude that the second page of the spectral sequence 
reads $H\left(\overline{\mathrm{DR}}_{t} / \mathrm{d} \overline{\mathrm{DR}}_{t}, \mathrm{i}_{t}\right) \oplus u^{-1} H(\overline{\mathrm{DR}}, \mathrm{d})\left[u^{-1}\right]$, which is the right-hand side of the isomorphism of the proposition. It is immediate to see that the spectral sequence collapses at the second page. This proves part (i). Part (ii) follows directly from the formula for the map $\pi_{-}$.

To complete the proof of Theorem5.5.2(i), we note that the group that appears in the upper left corner of diagram (5.6.4) equals $\overline{\mathrm{HC}}_{\ell}(A)$, by (3.1.1). Thus, we may (and will) define isomorphism (5.5.3) by combining isomorphisms (5.5.5) and those of Proposition 5.6.1 together.

We conclude that the proof of Theorem 5.5.2 would be complete once we prove Theorem 5.3.10.

Remark 5.6.5. An analogue involving the maps $\pi_{m}$ of the statement that diagram (5.6.4) commutes will be given in Proposition 7.2.1(i) in \$7.

5.7. It is possible to write a relatively explicit formula for the inverse of the isomorphisms (5.6.2)(5.6.3). To this end, recall first that the complex $\left(\overline{\mathrm{DR}}_{t}^{+}, \mathrm{d}\right)$ is acyclic, by Lemma 5.1.3. Therefore, the Karoubi-de Rham differential d induces an isomorphism $\overline{\mathrm{DR}}_{t}^{+} / \mathrm{d} \overline{\mathrm{DR}}_{t}^{+} \stackrel{\sim}{\rightarrow} \mathrm{d} \overline{\mathrm{DR}}_{t}^{+}$.

Let $\tilde{\mathrm{d}}^{-1}: \mathrm{d} \overline{\mathrm{DR}}_{t}^{+} \rightarrow \overline{\mathrm{DR}}_{t}^{+}$be a (set-theoretic) section of the surjection $\mathrm{d}: \overline{\mathrm{DR}}_{t}^{+} \rightarrow \mathrm{d} \overline{\mathrm{DR}}_{t}^{+}$(we use the same notation as for the similar map in $\$ 3.2$. The choice of $\tilde{d}^{-1}$ will not affect anything below, and we need it only to write formulas which are independent of this choice.

Proposition 5.7.1. The inverses of the isomorphisms (5.6.2) and (5.6.3) of Proposition 5.6.1 are induced respectively by the maps

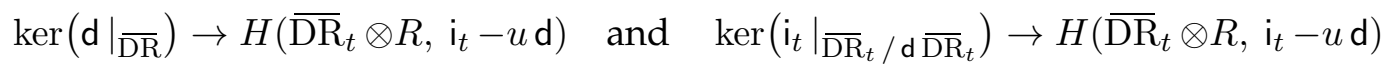

given by the assignments

$$
f \longmapsto u^{m}\left(\operatorname{Id}-u^{-1} \tilde{\mathrm{d}}^{-1} \mathrm{i}_{t}\right)^{-1}(f) \text { and } f \longmapsto\left(\operatorname{Id}-u^{-1} \tilde{\mathrm{d}}^{-1} \mathrm{i}_{t}\right)^{-1}(f) .
$$

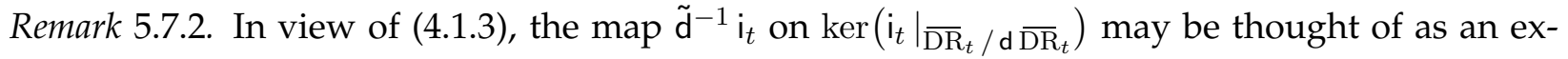
tended analogue of the periodicity operator $S$. Via Theorem 5.3.10, it actually becomes $S$ after making the above identification.

Proof. The statement of the proposition is essentially a simple consequence of the construction of differentials in the spectral sequence of a double complex. Below, we explain the case of the map $\pi_{-}$. The case of the maps $\pi_{m}, m>0$, is similar (and even easier).

Let $\operatorname{ker}\left(\mathrm{d} \mathrm{i}_{t}\right)$ be the kernel of the map $\mathrm{d} \circ \mathrm{i}_{t}: \overline{\mathrm{DR}}_{t} \rightarrow \overline{\mathrm{DR}}_{t}$. We claim first that $\tilde{\mathrm{d}}^{-1} \mathrm{i}_{t}$ is a welldefined set-theoretical endomorphism of $\operatorname{ker}\left(d i_{t}\right)$. To see this, observe that $i_{t}\left(\overline{\mathrm{DR}}_{t}\right) \subset \overline{\mathrm{DR}}_{t}^{+}$. Hence, thanks to acyclicity of the complex $\left(\overline{\mathrm{DR}}_{t}^{+}, \mathrm{d}\right)$, see Lemma 5.1.3, we deduce that an element $x \in \overline{\mathrm{DR}}_{t}$ belongs to $\operatorname{ker}\left(\mathrm{di}_{t}\right)$ if and only if $\mathrm{i}_{t} x=\mathrm{d} y$, for some $y \in \overline{\mathrm{DR}}_{t}^{+}$. Thus, for such an $x$, the element $\tilde{\mathrm{d}}^{-1} \mathrm{i}_{t} x=\tilde{\mathrm{d}}^{-1} \mathrm{~d} y$ is indeed well-defined. Furthermore, we compute $\mathrm{di}_{t}\left(\tilde{\mathrm{d}}^{-1} \mathrm{i}_{t} x\right)=-\mathrm{i}_{t} \mathrm{~d}\left(\tilde{\mathrm{d}}^{-1} \mathrm{i}_{t} x\right)=$ $-\left(\mathrm{i}_{t}\right)^{2}(x)=0$. Thus, we have proved that $\tilde{\mathrm{d}}^{-1} \mathrm{i}_{t} x \in \operatorname{ker}\left(\mathrm{d} \mathrm{i}_{t}\right)$.

The operator $\tilde{\mathrm{d}}^{-1} \mathrm{i}_{t}$ on $\operatorname{ker}\left(\mathrm{d} \mathrm{i}_{t}\right)$ has de Rham degree -2 , and hence Id $-u^{-1} \tilde{\mathrm{d}}^{-1} \mathrm{i}_{t}$ is invertible. Explicitly, an inverse is given, for every $x \in \operatorname{ker}\left(\mathrm{di}_{t}\right)$, by the formula $\left(\operatorname{Id}-u^{-1} \tilde{\mathrm{d}}^{-1} \mathrm{i}_{t}\right)^{-1}(x)=$ $\sum_{k \geq 0} u^{-k}\left(\tilde{\mathrm{d}}^{-1} \mathrm{i}_{t}\right)^{k}(x)$, where the terms $\left(\tilde{\mathrm{d}}^{-1} \mathrm{i}_{t}\right)^{k}(x)$ vanish for all $k$ greater than half the de Rham degree of $x$. Further, using that $\mathrm{d} \tilde{\mathrm{d}}^{-1}=\mathrm{Id}$, we compute

$$
\begin{aligned}
\left(\mathrm{i}_{t}-u \mathrm{~d}\right)\left(\mathrm{Id}-u^{-1} \tilde{\mathrm{d}}^{-1} \mathrm{i}_{t}\right)^{-1}=\left(\mathrm{d} \tilde{\mathrm{d}}^{-1} \mathrm{i}_{t}-u \mathrm{~d}\right)\left(\mathrm{Id}-u^{-1} \tilde{\mathrm{d}}^{-1} \mathrm{i}_{t}\right)^{-1} \\
\quad=u \mathrm{~d}\left(u^{-1} \tilde{\mathrm{d}}^{-1} \mathrm{i}_{t}-\mathrm{Id}\right)\left(\mathrm{Id}-u^{-1} \tilde{\mathrm{d}}^{-1} \mathrm{i}_{t}\right)^{-1}=-u \mathrm{~d} .
\end{aligned}
$$

We see that for every $z$ in the image of the map $\left(\operatorname{Id}-u^{-1} \tilde{\mathrm{d}}^{-1} \mathrm{i}_{t}\right)^{-1}$, the element $\left(\mathrm{i}_{t}-u \mathrm{~d}\right)(z)$ belongs to $u \overline{\mathrm{DR}}_{t}$. It follows that $z \bmod u \overline{\mathrm{DR}}_{t} \llbracket u \rrbracket$ is a cycle in the complex $\left(\overline{\mathrm{DR}}_{t} \otimes R, \mathrm{i}_{t}-u \mathrm{~d}\right)$. Thus, 
we have constructed the map

$$
\left(\mathrm{Id}-u^{-1} \tilde{\mathrm{d}}^{-1} \mathrm{i}_{t}\right)^{-1}: \operatorname{ker}\left(\mathrm{d} \mathrm{i}_{t}\right) \longrightarrow H\left(\overline{\mathrm{DR}}_{t} \otimes R, \mathrm{i}_{t}-u \mathrm{~d}\right)
$$

Now, it is immediate from the above construction that the above constructed map is a right inverse to the map $\pi_{-}$in the sense that, for every $x \in \operatorname{ker}\left(\mathrm{di}_{t}\right)$, the class of the element $x$ in $H\left(\overline{\mathrm{DR}}_{t} / \mathrm{d} \overline{\mathrm{DR}}_{t}, \mathrm{i}_{t}\right)$ equals $\pi_{-}\left(\mathrm{Id}-u^{-1} \tilde{\mathrm{d}}^{-1} \mathrm{i}_{t}\right)^{-1}(x)$. By definition, one has

$$
H\left(\overline{\mathrm{DR}}_{t} / \mathrm{d} \overline{\mathrm{DR}}_{t}, \mathrm{i}_{t}\right)=\frac{\operatorname{ker}\left(\mathrm{d} \mathrm{i}_{t}\right)}{\operatorname{kerd}+\mathrm{im} \mathrm{i}_{t}} .
$$

Therefore, for every $x \in$ ker $\mathrm{d}+\mathrm{im} \mathrm{i}_{t}$, we must have $\pi_{-}\left(\operatorname{Id}-u^{-1} \tilde{\mathrm{d}}^{-1} \mathrm{i}_{t}\right)^{-1}(x)=0$. Since $\pi_{-}$is an isomorphism, by Proposition 5.6.1, we deduce that the map $\left(\operatorname{Id}-u^{-1} \tilde{\mathrm{d}}^{-1} \mathrm{i}_{t}\right)^{-1}$ takes ker $\mathrm{d}+\mathrm{im} \mathrm{i}_{t}$ to zero on homology. We conclude that the map given in Proposition 5.7.1]is well defined. Moreover, it is a right inverse, and hence a two-sided inverse, to the isomorphism $\pi_{-}$.

\section{Proof of Theorem 5.3.10}

6.1. First of all, thanks to equation (5.3.9), $\frac{d^{q}}{d t^{q}} \circ \mathrm{E}^{q}=\mathrm{E}^{0}$. The maps $\mathrm{E}^{0}$ in (5.3.8) are isomorphisms by Theorem 3.1.3 together with Lemma 3.1.7. It follows that, for every $q \geq 0$, the map $\mathrm{E}^{q}$ is injective. Therefore, we only have to show that $\mathrm{E}^{q}$ is surjective.

To this end, we introduce a filtration on $\overline{\mathrm{DR}}_{t}$ by the degree in $A$ : precisely, the degree $\leq m$ part of the filtration is spanned by monomials in $t, A$, and $\mathrm{d} A$ with the elements of the latter two appearing at most $m$ times. This descends to filtrations under the surjections $\Omega_{t} \rightarrow \bar{\Omega}_{t} \rightarrow \overline{\mathrm{DR}}_{t}$. The differentials $i_{t}$ and $d$ preserve the resulting filtration. So we obtain a filtered bicomplex $\left(\overline{\mathrm{DR}}_{t}, \mathrm{~d}, \mathrm{i}_{t}\right)$.

It is immediate to check that $\operatorname{gr}\left(\overline{\mathrm{DR}}_{t}, \mathrm{~d}, \mathrm{i}_{t}\right)$, the associated graded bicomplex, is naturally isomorphic to $\left(\overline{\mathrm{DR}}_{t} A^{\prime}, \mathrm{d}, \mathrm{i}_{t}\right)$, where $A^{\prime}:=\bar{A} \oplus \mathbf{k}$ is the algebra with the trivial multiplication $\bar{A} \cdot \bar{A}=0$. Let $\mathrm{E}^{\prime}$ denote the Tsygan map for the algebra $A^{\prime}$. Clearly, one has that $\mathrm{E}^{\prime}=\mathrm{gr} \mathrm{E}$.

Thus, we have reduced the proof of the theorem to

Proposition 6.1.1. The maps $\left(\mathrm{E}^{\prime}\right)^{q}: H\left(\bar{\Omega} A^{\prime}, \mathrm{b}\right) \rightarrow H\left(\overline{\mathrm{DR}}_{t} A^{\prime}, \mathrm{i}_{t}\right)^{\bullet, q}$ and $\left(\mathrm{E}^{\prime}\right)^{q}: H\left(\bar{\Omega} A^{\prime} / \mathrm{B} \bar{\Omega} A^{\prime}, \mathrm{b}\right) \rightarrow$ $H\left(\overline{\mathrm{DR}}_{t} A^{\prime} / \mathrm{d} \overline{\mathrm{DR}}_{t} A^{\prime}, \mathrm{i}_{t}\right)^{\bullet, q}$ are both surjective for every $q \geq 0$.

The rest of $\sqrt{6}$ is devoted to the proof of this proposition. The idea of the proof is to reduce the result further to the special case where $\bar{A}=\mathbf{k}$ (and to lift from cyclic tensors to ordinary tensors). In this case, we compute the cohomology of the complexes $\left(\overline{\mathrm{DR}}_{t} A^{\prime}, \mathrm{i}_{t}\right)$ and $\left(\overline{\mathrm{DR}}_{t} A^{\prime} / \mathrm{d} \overline{\mathrm{DR}}_{t} A^{\prime}, \mathrm{i}_{t}\right)$ explicitly. More precisely, we will define, for each $\ell \geq 1$, a complex $C_{\ell}$ equipped with an action of $\mathbb{Z} / \ell$, the cyclic group. We identify the complex $\left(\overline{\mathrm{DR}}_{t} A^{\prime}, \mathrm{i}_{t}\right)$ with $\bigoplus_{\ell>1}\left(C_{\ell}\right)_{\mathbb{Z} / \ell}$, a direct sum of complexes of coinvariants of cyclic groups. We then compute explicitly the homology of each complex $C_{\ell}$. The computation is, however, quite technical; it will not be used elsewhere in the paper.

6.2. Linear algebra lemmas. We begin with a few elementary lemmas from linear algebra.

Let $V$ be a vector space over $\mathbb{R}$ equipped with a positive definite inner product $\langle-,-\rangle: V \times V \rightarrow$ $\mathbb{R}$. Recall that a linear operator $T: V \rightarrow V$ is called positive if it is self-adjoint and $\langle T v, v\rangle \geq 0$ for all $v \in V$. For a positive operator $T$ and $v \in V$, the equation $\langle T v, v\rangle=0$ implies that $T v=0$.

Lemma 6.2.1. Suppose that $T_{0}, \ldots, T_{k}$ are positive operators on vector spaces $V_{0}, \ldots, V_{k}$. Let $T:=$ $\sum_{i=0}^{k} \mathrm{Id}^{\otimes i} \otimes T_{i} \otimes \mathrm{Id}^{\otimes k-i}$. Then $T$ is positive, and $\operatorname{ker}(T)=\bigotimes_{i=0}^{k} \operatorname{ker}\left(T_{i}\right)$.

Proof. Clearly each $\mathrm{Id}^{\otimes i} \otimes T_{i} \otimes \mathrm{Id}^{\otimes k-i}$ is positive, and so the sum is positive. Since the kernel of $T$ is the space of vectors $v$ such that $\langle T v, v\rangle=0$, it is the intersection of the kernels of $\operatorname{Id}^{\otimes i} \otimes T_{i} \otimes \operatorname{Id}^{\otimes k-i}$, i.e., $\bigotimes_{i=0}^{k} \operatorname{ker}\left(T_{i}\right)$. 
Lemma 6.2.2. Let $B=\left(b_{i j}\right) \in \operatorname{Mat}_{n}(\mathbb{R})$ be a symmetric $n \times n$-matrix such that $b_{i i} \geq \sum_{j \neq i}\left|b_{i j}\right|$ for all $i$. Then $B$ is positive. Assume, in addition, that the graph obtained from $B$ with vertex set $\{1, \ldots, n\}$ and edges between $i$ and $j$ if $b_{i j} \neq 0$ is connected. Then $\operatorname{dim} \operatorname{ker}(B) \leq 1$. This kernel is nonzero if and only if:

- The equality $b_{i i}=\sum_{j \neq i}\left|b_{i j}\right|$ holds for all $i$;

- There exist signs $\lambda_{i} \in\{1,-1\}$ such that $\operatorname{sign}\left(b_{i j}\right)=-\lambda_{i} \lambda_{j}$ for all $i \neq j$.

Then the kernel is spanned by the vector $\left(\lambda_{1}, \ldots, \lambda_{n}\right)^{\top}$.

Here and below, we use the notation $\left(\lambda_{1}, \ldots, \lambda_{n}\right)^{\top}=\left(\begin{array}{c}\lambda_{1} \\ \vdots \\ \lambda_{n}\end{array}\right)$, where $\top$ stands for "transpose."

Proof. Let $v=\left(v_{1}, \ldots, v_{n}\right)^{\top}$. Then

$$
\langle B v, v\rangle=\sum_{i<j} b_{i j}\left(v_{i}^{2}+2 v_{i} v_{j}+v_{j}^{2}\right)+\sum_{i}\left(b_{i i}-\sum_{j \neq i} b_{i j}\right) v_{i}^{2} \geq 0 .
$$

Hence $B$ is positive. Now, suppose $B v=0$ and $v \neq 0$. Let $i$ be such that $\left|v_{i}\right| \geq\left|v_{j}\right|$ for all $j$. Then $0=b_{i i} v_{i}+\sum_{j \neq i} b_{i j} v_{j}$ and $b_{i i} \geq \sum_{j \neq i}\left|b_{i j}\right|$ implies that the equality holds, and that $\left|v_{j}\right|=\left|v_{i}\right|$ whenever $b_{i j} \neq 0$. Now assume that the graph of $B$ is connected. Then we conclude that $\left|v_{i}\right|$ are all equal. Hence the equality $b_{i i}=\sum_{j \neq i}\left|b_{i j}\right|$ holds for all $i$. Now, $B v=0$ if and only if $\operatorname{sign}\left(b_{i j}\right)=-v_{i} / v_{j}$ whenever $i \neq j$.

Remark 6.2.3. Lemma 6.2.2 can be significantly generalized: for arbitrary complex $B$ which is not necessarily symmetric, the condition $b_{i i} \geq \sum_{j \neq i}\left|b_{i j}\right|$ guarantees that the real parts of all eigenvalues of $B$ are nonnegative, and zero only for the zero eigenvalue. Let $Q(B)$ be a directed graph with vertex set $\{1,2, \ldots, n\}$ such that there is an edge from vertex $i$ to $j$ whenever $b_{i j} \neq 0$ and $i \neq j$. If the graph $Q(B)$ is strongly connected (i.e., there exists a directed path from every vertex to every other vertex), then $\operatorname{dim} \operatorname{ker}(B) \leq 1$ and it is nonzero if and only if $b_{i i}=\sum_{j \neq i}\left|b_{i j}\right|$ for all $i$ and there exist complex numbers $\lambda_{1}, \ldots, \lambda_{n}$ of absolute value 1 such that $b_{i j} /\left|b_{i j}\right|=-\lambda_{i} / \lambda_{j}$ whenever $b_{i j} \neq 0$. In this case the kernel is spanned by $\left(\lambda_{1}, \ldots, \lambda_{n}\right)^{\top}$.

Lemma 6.2.4. Suppose that $\left(V^{\bullet}, d\right)$ is a complex of vector spaces with $d: V^{\bullet} \rightarrow V^{\bullet+1}$. Suppose we are given an operator $h: V^{\bullet} \rightarrow V^{\bullet-1}$ such that

$$
V=\operatorname{ker}[h, d] \bigoplus \operatorname{im}[h, d], \quad \operatorname{ker} d=\operatorname{ker}\left(\left.[h, d]\right|_{\operatorname{ker} d}\right) \bigoplus \operatorname{im}\left(\left.[h, d]\right|_{\operatorname{ker} d}\right) .
$$

(E.g., this holds if $[h, d]$ acts semisimply on $V$ or its base change to an algebraically closed field.) Then, the inclusion $\left(\operatorname{ker}[h, d],\left.d\right|_{\operatorname{ker}[h, d]}\right) \rightarrow(V, d)$ is a quasi-isomorphism.

Proof. Since $(V, d)=\left(\operatorname{ker}[h, d],\left.d\right|_{\operatorname{ker}[h, d]}\right) \bigoplus\left(\operatorname{im}[h, d],\left.d\right|_{\operatorname{im}[h, d]}\right)$ is a direct sum of complexes, we only need to show that the second factor is acyclic. Since $\operatorname{ker}(d)=\left.\left.\operatorname{ker}(d)\right|_{\operatorname{ker}[h, d]} \oplus \operatorname{ker}(d)\right|_{\operatorname{im}[h, d]}$, and the first factor equals $\operatorname{ker}\left([h, d]||_{\operatorname{ker} d}\right)$, the assumption implies that $\left.\operatorname{ker}(d)\right|_{\operatorname{im}[h, d]}=\operatorname{im}\left(\left.[h, d]\right|_{\operatorname{ker} d}\right)=$ $\operatorname{im}\left(\left.d h\right|_{\operatorname{ker} d}\right)$. So every cycle in $\left(\operatorname{im}[h, d],\left.d\right|_{\operatorname{im}[h, d]}\right)$ is a boundary.

Remark 6.2.5. More generally, in Lemma 6.2.4 we could replace $\operatorname{ker}[h, d]$ and $\operatorname{im}[h, d]$ by $\operatorname{ker}[h, d]^{N}$ and $\operatorname{im}[h, d]^{N}$ for every $N \geq 1$. In particular, if $N \geq 1$ is such that $V=\bigoplus_{i \in \mathbb{Z}} V_{i}$ is a graded vector space with $\operatorname{dim} V_{i} \leq N$ for all $i$, then the conditions of the lemma will necessarily be satisfied with this modification.

In particular, Lemma 6.2.4 applies when $V^{\bullet}$ is a finite-dimensional complex of rational vector spaces and $[h, d]$ is a positive operator. 
6.3. An auxiliary complex. Our ultimate goal is to define a complex $C_{\ell}$ and compute its homology, as well as that of a certain quotient $C_{\ell} / d_{s} C_{\ell}$; here we begin, as an intermediate step, with a somewhat simpler complex. Consider the supercommutative algebra $S:=\mathbf{k}[s] /\left(s^{2}\right)$, with $s$ odd, and the left $S$-module

$$
M:=\operatorname{Span}\left(1, s, t^{i}, s t^{i}, t^{i} s, s t^{i} s \mid i \geq 1\right) \subset \mathbf{k}\langle s, t\rangle /\left(s^{2}\right) .
$$

Assign $M$ the grading in which 1 is odd, $s$ is even, and for all $i \geq 1, t^{i}$ and $s t^{i} s$ are even and $s t^{i}$ and $t^{i} s$ are odd. (In other words, we modify the natural grading from the algebra $\mathbf{k}\langle s, t\rangle /\left(s^{2}\right) \supset M$ by shifting the degree of the submodule $S \subset M$.) Moreover, equip $M$ with the right $S$-module structure $\cdot R$,

$$
(f) \cdot_{R} 1=f, \quad(f) \cdot_{R} s=-f s,
$$

which differs from the natural multiplication from the algebra $\mathbf{k}\langle s, t\rangle /\left(s^{2}\right)$ by a sign.

From now until the end of $\$ 6.7$, we will use simplified notation $\otimes=\otimes_{S}$. For convenience, equip $T_{S} M$ with a partially defined multiplication,

$$
\left(f_{1} \otimes \cdots \otimes f_{i}\right) \cdot\left(g_{1} \otimes \cdots \otimes g_{j}\right)=f_{1} \otimes \cdots \otimes f_{i} g_{1} \otimes \cdots \otimes g_{j},
$$

where here $f_{i} g_{1}$ is the multiplication in the ring $\mathbf{k}\langle s, t\rangle /\left(s^{2}\right)$ (not using $\cdot_{R}$ ). This is defined whenever $f_{i} g_{1} \in M$. Further, put $1_{m}:=1^{\otimes m}=1 \otimes 1 \otimes \cdots \otimes 1$ ( $m$ factors).

For each $\ell \geq 1$, we define a triple, $\tilde{d}_{s}, \tilde{\partial}^{0}, \tilde{\partial}^{1}$, of differentials on $M^{\otimes \ell}$, as follows. First, we set

$$
\begin{aligned}
\tilde{d}_{s} & : 1_{k} \mapsto s \cdot 1_{k} \mapsto 0, \\
\tilde{\partial}^{0}\left(1_{k}\right) & :=\sum_{j=1}^{k-2}(-1)^{j-1}\left(1_{j} \otimes t \otimes 1_{k-j-1}\right), \\
\tilde{\partial}^{0}\left(s \cdot 1_{k}\right) & :=\sum_{j=1}^{k-2}\left(1_{j} \otimes(s t-t s) \otimes 1_{k-j-1}\right), \\
\tilde{\partial}^{1}\left(1_{k}\right) & :=0, \quad \tilde{\partial}^{1}\left(s \cdot 1_{k}\right):=t \otimes 1_{k-1}+(-1)^{k} 1_{k-1} \otimes t .
\end{aligned}
$$

Now, let $\tilde{d}$ stand for one of the three symbols $\tilde{d}_{s}, \tilde{\partial}^{0}, \tilde{\partial}^{1}$. Then, using the above formulas, for every $m \geq 1$ and $h_{0}, \ldots, h_{n} \in\left\{1_{k}, s \cdot 1_{k} \mid k \geq 1\right\}$, and $i_{1}, \ldots, i_{n} \geq 1$, we define the corresponding differential by the formula

$$
\tilde{d}\left(h_{0} t^{i_{1}} h_{1} \cdots t^{i_{n}} h_{n}\right)=\sum_{j=0}^{n}(-1)^{\left|h_{0} t^{i_{1}} h_{1} \cdots t^{i_{j-1}} h_{j-1}\right|} \cdot h_{0} t^{i_{1}} h_{1} \cdots t^{i_{j-1}} h_{j-1} t^{i_{j}} \tilde{d}\left(h_{j}\right) t^{i_{j+1}} \cdots t^{i_{n}} h_{n} .
$$

The meaning of the above differentials is explained by Lemma 6.3.1 below (which we will not actually need, but we will need the generalization in Lemma 6.8.1 below). This says that the differentials $\tilde{d}_{s}$ and $\tilde{\partial}^{0}+\tilde{\partial}^{1}$ may be obtained by transporting the natural differentials $\mathrm{d}$ and $\mathbf{i}_{t}$ by means of an appropriate isomorphism. In more detail, let $A_{\tau}=A_{\tau}^{\prime}=\mathbf{k} \oplus \mathbf{k} \cdot \tau$ be a k-algebra with one generator $\tau$ such that $\tau^{2}=0$.

Lemma 6.3.1. The assignment

$$
\zeta: \quad t \mapsto t, \quad s^{\epsilon} \cdot 1_{\ell} \mapsto \tau^{\epsilon}(\mathrm{d} \tau)^{\ell-\epsilon}:=\tau^{\epsilon} \underbrace{\mathrm{d} \tau \mathrm{d} \tau \ldots \mathrm{d} \tau}_{\ell-\epsilon \text { times }}, \quad \epsilon \in\{0,1\}, \quad \ell=1,2, \ldots
$$

yields an isomorphism of bicomplexes

$$
\zeta:\left(\bigoplus_{\ell \geq 1} M^{\otimes \ell}, d_{s}, \tilde{\partial}^{0}+\tilde{\partial}^{1}\right) \stackrel{\sim}{\rightarrow}\left(\bar{\Omega}_{t}\left(A_{\tau}\right), \mathrm{d}, \mathrm{i}_{t}\right) .
$$

Sketch of Proof. The fact that the assignment of the lemma respects the differentials can be verified directly. Further, isomorphism (2.1.1) shows that the set of elements of the form $\tau^{\epsilon}(\mathrm{d} \tau)^{\ell-\epsilon}$ is a $\mathrm{k}$-basis of $\bar{\Omega}\left(A_{\tau}\right)$. Hence, this set combined with the set of words in the elements of the form 
$t^{i} \tau^{\epsilon}(\mathrm{d} \tau)^{\ell-\epsilon}, i \geq 1$, gives a $\mathbf{k}$-basis of $\bar{\Omega}_{t}\left(A_{\tau}\right)$. It follows easily that the map $\zeta$ is a bijection. We note that the direct summand $M^{\otimes \ell}$ goes, via the bijection, to the subspace $\left[\bar{\Omega}_{t}\left(A_{\tau}\right)\right]_{(\ell)}$ of $\bar{\Omega}_{t}\left(A_{\tau}\right)$ spanned by the words in which $\tau$ appears $\ell$ times.

6.4. We will need to compute the homology of the complex $\left(M^{\otimes \ell}, \tilde{\partial}^{0}\right)$.

Lemma 6.4.1. A basis of $H\left(M^{\otimes \ell}, \tilde{\partial}^{0}\right)$ as a free $\mathbf{k}$-module is given by elements of the form

$$
s^{\epsilon_{0}} \otimes(s t+t s)^{\otimes j_{0}} \otimes t^{i_{1}} s^{\epsilon_{1}} \otimes(s t+t s)^{\otimes j_{1}} \otimes \cdots \otimes t^{i_{k}} s^{\epsilon_{k}} \otimes(s t+t s)^{\otimes j_{k}},
$$

for $\epsilon_{0}, \ldots, \epsilon_{k} \in\{0,1\}, j_{0}, \ldots, j_{k} \geq 0$, and $i_{1}, \ldots, i_{k} \geq 2$.

Proof. We define an operation $\tilde{D}$ on $M^{\otimes \ell}$ resembling a derivation which takes $s t$ and $t s$ to $\frac{1}{2} s, t$ to 1, and everything else to zero (such a map cannot actually be a derivation, because the Leibniz rule on $s t$ is not satisfied). Precisely, we define

$$
\tilde{D}\left(t^{i}\right)=\delta_{i, 1}, \quad \tilde{D}\left(s t^{i}\right)=\frac{1}{2} \delta_{i, 1} s=\tilde{D}\left(t^{i} s\right), \quad \tilde{D}\left(s t^{i} s\right)=0,
$$

then write

$$
\tilde{D}\left(1_{p} \otimes f \otimes 1_{q}\right)=(-1)^{p} \cdot 1_{p} \otimes \tilde{D}(f) \otimes 1_{q}
$$

and finally put

$$
\tilde{D}\left(f_{0} t^{i_{1}} f_{1} \cdots t^{i_{k}} f_{k}\right)=\tilde{D}\left(f_{0} t^{i_{1}} f_{1}\right) t^{i_{2}} f_{2} \cdots t^{i_{k}} f_{k}+(-1)^{\mid f_{0} t^{i_{1} \mid}} f_{0} t^{i_{1}} \tilde{D}\left(f_{1} t^{i_{1}} f_{2} \cdots t^{i_{k}} f_{k}\right),
$$

where $f_{0}, \ldots, f_{k} \in T_{S}\langle 1, s\rangle \subseteq T_{S} M$ (i.e., each of these elements can be taken to be either $1_{j}$ for some $j$, or $s \cdot 1_{j}$ for some $j$ ).

Note that both $\tilde{D}$ and $\tilde{\partial}^{0}$ preserve the subcomplexes which are homogeneous of fixed degrees $\geq 2$ in $t$ in specified tensor components: precisely, such a subcomplex is one where, in components $1 \leq c_{1}<\cdots<c_{p} \leq \ell, t$ appears with degrees $d_{1}, \ldots, d_{p} \geq 2$, and $t$ appears with degree $\leq 1$ in all other components.

We are going to show that the operator $D_{[]}:=\left[\tilde{D}, \tilde{\partial}^{0}\right]$ is close to an isomorphism, and that its kernel includes quasi-isomorphically into $\left(M^{\otimes \ell}, \tilde{\partial}^{0}\right)$. To this end, we will compute $D_{[1}$ explicitly.

By the observation from two paragraphs above, this computation reduces to the subspace $\langle 1, t, s t, t s\rangle^{\otimes m}$. More precisely, $D_{[]}$satisfies the following rule which resembles the derivation condition:

$$
D_{[]}\left(g_{1} \otimes t^{i} \otimes g_{2}\right)=D_{[]}\left(g_{1}\right) \otimes t^{i} \otimes g_{2}+g_{1} \otimes t^{i} \otimes D_{[]}\left(g_{2}\right), \quad \forall i \geq 2, g_{1}, g_{2} \in T_{S} M
$$

Moreover, it satisfies another rule which resembles an order-two differential operator condition:

$$
\begin{aligned}
D_{[]}\left(g_{1} \otimes t \otimes f \otimes t \otimes g_{2}\right)=D_{[]}\left(g_{1} \otimes\right. & t \otimes f) \otimes t \otimes g_{2} \\
& +g_{1} \otimes t \otimes D_{[]}\left(f \otimes t \otimes g_{2}\right)-g_{1} \otimes t \otimes D_{[]}(f) \otimes t \otimes g_{2} .
\end{aligned}
$$


This reduces the computation to the six equations

(1) $D_{[]}\left(1_{m}\right)=m \cdot 1_{m}, \quad$ (2) $D_{[]}\left(s \cdot 1_{m}\right)=m s \cdot 1_{m}$,

(3) $D_{[]}\left(1_{m} \otimes t \otimes 1_{n}\right)=(m+n+1)\left(1_{m} \otimes t \otimes 1_{n}\right)$,

(4) $D_{[]}\left(1_{m} \otimes s t \otimes 1_{n}\right)=\left(m+n+\frac{1}{2}\right) 1_{m} \otimes s t \otimes 1_{n}-\frac{1}{2} \sum_{j=0}^{n} 1_{m+j} \otimes t s \otimes 1_{n-j}$,

(5) $D_{[]}\left(1_{m} \otimes t s \otimes 1_{n}\right)=\left(m+n+\frac{1}{2}\right) \cdot 1_{m} \otimes t s \otimes 1_{n}-\frac{1}{2} \sum_{j=0}^{m} 1_{m-j} \otimes s t \otimes 1_{n+j}$,

(6) $\quad D_{[]}\left(s \cdot 1_{m} \otimes t \otimes s \cdot 1_{n}\right)=(m+n) s \cdot 1_{m} \otimes t \otimes s \cdot 1_{n}-\frac{1}{2} \sum_{\substack{0 \leq j \leq m+n \\ j \neq m}} s \cdot 1_{j} \otimes t \otimes s \cdot 1_{m+n-j}$.

Next, we will show that $D_{[]}$is positive and use this to compute its kernel. More precisely, let us assume without loss of generality that $\mathbf{k}=\mathbb{Q}$ (note that $T_{S} M$ is the tensor product of its $\mathbb{Q}$-form by $\mathbf{k}$ ). We equip $T_{S} M$ with the inner product induced by the monomial basis

$$
s^{\epsilon_{0}} \cdot 1_{j_{0}} \otimes t^{i_{1}} s^{\epsilon_{1}} \otimes 1_{j_{1}} \otimes \cdots \otimes t^{i_{r}} s^{\epsilon_{r}} \otimes 1_{j_{r}},
$$

for all $r \geq 0$ and $\epsilon_{0}, \ldots, \epsilon_{r} \in\{0,1\}, j_{0}, \ldots, j_{r} \geq 0$, and $i_{1}, \ldots, i_{r} \geq 1$, i.e., the unique inner product for which this basis is orthonormal.

Next, for each $k \geq 0$, we fix $i_{1}, \ldots, i_{k} \geq 2, j_{0}, \ldots, j_{k} \geq 0$, and $p_{0}, \ldots, p_{k} \geq 0$ such that $p_{r} \leq j_{r}$ for all $r$, and $q_{0}, \ldots, q_{k} \geq 0$ such that $q_{r} \leq p_{r}+1$ for all $r$. Then we define the $D_{[]}$-invariant subspace,

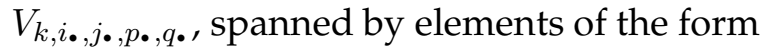

$$
f_{0} \otimes t^{i_{1}} \otimes f_{1} \otimes \cdots \otimes t^{i_{k}} \otimes f_{k},
$$

where, for all $r \in\{0, \ldots, k\}, f_{r} \in T_{S}^{j_{r}}\langle 1, t, s t, t s\rangle$, and it is homogeneous in $s$ and $t$ of degrees $q_{r}$ and $p_{r}$, respectively.

Claim 6.4.7. (a) The operator $D_{[]}$is positive with kernel of dimension $\leq 1$ on $V_{k, i_{\bullet}, j_{\bullet}, p_{\bullet}, q_{\bullet}}$.

(b) This kernel is nonzero if and only if $p_{r}=j_{r}$ and $q_{r} \in\left\{p_{r}, p_{r}+1\right\}$, for all $r$. In this case it is spanned by the element (6.4.2) for $\epsilon_{r}=q_{r}-p_{r}$ where $r=0,1, \ldots, k$.

Proof. In view of Lemma 6.2.1 and (6.4.3), it suffices to prove (a) and (b) in the case that $k=0$. We then denote for simplicity $j=j_{0}, p=p_{0}$, and $q=q_{0}$. The fact that $D_{[]}$is self-adjoint here is immediate from (6.4.4) and (6.4.5)(1)-(6). To show that it is positive, observe again from (6.4.4)(6.4.5) (5) that the symmetric matrix $B=\left(b_{i j}\right)$ for $D_{[]}$on $V_{0, i \bullet, j, p, q}$ in the monomial basis of (6.4.6) satisfies $b_{i i} \geq \sum_{j \neq i}\left|b_{i j}\right|$ for all $i$. Equality holds for all $i$ if and only if the condition of part (b) is satisfied: $p=j$ and $q \in\{p, p+1\}$, i.e., in those formulas (6.4.5)(3)-(6) which arise, always $m=n=0$, and also (6.4.5)(2) does not arise (and neither (6.4.5)(1)).

Observe that the (undirected) graph obtained from $B$, with vertex set $1, \ldots, \operatorname{dim} V_{0, i \cdot j, p, q}$ and with edges between $i$ and $j$ if $b_{i j} \neq 0$, is connected. The claim then follows from Lemma 6.2.2.

Now, we complete the proof of Lemma 6.4.1. We showed that $D_{[]}$is positive with kernel spanned by the elements (6.4.2). By Lemma 6.2.4 this implies that $\left(T_{S} M, \tilde{\partial}^{0}\right)$ is quasi-isomorphic to the subcomplex spanned by the given elements. It is easy to check that $\tilde{\partial}^{0}$ has zero differential restricted to this subcomplex, and that the elements appearing in (6.4.2) are linearly independent. 
6.5. The complex $C_{\ell}$. Write $[s, m]:=s \cdot m-(-1)^{|m|} m \cdot R s$, for $s \in S, m \in M^{\otimes \ell}$. We keep the notation $\otimes=\otimes_{S}$ and put $C_{\ell}:=M^{\otimes \ell} /\left[S, M^{\otimes \ell}\right]$. We introduce a triple $d_{s}, \partial^{0}$, and $\partial^{1}$, of differentials on $C_{\ell}$, as follows.

The definition of the differentials breaks up into two cases: the case of degree zero in $t$ and the case of positive degree in $t$, respectively. In the case of degree zero in $t$, we use the formulas

$$
\begin{array}{ll}
d_{s}\left(1_{\ell}\right)=s \cdot 1_{\ell}, & d_{s}\left(s \cdot 1_{\ell}\right)=0, \\
\partial^{0}\left(1_{\ell}\right)=0, & \partial^{0}\left(s \cdot 1_{\ell}\right)=0, \\
\partial^{1}\left(1_{\ell}\right)=0, & \partial^{1}\left(s \cdot 1_{\ell}\right)=\left(1+(-1)^{\ell}\right) \cdot \sum_{0 \leq j \leq \ell-1} 1_{j} \cdot t \cdot 1_{\ell-j} .
\end{array}
$$

In the case of positive degree in $t$, we observe that the differentials $\tilde{d}_{s}, \tilde{\partial}^{0}$ and $\tilde{\partial}^{1}$ on $M^{\otimes \ell}$, introduced in the previous section, descend to well defined differentials $d_{s}, \partial^{0}$, and $\partial^{1}$, respectively, modulo commutators with $s$. This gives the required differentials on $C_{\ell}=M^{\otimes \ell} /\left[S, M^{\otimes \ell}\right]$.

Let the cyclic group $\mathbb{Z} / \ell$ act on $C_{\ell}$ by cyclic permutations of tensor components using the Koszul sign rule, in terms of the $\mathbb{Z} / 2$ grading above. We write $\left(C_{\ell}\right)_{\mathbb{Z} / \ell}$ for the corresponding space of $\mathbb{Z} / \ell$ coinvariants.

The next lemma is a 'cyclic counterpart' of Lemma 6.3.1 (which we will also not actually need).

Lemma 6.5.1. The map $\zeta$, of Lemma 6.3.1 descends to an isomorphism of bicomplexes

$$
\zeta_{\natural}:\left(\bigoplus_{\ell \geq 1}\left(C_{\ell}\right)_{\mathbb{Z} / \ell}, d_{s}, \partial^{0}+\partial^{1}\right) \stackrel{\sim}{\rightarrow}\left(\overline{\mathrm{DR}}_{t} A_{\tau}, \mathrm{d}, \mathrm{i}_{t}\right) .
$$

Sketch of Proof. The argument is based on the following general result. Let $A$ be an arbitrary algebra, let $V \subset A$ be a k-submodule, and consider $\alpha=a_{0} \mathrm{~d} a_{1} \ldots \mathrm{d} a_{m}$ and $\alpha^{\prime}=a_{0}^{\prime} \mathrm{d} a_{1}^{\prime} \ldots \mathrm{d} a_{n}^{\prime}$ for arbitrary elements $a_{0}, a_{1}, \ldots, a_{m}, a_{0}^{\prime}, a_{1}^{\prime}, \ldots, a_{n}^{\prime} \in V$. Then, the following implication holds:

$$
V \cdot V=0 \Rightarrow\left((\mathrm{d} \alpha) \cdot t \cdot \beta \cdot t \cdot \alpha^{\prime}+(-1)^{|\alpha|+|\beta|} \alpha \cdot t \cdot \beta \cdot t \cdot\left(\mathrm{d} \alpha^{\prime}\right)\right)_{\natural}=0, \quad \forall \beta \in \bar{\Omega}_{t} A .
$$

Now, equation $V \cdot V=0$ obviously holds in the case where $A=A_{\tau}$ and $V=\mathbf{k} \cdot \tau$. Further, it is straightforward to verify that the map $\zeta$ sends the k-submodule $\bigoplus_{\ell}\left[S, M^{\otimes \ell}\right]$, of $\bigoplus_{\ell} M^{\otimes \ell}$, onto the k-submodule of $\bar{\Omega}_{t}\left(A_{\tau}\right)$ spanned by the elements of the form $(\mathrm{d} \alpha) \cdot t \cdot \beta \cdot t \cdot \alpha^{\prime}+(-1)^{|\alpha|+|\beta|} \alpha \cdot t \cdot \beta \cdot t \cdot\left(\mathrm{d} \alpha^{\prime}\right)$, which appear in (6.5.2).

Using this, and the cyclic symmetry of $\overline{\mathrm{DR}}_{t} A_{\tau}$, one shows that the map $\zeta_{\natural}$ is well-defined. To see it is a bijection, we first observe that $\overline{\mathrm{DR}}_{t} A_{\tau}$ can be presented as the quotient of $\bar{\Omega}_{t} A_{\tau}$ by the above relation, for $V=\mathbf{k} \cdot \tau$, along with the relation imposing cyclic symmetry. Then, the result follows from the above observations and the fact that $\zeta$ is a bijection.

6.6. We now compute the homology of the complexes $\left(C_{\ell}, \partial^{0}\right)$ and $\left(C_{\ell} / d_{s} C_{\ell}, \partial^{0}\right)$.

Lemma 6.6.1. (i) A basis for $H\left(C_{\ell}, \partial^{0}\right)$ as a free $\mathbf{k}$-module consists of elements of the form

$$
(s t+t s)^{\otimes j_{0}} \otimes t^{i_{1}} s^{\epsilon_{1}} \otimes(s t+t s)^{\otimes j_{1}} \otimes \cdots \otimes t^{i_{k}} s^{\epsilon_{k}} \otimes(s t+t s)^{\otimes j_{k}},
$$

where $i_{1}, \ldots, i_{k} \geq 2, j_{0}, \ldots, j_{k} \geq 0$, and $\epsilon_{1}, \ldots, \epsilon_{k} \in\{0,1\}$, together with the elements, for all $k \in$ $\{0, \ldots, \ell-1\}$, and $\epsilon \in\{0,1\}$,

$$
\sum_{\substack{1 \leq r \leq k ; j_{0}, \ldots, j_{k} \geq 0: \\ j_{0}+\cdots+j_{k}=\ell-k}}(-1)^{(\epsilon-1)\left(j_{0}+j_{1}+\cdots+j_{r-1}+r-1\right)} \cdot 1_{j_{0}} \otimes t s \otimes \cdots \otimes 1_{j_{r-1}} \otimes t s^{\epsilon} \otimes \cdots \otimes 1_{j_{k-1}} \otimes t s \otimes 1_{j_{k}} .
$$

(ii) A basis for $H\left(C_{\ell} / d_{s} C_{\ell}, \partial^{0}\right)$ as a free $\mathbf{k}$-module consists of elements of the form

$$
(s t+t s)^{\otimes j_{0}} \otimes t \otimes(s t+t s)^{\otimes j_{1}} \otimes t s^{\epsilon_{2}} \otimes(s t+t s)^{\otimes j_{2}} \otimes \cdots \otimes t s^{\epsilon_{k}} \otimes(s t+t s)^{\otimes j_{k}},
$$


where $i_{1}, \ldots, i_{k} \geq 2, j_{0}, \ldots, j_{k} \geq 0$, and $\epsilon_{2}, \ldots, \epsilon_{k} \in\{0,1\}$, together with, for all $k \in\{0, \ldots, \ell-1\}$, the element

$$
\sum_{\substack{1 \leq r \leq k ; j_{0}, \ldots, j_{k} \geq 0: \\ j_{0}+\cdots+j_{k}=\ell-k}}(-1)^{j_{0}+j_{1}+\cdots+j_{r-1}+r-1} \cdot 1_{j_{0}} \otimes t s \otimes \cdots \otimes 1_{j_{r-1}} \otimes t \otimes \cdots \otimes 1_{j_{k-1}} \otimes t s \otimes 1_{j_{k}} .
$$

See the next subsection for an explanation of formulas (6.6.3) and (6.6.5) (they will appear as the associated graded expressions of (6.7.1) with respect to the increasing filtration by the degree in $s$, i.e., the number of times $s$ appears).

Proof. To prove part (i), we will use the proof of Lemma 6.4.1. The key step is to define a homotopy $D$ on $C_{\ell}$ similar to the operator $\tilde{D}$. As before, assume $f_{1}, \ldots, f_{k} \in T_{S}\langle 1, s\rangle \subseteq T_{S} M$. Then, for $i_{1}, \ldots, i_{k} \geq 1$ and $p_{1}, p_{2} \geq 0$, define

$$
\begin{gathered}
D\left(1_{p_{1}} \otimes s^{\epsilon} t^{i_{1}} f_{1} \cdots t^{i_{k-1}} f_{k-1} t^{i_{k}} \otimes 1_{p_{2}}\right)=\tilde{D}\left(1_{p_{1}} \otimes s^{\epsilon} t^{i_{1}} f_{1}\right) t^{i_{2}} f_{2} \cdots t^{i_{k-1}} f_{k-1} t^{i_{k}} \otimes 1_{p_{2}} \\
\left.+(-1)^{\epsilon \cdot \operatorname{deg}\left(t^{i_{1}} f_{1} \cdots t^{i_{k-1}} f_{k-1} t^{i_{k} \otimes 1_{p_{2}}}\right) \cdot 1_{p_{1}} \otimes t^{i_{1}} \tilde{D}\left(f_{1} \cdots t^{i_{k-1}} f_{k-1} t^{i_{k}} \otimes 1_{p_{2}} \cdot R\right.} s^{\epsilon}\right),
\end{gathered}
$$

and so that $D$ kills elements of degree zero in $t$ (elements of the form $\left(f_{1}\right)_{\natural}$ with $f_{1}$ as above). By definition, $D$ commutes with the $\mathbb{Z} / \ell$ action.

By the same argument as before, we can assume $\mathbf{k}=\mathbb{Q}$, and then $\left[D, \partial^{0}\right]$ is a positive operator on $C_{\ell}$, viewed as an inner product space with orthonormal basis

$$
1_{j_{0}} \otimes t^{i_{1}} s^{\epsilon_{1}} \otimes 1_{j_{2}} \otimes \cdots \otimes t^{i_{k}} s^{\epsilon_{k}} \otimes 1_{j_{k}},
$$

for $\epsilon_{1}, \ldots, \epsilon_{k} \in\{0,1\}, j_{0}, \ldots, j_{k} \geq 0$, and $i_{1}, \ldots, i_{k} \geq 1$. The only difference comes in computing the kernel, i.e., the statement of Claim 6.4.7(b) has to be modified (with the same proof, i.e., still using Lemma 6.2.2). Here, we just have to add also the case where $k=0, p_{0}$ is arbitrary, and $q_{0} \in\left\{p_{0}, p_{0}+1\right\}$. One can verify that kernel in this case is spanned by the element (6.6.3) (note that in this formula, there is a different parameter $k$, which need not be zero).

For part (ii), note that $D$ commutes with $d_{s}$, so it descends to an operator on $C_{\ell} / d_{s} C_{\ell}$, which we also denote (abusively) by $D$. Since $\left[D, \partial^{0}\right]$ is positive on $C_{\ell}$ and preserves $d_{s} C_{\ell}$, it is positive on $\left(d_{s} C_{\ell}\right)^{\perp}$. Now, consider the composition $\left(d_{s} C_{\ell}\right)^{\perp} \hookrightarrow C_{\ell} \rightarrow C_{\ell} / d_{s} C_{\ell}$, which is an isomorphism. We claim that it commutes with the actions of $\left[D, \partial^{0}\right]$. This will imply that $\left[D, \partial^{0}\right]$ is also positive on $C_{\ell} / d_{s} C_{\ell}$. The only subtlety here is that $\left(d_{s} C_{\ell}\right)^{\perp}$ is not preserved by $D$ and $\partial^{0}$. However, by definition, the operators $\partial^{0}$ and $D$ on $C_{\ell} / d_{s} C_{\ell}$ are obtained from the projection $C_{\ell} \rightarrow C_{\ell} / d_{s} C_{\ell}$, so the claim follows.

As a result, $\left(C_{\ell} / d_{s} C_{\ell}, \partial^{0}\right)$ is quasi-isomorphic to the subcomplex spanned by the projection of the subcomplex appearing in part (b) to $C_{\ell} / d_{s} C_{\ell}$. This is evidently spanned by the elements (6.6.4)-(6.6.5), since this is the collection of elements from (6.6.2)-(6.6.3) which do not project to zero, and it is easy to see that these remaining elements (6.6.4)-(6.6.5) are linearly independent. As before, the differential $\partial^{0}$ is zero on this subcomplex, so the result follows.

6.7. We continue to keep the notation $\otimes=\otimes_{S}$. For each $\ell \geq 1, q \geq 0$, and $\epsilon \in\{0,1\}$, we consider the image in $C_{\ell}$ of the element

$$
\Upsilon_{q, \ell}^{\epsilon}:=\sum_{\substack{k \geq 0,1 \leq r \leq k, i_{1}, \ldots, i_{k} \geq 1, j_{0}, \ldots, j_{k} \geq 0: \\ i_{1}+\cdots+i_{k}=q, j_{0}+\cdots+j_{k}=\ell-k}}(-1)^{\nu_{\epsilon}\left(j_{0}, \ldots, j_{r-1}\right)} \cdot 1_{j_{0}} \otimes t^{i_{1}} s \otimes \ldots \otimes 1_{j_{r-1}} \otimes t^{i_{r}} s \otimes \cdots \otimes 1_{j_{k-1}} \otimes t^{i_{k}} s \otimes 1_{j_{k}},
$$

where $\nu_{\epsilon}\left(j_{0}, \ldots, j_{r-1}\right):=(\epsilon-1)\left(j_{0}+\cdots+j_{r-1}+r-1\right)$.

Lemma 6.7.2. (1) For all $q \geq 0$ and all $\epsilon \in\{0,1\}$, we have that $\left(\partial^{0}+\partial^{1}\right) \Upsilon_{q, \ell}^{\epsilon}=0$. 
(2) Using the notation of Lemma 6.6.1 one has

(i) The homology $H\left(C_{\ell}, \partial^{0}+\partial^{1}\right)$ is spanned by $\Upsilon_{q, \ell}^{\epsilon}, q \geq 0, \epsilon \in\{0,1\}$.

(ii) The homology $H\left(C_{\ell} / d_{s} C_{\ell}, \partial^{0}+\partial^{1}\right)$ is spanned by $\Upsilon_{q, \ell}^{0}, q \geq 0$.

Proof. Part (1) is an explicit (and straightforward) computation, which we omit.

To prove (2), we introduce an ascending filtration on $C_{\ell}$ by the degree in $s$ (a nonnegative integer). This descends to a filtration on $C_{\ell} / d_{s} C_{\ell}$ as well. The differentials $\partial^{0}$ and $\partial^{1}$ respect the filtration. The associated graded complexes are

$$
\begin{aligned}
\operatorname{gr}_{s}\left(C_{\ell}, \partial^{0}+\partial^{1}\right) & =\left(\operatorname{gr}_{s} C_{\ell}, \partial^{0}\right) \cong\left(C_{\ell}, \partial^{0}\right), \\
\operatorname{gr}_{s}\left(C_{\ell} / d_{s} C_{\ell}, \partial^{0}+\partial^{1}\right) & =\left(\operatorname{gr}_{s}\left(C_{\ell} / d_{s} C_{\ell}\right), \partial^{0}\right) \cong\left(C_{\ell} / d_{s} C_{\ell}, \partial^{0}\right) .
\end{aligned}
$$

One has the standard spectral sequence associated with the filtered complex $\left(C_{\ell}, \partial^{0}+\partial^{1}\right)$. From the isomorphism in (6.7.3), we see that the first page of the spectral sequence is given by the homology of the complex $\left(C_{\ell} / d_{s} C_{\ell}, \partial^{0}\right)$. This homology is described by Lemma 6.7.2. The second page is obtained from the first page by taking the homology of the differential induced by $\partial^{1}$.

We now prove (i); the proof of (ii) is similar. Since $\partial^{0}$ and $\partial^{1}$ are homogeneous of degree one in $t$, we can prove the statement in each fixed degree $q$ in $t$. Let $C_{\ell, q} \subset C_{\ell}$ be the subset of elements of degree $q$ in $t$. The proof rests on comparing $C_{\ell, q}$ with the part of the complex $\left(H\left(\operatorname{gr}_{s} C_{\ell}, \partial^{0}\right), \partial^{1}\right)$ in degree $q+\ell$.

Let $\left(T_{S}^{\ell} M\right)_{q}$ denote the part of $T_{S}^{\ell} M$ of degree $q$ in $t$, and $\left(T_{S}^{\ell} M\right)_{q}^{\prime}$ the subspace spanned by (6.4.2). Let $C_{\ell, q}^{\prime} \subseteq H\left(\operatorname{gr}_{s} C_{\ell}, \partial^{0}\right)$ be the subspace of degree $q$ in $t$, which is spanned by the elements (6.6.2)-(6.6.3). Let $C_{\ell}^{\prime}=\bigoplus_{q \geq 0} C_{\ell, q}^{\prime}$. The differential $\partial^{\prime}$ on $C_{\ell}^{\prime}$, in terms of the basis (6.6.2)-(6.6.3), is given by $\partial^{\prime}=p r \circ \partial^{1}$, where $\partial^{1}$ is given by the usual formula on $C_{\ell}$, and $p r: C_{\ell} \rightarrow C_{\ell}^{\prime}$ is the orthogonal projection in the basis (6.6.6): more precisely, one first projects to $\operatorname{ker}\left(\partial^{0}\right)$ orthogonally and then takes the quotient by $\operatorname{im}\left(\partial^{0}\right)$; equivalently, one projects orthogonally to the span of the elements (6.6.2) - 6.6.3). The reason why this is the differential is that $C_{\ell}^{\prime}$ is obtained from $C_{\ell}$ as the kernel of the positive operator $\left[D, \partial^{0}\right]$, and so the formula for $\partial^{\prime}$ follows from the proof of Lemma 6.2.4 (namely, from the fact that im $\left(\left[D, \partial^{0}\right]\right)$, the orthogonal complement to the span of (6.6.2) - (6.6.3), is an acyclic direct summand of the complex $\left.\left(C_{\ell}, \partial^{0}\right)\right)$.

A key step is to construct a map $\psi_{\ell}$, essentially multiplication by $t^{\otimes \ell}$ with some constants, from $C_{\ell, q}$ to $C_{\ell, q+\ell}^{\prime}$, which commutes with the differential $d_{s}$, and sends $\partial^{0}+\partial^{1}$ to $\partial^{\prime}$. The precise definition of $\psi_{\ell}$ is given on (6.4.6) by

$$
\begin{aligned}
& \psi_{\ell}\left(s^{\epsilon_{0}} \cdot 1_{j_{0}} \otimes t^{i_{1}} s^{\epsilon_{1}} \otimes 1_{j_{1}} \otimes \cdots \otimes t^{i_{k}} s^{\epsilon_{k}} \otimes 1_{j_{k}}\right) \\
&=\left(\left(j_{0}+1\right)^{-1} s\right)^{\epsilon_{0}} \cdot(s t+t s)^{\otimes j_{0}} \otimes t^{i_{1}+1}\left(\left(j_{1}+1\right)^{-1} s\right)^{\epsilon_{1}} \otimes(s t+t s)^{\otimes j_{1}} \otimes \\
& \cdots \otimes t^{i_{k}+1}\left(\left(j_{k}+1\right)^{-1} s\right)^{\epsilon_{k}} \otimes(s t+t s)^{\otimes j_{k}} .
\end{aligned}
$$

Claim 6.7.5. The map $\psi_{\ell}$ is an isomorphism $C_{\ell, q} \rightarrow C_{\ell, q+\ell}^{\prime}$ for all $q \geq 0$ which intertwines $d_{s}$ with $d_{s}$ and intertwines $\partial^{0}+\partial^{1}$ on $C_{\ell}$ with $\partial^{\prime}$ on $C_{\ell}^{\prime}$.

To prove the claim, one has to verify that $\psi_{\ell}$ is an isomorphism, that it preserves $d_{s}$, and that it sends $\partial^{0}+\partial^{1}$ to $\partial^{\prime}$. These facts all follow from straightforward explicit computations, which we omit.

We now complete the proof of Lemma 6.7.2. We do this by induction on $q$. The base case is where $q<\ell$. In this case, $C_{\ell, q}^{\prime}$ is already spanned by the elements $\operatorname{gr}_{s} \Upsilon_{q, \ell}^{\epsilon}, q \geq 0, \epsilon \in\{0,1\}$, which are exactly (6.6.3) up to nonzero constant factors, and, moreover, these elements are killed by $\partial^{\prime}$.

For the inductive step, assume that, in degree $q, H\left(C_{\ell}, \partial^{0}+\partial^{1}\right)$ is spanned by the elements $\Upsilon_{q, \ell}^{\epsilon}$ for $\epsilon \in\{0,1\}$. By Claim 6.7.5, this implies that, in degree $q+\ell, H\left(C_{\ell}^{\prime}, \partial^{\prime}\right)$ is spanned by $\psi_{\ell}\left(\Upsilon_{q, \ell}^{\epsilon}\right)$. 
We claim that

$$
\psi_{\ell}\left(\Upsilon_{q, \ell}^{\epsilon}\right)=\mathrm{gr}_{s} \Upsilon_{q+\ell, \ell}^{\epsilon} .
$$

Then, by the spectral sequence for $\left(C_{\ell}, \partial^{0}+\partial^{1}\right)$, this implies that the homology of $\left(C_{\ell}, \partial^{0}+\partial^{1}\right)$ in degree $q+\ell$ is spanned by the classes $\Upsilon_{q+\ell, \ell}^{\epsilon}$ completing the induction.

It remains to prove (6.7.6). Clearly, the RHS is given by restricting the sum in (6.7.1) to the summands where $k=\ell$. In this case, each such summand is obtained from the summand of element $\Upsilon_{q, \ell}^{\epsilon}$. This proves (6.7.6).

6.8. Proof of Proposition 6.1.1. The proof amounts to a generalization of the preceding material from $A_{\tau}$ to $A^{\prime}$. Here we revert to the notation $\otimes=\otimes_{\mathbf{k}}$ used before (and as we will use in the remainder of the paper).

Below, we will use, for every $p, q \geq 0$, a direct sum decomposition $\bar{\Omega}_{t}^{p, q} A^{\prime}=\oplus_{\ell \geq 1}\left[\bar{\Omega}_{t}^{p, q} A^{\prime}\right]_{(\ell)}$, where $\left[\bar{\Omega}_{t}^{p, q} A^{\prime}\right]_{(\ell)}$ denotes the span of all elements in which $\bar{A}$ appears $\ell$ times. This induces a similar decomposition $\overline{\mathrm{DR}}_{t}^{p, q} A^{\prime}=\oplus_{\ell \geq 1}\left[\overline{\mathrm{DR}}_{t}^{p, q} A^{\prime}\right]_{(\ell)}$. Also, let a superscript of $q$ denote the degree $q$ part in $t$.

For each $\ell \geq 1$, we define a map $\zeta: \bar{A}^{\otimes \ell} \otimes M^{\otimes_{S} \ell} \longrightarrow\left[\bar{\Omega}_{t} A^{\prime}\right]_{(\ell)}$ as follows. In positive degree in $t$, the map is given, for every $f_{1}, \ldots, f_{\ell} \in\left\{1, t^{i}, t^{i} s \mid i \geq 1\right\} \subset M$ not all equal to 1 , and $a_{1}, \ldots, a_{\ell} \in \bar{A}$, by the formula

$$
\left(a_{1} \otimes \cdots \otimes a_{\ell}\right) \otimes\left(f_{1} \otimes_{S} \cdots \otimes_{S} f_{\ell}\right) \mapsto\left(f_{1} \star a_{1}\right)\left(f_{2} \star a_{2}\right) \cdots\left(f_{\ell} \star a_{\ell}\right),
$$

where we put

$$
1 \star a=\mathrm{d} a, \quad t^{i} \star a=t^{i} a, \quad t^{i} s \star a=t^{i} \mathrm{~d} a, \quad i \geq 1 .
$$

In degree zero in $t$, we use the formulas

$$
\left(a_{1} \otimes \cdots \otimes a_{\ell}\right) \otimes\left(1_{\ell}\right) \mapsto a_{1} \mathrm{~d} a_{2} \cdots \mathrm{d} a_{\ell}, \quad\left(a_{1} \otimes \cdots \otimes a_{\ell}\right) \otimes\left(s \cdot 1_{\ell}\right) \mapsto \mathrm{d} a_{1} \mathrm{~d} a_{2} \cdots \mathrm{d} a_{\ell} .
$$

Recall next that the cyclic group $\mathbb{Z} / \ell$ acts on $C_{\ell}$ by cyclic permutations of tensor components using the Koszul sign rule. We let $\mathbb{Z} / \ell$ act on $\bar{A}^{\otimes \ell}$ by ordinary cyclic permutations and we equip $\bar{A}^{\otimes \ell} \otimes C_{\ell}$ with $\mathbb{Z} / \ell$-diagonal action. Further, we extend each of the three differentials $d=d_{s}, \partial^{0}$, and $\partial^{1}$ on $C_{\ell}$ to the differentials $d_{A}:=\mathrm{Id} \otimes d$ on $\bar{A}^{\otimes \ell} \otimes C_{\ell}$, where Id : $\bar{A}^{\otimes \ell} \rightarrow \bar{A}^{\otimes \ell}$ is the identity. Similarly, we extend the tilde versions $\tilde{d}$ to $\tilde{d}_{A}:=\operatorname{Id} \otimes \tilde{d}$ on $\bar{A}^{\otimes \ell} \otimes M^{\otimes} \ell$. Finally, for every $q \geq 0$, let $\left(\bar{A}^{\otimes \ell} \otimes M^{\otimes S^{\ell}}\right)^{q}$ and $\left(\bar{A}^{\otimes \ell} \otimes C_{\ell}\right)_{\mathbb{Z} / \ell}^{q}$ be the homogeneous component of $\bar{A}^{\otimes \ell} \otimes M^{\otimes_{S} \ell}$ and $\left(\bar{A}^{\otimes \ell} \otimes C_{\ell}\right)_{\mathbb{Z} / \ell}$ of degree $q$ in $t$, respectively.

Lemma 6.8.1. For $\ell \geq 1$, (i) For every $q \geq 0$, the map $\zeta$ induces isomorphisms of complexes

$$
\left(\left(\bar{A}^{\otimes \ell} \otimes M^{\otimes S} \ell\right)^{q}, d_{s, A}\right) \stackrel{\sim}{\rightarrow}\left(\left[\bar{\Omega}_{t}^{\cdot, q} A^{\prime}\right]_{(\ell)}, \mathrm{d}\right), \quad \text { and } \quad\left(\left(\bar{A}^{\otimes \ell} \otimes C_{\ell}\right)_{\mathbb{Z} / \ell}^{q}, d_{s, A}\right) \stackrel{\sim}{\rightarrow}\left(\left[\overline{\mathrm{DR}}_{t}^{\cdot, q} A^{\prime}\right]_{(\ell)}, \mathrm{d}\right) \text {. }
$$

(ii) For $\epsilon \in\{0,1\}$ and $\ell \geq 1$, put $\Upsilon_{\ell}^{\epsilon}:=\sum_{q \geq 0} \frac{1}{(q+\ell-1) !} \cdot \Upsilon_{q, \ell}^{\epsilon}$. Then, for every $\alpha \in \bar{A}^{\otimes \ell}$, the following diagram commutes:

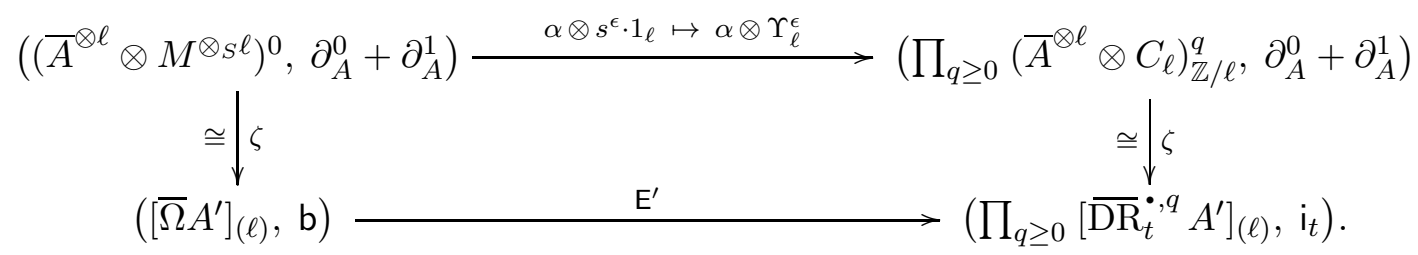


Sketch of proof. In the special case where $\bar{A}=\mathbf{k}$ is a rank one free $\mathbf{k}$-module, $A^{\prime}=A_{\tau}=\mathbf{k} \oplus \mathbf{k} \tau$. In this case, the isomorphisms of part (i) of the lemma, as well as fact that the map $\zeta$ respects the differentials, reduce to Lemmas 6.3.1 and 6.5.1. The proof in the general case is similar. Note that implication (6.5.2), used in the proof of Lemma 6.5.1, is applicable in the general case as well, since in the algebra $A^{\prime}$ it follows that $\bar{A} \cdot \bar{A}=0$.

Finally, commutativity of the diagram of part (ii) is verified by direct computation using the explicit formula for the Tsygan map and for the map $\zeta$. We omit the details.

Remark 6.8.2. In the special case $A=A_{\tau}$, i.e., $\bar{A}=\mathbf{k}$, the commutative diagram of Lemma 6.8.1(ii) reduces to the diagram:

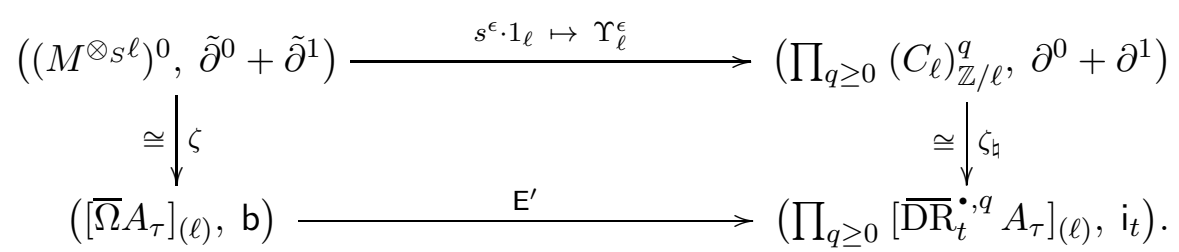

We remark that it is immediate from commutativity of this diagram that $\left(\partial^{0}+\partial^{1}\right) \Upsilon_{\ell}^{\epsilon}$ maps to zero in $\left(C_{\ell}\right)_{\mathbb{Z} / \ell}$. This way, one obtains the equation of Lemma 6.7.2(1), but only modulo cyclic permutations.

Proposition 6.1.1 is a direct consequence of the above lemma and Lemma 6.7.2.

\section{PeRiodic AND NegATIVE EXTENDED CyClic HOMOLOGY}

7.1. We define extended periodic and negative cyclic homology of $A$ as follows

$$
\overline{\mathrm{EHC}}^{\mathrm{per}}(A):=H\left(\overline{\mathrm{DR}}_{t}((u)), \mathrm{i}_{t}-u \mathrm{~d}\right), \quad \text { and } \quad \overline{\mathrm{EHC}}^{-}(A):=H\left(\overline{\mathrm{DR}}_{t} \llbracket u \rrbracket, \mathrm{i}_{t}-u \mathrm{~d}\right) .
$$

Similarly, let us define versions using the $t$-adic completion, $\overline{\mathrm{EHC}}^{\mathrm{per}, \wedge}:=H\left(\overline{\mathrm{DR}}_{\mathrm{t}}^{\wedge}((u)), \mathrm{i}_{t}-u \mathrm{~d}\right)$ and $\overline{\mathrm{EHC}}^{-, \wedge}(A):=H\left(\overline{\mathrm{DR}}_{\mathrm{t}}^{\wedge} \llbracket u \rrbracket, \mathrm{i}_{t}-u \mathrm{~d}\right)$.

Remark 7.1.1. The complexes above can be viewed as bicomplexes with differentials $u \mathrm{~d}$ and $\mathrm{i}_{t}$. Each of the two differentials preserves 'internal degree,' defined as the sum of de Rham degree and degree in $t$ minus degree in $u$. Separating homogeneous components with respect to internal degree gives decompositions of $\left(\overline{\mathrm{DR}}_{\mathrm{t}}^{\wedge}((u)), \mathrm{i}_{t}-u \mathrm{~d}\right)$ and $\left(\overline{\mathrm{DR}}_{\mathrm{t}}^{\wedge} \llbracket u \rrbracket, \mathrm{i}_{t}-u \mathrm{~d}\right)$ into infinite products of subcomplexes. (Unlike for $\left(\overline{\mathrm{DR}}_{t} \otimes R, \mathrm{i}_{t}-u \mathrm{~d}\right)$, the uncompleted versions here are not direct sums of subcomplexes in each internal degree, which is why we restrict our attention in this section to the $t$-adically completed versions, which are better behaved.)

Proposition 7.1.2. The projection $\left(\overline{\mathrm{DR}}_{\mathrm{t}}^{\wedge}((u)), \mathrm{i}_{t}-u \mathrm{~d}\right) \rightarrow(\overline{\mathrm{DR}}((u)),-u \mathrm{~d})$, modulo $t$, is a morphism of complexes, inducing an isomorphism on homology $\overline{\mathrm{EHC}}^{\text {per, } \wedge}(A) \stackrel{\sim}{\rightarrow} \overline{\mathrm{HD}}(A)((u))$.

Remark 7.1.3. The above isomorphism, as well as all other isomorphisms which appear in this section, preserves the 'homological grading,' which is defined to be de Rham degree minus twice the degree in $u$.

Proof of Proposition. Exactly as in the proof of Proposition 5.6.1, we can take homology first with respect to $u \mathrm{~d}$ and use a spectral sequence. This spectral sequence is obviously convergent on each homogeneous component with respect to the internal degree, cf. Remark 7.1.1; moreover, it collapses on the first page (the homology with respect to $u \mathrm{~d}$ ).

Remark 7.1.4. One can similarly compute $\overline{\mathrm{EHC}}^{-, \wedge}(A)$. First of all, observe that a summand of $\operatorname{ker}_{\overline{\mathrm{HH}}(A)}(B \circ I)$ (recall (4.1.3) for the definitions of $B$ and $I$ ) splits off in degree zero in both $t$ and 
$u$. As in the literature, let us make the abuse of notation $B:=B \circ I: \overline{\mathrm{HH}}(A) \rightarrow \overline{\mathrm{HH}}(A)$ for the induced differential on $\overline{\mathrm{HH}}(A)$.

Claim 7.1.5. There is a natural exact sequence

$$
0 \rightarrow \prod_{q \geq 1} \frac{H\left(\overline{\mathrm{DR}}_{t} / \mathrm{d} \overline{\mathrm{DR}}_{t}, \mathrm{i}_{t}\right)^{\bullet, q}}{\left(\tilde{\mathrm{d}}^{-1} \mathrm{i}_{t}\right)^{q}(\overline{\mathrm{HD}}(A))} \stackrel{\mathrm{d}}{\longrightarrow} \frac{\overline{\mathrm{EHC}^{-, \wedge}(A)}}{\operatorname{ker}_{\overline{\mathrm{HH}}(A)}(B)} \stackrel{\pi}{\longrightarrow} \prod_{m \geq 1} u^{m} \cdot \operatorname{ker}_{\overline{\mathrm{HD}}(A)}\left(\left(\tilde{\mathrm{d}}^{-1} \mathrm{i}_{t}\right)^{m+1}\right) \rightarrow 0 .
$$

Here, the map $d$ is induced by the obvious map $\bar{d}: \overline{\mathrm{DR}}_{\mathrm{t}}^{\wedge} / \mathrm{d} \overline{\mathrm{DR}}_{\mathrm{t}}^{\wedge} \rightarrow \overline{\mathrm{DR}}_{\mathrm{t}}^{\wedge}$, and the map $\pi$ mods by the constant term in $u$ and by positive degree in $t$. Further, $\left(\tilde{\mathrm{d}}^{-1} \mathrm{i}_{t}\right)^{q}: \overline{\mathrm{HD}}(A) \rightarrow H\left(\overline{\mathrm{DR}}_{t} / \mathrm{d} \overline{\mathrm{DR}}_{t}, \mathrm{i}_{t}\right)^{\bullet}, q$ is the map considered in $\$ 5.7$ (see the proof of Proposition 5.7.1). More explicitly, the map $\tilde{d}^{-1} \mathrm{i}_{t}$ induces an endomorphism of $H\left(\overline{\mathrm{DR}}_{t} / \mathrm{d} \overline{\mathrm{DR}}_{t}, \mathrm{i}_{t}\right)$ as follows. Given an element of $H\left(\overline{\mathrm{DR}}_{t} / \mathrm{d} \overline{\mathrm{DR}}_{t}, \mathrm{i}_{t}\right)$, one first lifts it to $\overline{\mathrm{DR}}_{t}$, then applies $\mathrm{i}_{t}$. After that, one applies $\tilde{\mathrm{d}}^{-1}$, and projects to $\overline{\mathrm{DR}}_{t} / \mathrm{d} \overline{\mathrm{DR}}_{t}$,

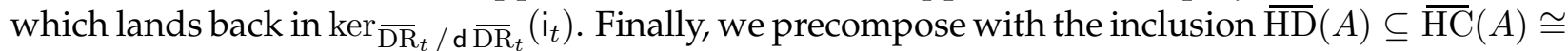
$H\left(\overline{\mathrm{DR}}_{t} / \mathrm{d} \overline{\mathrm{DR}}_{t}, \mathrm{i}_{t}\right)^{\bullet, 0} \subseteq H\left(\overline{\mathrm{DR}}_{t} / \mathrm{d} \overline{\mathrm{DR}}_{t}, \mathrm{i}_{t}\right)$.

By definition, $\left.\left(\tilde{\mathrm{d}}^{-1} \mathrm{i}_{t}\right)^{m}\right|_{\overline{\mathrm{HD}}_{k}(A)}=0$ when $k<2 m$. Thus, for fixed $k$ and $m>k / 2-1$, the component $u^{m} \cdot \operatorname{ker}_{\overline{\mathrm{HD}}_{k}(A)}\left(\left(\tilde{\mathrm{d}}^{-1} \mathrm{i}_{t}\right)^{m+1}\right)$ of the above product is nothing but $u^{m} \cdot \overline{\mathrm{HD}}_{k}(A)$.

Further, using isomorphism (5.5.5), the short exact sequence of Claim 7.1.5 becomes

$$
0 \rightarrow \prod_{q \geq 1} \frac{\overline{\mathrm{HC}}(A) \cdot t^{q}}{\operatorname{im}\left(S^{q+1}\right)} \longrightarrow \frac{\overline{\mathrm{EHC}^{-, \wedge}(A)}}{\operatorname{ker}_{\overline{\mathrm{HH}}(A)}(B)} \rightarrow \prod_{m \geq 1} u^{m} \cdot \operatorname{ker}_{\overline{\mathrm{HD}}(A)}\left(\left(S_{1} S_{2}\right)^{m+1}\right) \rightarrow 0,
$$

where we note that the $q$ became $q+1$ in the first factor since the periodicity operator $S$ is acting on $\overline{\mathrm{HC}}(A)$ rather than on $\overline{\mathrm{HD}}(A)=\operatorname{im}\left(S_{1}\right)$.

Proof of Claim 7.1.5 We use the spectral sequence for the bicomplex $\left(\overline{\mathrm{DR}}_{\mathrm{t}}^{\wedge} \llbracket u \rrbracket, u \mathrm{~d}, \mathrm{i}_{t}\right)$, taking homology first with respect to $u \mathrm{~d}$. This yields the first page,

$$
\left(u \cdot \overline{\operatorname{HD}}(A) \llbracket u \rrbracket \oplus \operatorname{ker}_{\overline{D R}_{\mathrm{t}}^{\wedge}}(\mathrm{d}), 0 \oplus \mathrm{i}_{t}\right) .
$$

Using the isomorphism $\mathrm{d}:\left(\overline{\mathrm{DR}}_{\mathrm{t}}^{\wedge}\right)^{+} / \mathrm{d}\left(\overline{\mathrm{DR}}_{\mathrm{t}}^{\wedge}\right)^{+} \stackrel{\sim}{\rightarrow} \mathrm{d}\left(\overline{\mathrm{DR}}_{\mathrm{t}}^{\wedge}\right)^{+}$, which commutes with $\mathrm{i}_{t}$, the second page becomes

$$
u \cdot \overline{\mathrm{HD}}(A) \llbracket u \rrbracket \oplus H^{>0}\left(\overline{\mathrm{DR}}_{\mathrm{t}}^{\wedge} / \mathrm{d} \overline{\mathrm{DR}}_{\mathrm{t}}^{\wedge}, \mathrm{i}_{t}\right) /\left(\tilde{\mathrm{d}}^{-1} \mathrm{i}_{t} \overline{\mathrm{HD}}(A)\right) \oplus \operatorname{ker}_{\overline{\mathrm{HH}}(A)}(\mathrm{d}) .
$$

The terms on the left and right of the exact sequence of Claim 7.1 .5 then appear on the $(m+2)$-nd and $(q+1)$-st pages. One sees that the spectral sequence converges to this, i.e., there are no other nontrivial differentials that appear. Therefore, the associated graded (where by this we mean an infinite product of homogeneous components) of $\overline{\mathrm{EHC}}^{-, \wedge}(A)$ with respect to the $t$-adic filtration is the above, and one can check that the maps in the exact sequence of Claim 7.1.5induce the required isomorphism on the level of associated graded, so the sequence there must be exact (with injective first arrow and surjective third arrow).

Remark 7.1.7. According to (5.5.2), Proposition 7.1.2, and (7.1.6), the extended versions of all cyclic homology groups $\overline{\mathrm{EHC}}(A), \overline{\mathrm{EHC}}^{\mathrm{per}, \wedge}(A)$, and $\overline{\mathrm{EHC}}^{-, \wedge}(A)$ are expressible in terms of the original Connes sequence (4.1.2), and do not require $\overline{\mathrm{HC}}^{-}(A)$ or $\overline{\mathrm{HC}}^{\mathrm{per}}(A)$, which use infinite series in their definition. In other words, taking the extended version breaks the complex up into a direct product of complexes only involving finite sums, i.e., elements of $\overline{\mathrm{DR}}_{t}\left[u, u^{-1}\right]$ (and via Theorem 5.3.10, elements of $\bar{\Omega}\left[u, u^{-1}\right]$ ), owing to the internal grading. As a consequence, the natural morphisms we will describe in $₫ 8$ from the various flavors of cyclic homology of $A$ to the (equivariant) cohomology of the representation varieties of $A$ all factor through (infinite products of) subquotients of $\overline{\mathrm{HC}}(A)$ and $\overline{\mathrm{HH}}(A)$. 
7.2. The result below describes the maps induced by $\mathrm{E}$ on the versions of cyclic homology.

Let $\pi^{\prime}: \overline{\operatorname{EHC}}(A) \rightarrow \overline{\mathrm{HD}}(A) \otimes u^{-1} \mathbf{k}\left[u^{-1}\right] \subseteq \overline{\mathrm{HD}}(A) \otimes R$ be the map given by $f=\sum_{m>0} f_{m} u^{-m} \mapsto$ $\sum_{m \geq 1} f_{m} u^{-m} \bmod (t)$, cf. Proposition 5.6.1, Let $\overline{\mathrm{EHC}}^{\wedge}(A)=H\left(\overline{\mathrm{DR}}_{\mathrm{t}}^{\wedge} \otimes R, \mathrm{i}_{t}-u \mathrm{~d}\right) \cong \overline{\mathrm{HC}}(A) \llbracket t \rrbracket \oplus$ $u^{-1} \overline{\mathrm{HD}}(A)\left[u^{-1}\right]$, and define $\pi^{\prime}$ in the same way on it.

Proposition 7.2.1. (i) The composite map $\overline{\mathrm{HC}}(A) \stackrel{\mathrm{E}}{\longrightarrow}{\overline{\mathrm{EHC}^{\wedge}}}(A) \stackrel{\pi^{\prime}}{\longrightarrow} \overline{\mathrm{HD}}(A) \otimes R$ can be expressed as $\pi^{\prime} \circ \mathrm{E}=\sum_{m \leq 1} u^{-m} \cdot S_{1} S^{m-1}$.

(ii) The induced map $\overline{\mathrm{HC}}^{\mathrm{per}}(A) \rightarrow \overline{\mathrm{EHC}}^{\mathrm{per}, \wedge}(A) \cong \overline{\mathrm{HD}}(A)((u))$ is $\sum_{m \in \mathbb{Z}} u^{-m} \cdot p_{1} u^{m}$.

(iii) The induced map $\overline{\mathrm{HC}}^{-}(A) \rightarrow \overline{\mathrm{EHC}}^{-, \wedge}(A)$, in terms of (7.1.5), is the sum of $I^{-}$to $\operatorname{ker}_{\overline{\mathrm{HH}}(A)}(\mathrm{d})$ with the map which is $\sum_{m \geq 1} u^{m}\left(p_{1} u^{-m} p^{-}\right)$projected to the subspace of $\prod_{m \geq 1} u^{m} \cdot \operatorname{ker}_{\overline{\mathrm{HD}}(A)}\left(\left(\tilde{\mathrm{d}}^{-1} \mathrm{i}_{t}\right)^{m+1}\right)$, and, restricted to the kernel of this map, maps to the zero fiber $\prod_{q \geq 1} H\left(\overline{\mathrm{DR}}_{t} / \mathrm{d} \overline{\mathrm{DR}}_{t}, \mathrm{i}_{t}\right)^{\bullet, q} / \mathrm{im}\left(\left(\tilde{\mathrm{d}}^{-1} \mathrm{i}_{t}\right)^{q}\right)$ by $\sum_{q \geq 1} \mathrm{E}^{q} \circ p \circ p^{-}$.

The proof is a direct consequence of formula (5.3.9), Propositions 5.6.1 and 7.1.2 and Remark 7.1.4

7.3. Extended version of the Connes exact sequence. There is an extended analogue of diagram (4.4.4). One can obtain this diagram by applying $E$ to the diagram of short exact sequences of complexes that induce (4.4.4) (in the usual proof). This yields

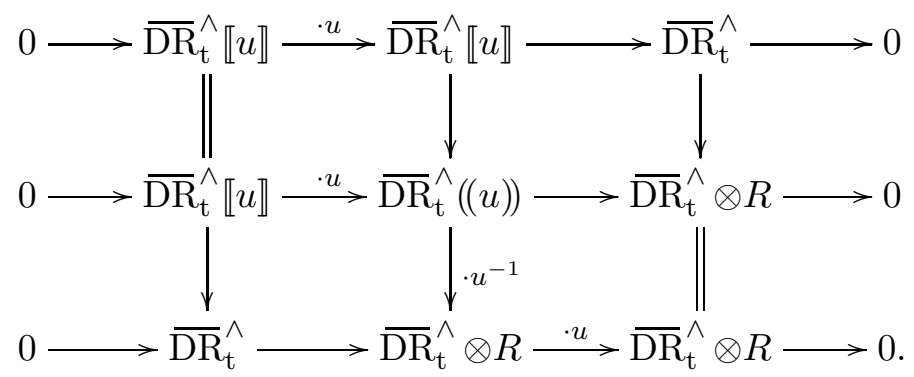

This induces the diagram (4.4.4) of short exact sequences, with $E$ put in front of every nonzero term and $t$-adic completions taken everywhere, where $\overline{\mathrm{EHH}}, \overline{\mathrm{EHH}^{\prime}}, \overline{\mathrm{EHD}}$, and $\overline{\mathrm{EHD}}^{\prime}$ are given by:

$$
\begin{gathered}
\overline{\operatorname{EHH}}(A):=H\left(\overline{\mathrm{DR}}_{t}, \mathrm{i}_{t}\right), \quad \overline{\operatorname{EHH}}^{\prime}(A):=\bigoplus_{q \geq 0}\left[\left(\tilde{\mathrm{d}}^{-1} \mathrm{i}_{t}\right)^{q}\left(\operatorname{ker}_{\overline{\mathrm{DR}}}(\mathrm{d})\right) \cap \operatorname{ker}\left(\mathrm{i}_{t}\right)\right] \subseteq \overline{\operatorname{EHH}}(A), \\
\overline{\operatorname{EHD}}(A):=\overline{\mathrm{HD}}(A)\left[u^{-1}\right] \oplus \bigoplus_{q \geq 1} \operatorname{ker}\left(\mathrm{d}: H\left(\overline{\mathrm{DR}}_{t} / \mathrm{d} \overline{\mathrm{DR}}_{t}, \mathrm{i}_{t}\right) \rightarrow H\left(\overline{\mathrm{DR}}_{t}, \mathrm{i}_{t}\right)\right), \\
\overline{\operatorname{EHD}}^{\prime}(A):=\overline{\operatorname{HD}}(A)\left[u^{-1}\right] \oplus \bigoplus_{q \geq 1}\left(\tilde{\mathrm{d}}^{-1} \mathrm{i}_{t}\right)^{q}(\overline{\mathrm{HD}}(A)) \subseteq \overline{\operatorname{EHD}}(A),
\end{gathered}
$$

and the $t$-adically completed versions are obtained by replacing $\overline{\mathrm{DR}}_{t}$ with $\overline{\mathrm{DR}}_{\mathrm{t}}^{\wedge}$, and the direct sums above by direct products. Then, $\mathrm{E}$ maps (4.4.4) commutatively onto the resulting diagram.

Remark 7.3.1. By Theorem 5.3.10, one has isomorphisms $\mathrm{E}^{q}: \overline{\mathrm{HH}}(A) \stackrel{\sim}{\rightarrow} \overline{\mathrm{EHH}}(A)^{\bullet}, q, q \geq 0$. We deduce that $\overline{\mathrm{EHH}}^{\prime}(A)^{\bullet, 0} \cong \operatorname{ker}_{\overline{\mathrm{HH}}(A)}(B)=I^{-1}(\operatorname{im} S)$, where $S: \overline{\mathrm{HC}}(A) \rightarrow \overline{\mathrm{HC}}(A)$ is the periodicity operator. Then one obtains:

$$
\begin{aligned}
& \overline{\mathrm{EHH}}(A) \cong \overline{\mathrm{HH}}(A)[t] \quad \supseteq \overline{\mathrm{EHH}}^{\prime}(A) \cong \oplus_{q \geq 0} I^{-1}\left(\operatorname{im}\left(S^{q+1}\right)\right) \cdot t^{q}, \\
& \overline{\operatorname{EHD}}(A) \cong \overline{\operatorname{HD}}(A)\left[u^{-1}\right] \bigoplus t \overline{\operatorname{HD}}(A)[t] \supseteq \overline{\operatorname{EHD}}^{\prime}(A) \cong \overline{\operatorname{HD}}(A)\left[u^{-1}\right] \bigoplus\left(\oplus_{q \geq 1} \operatorname{im}\left(S_{1} S_{2}\right)^{q} \cdot t^{q}\right) \text {. }
\end{aligned}
$$


The $t$-adically completed versions of $\overline{\mathrm{EHH}}(A), \overline{\mathrm{EHH}}^{\prime}(A), \overline{\mathrm{EHD}}(A)$, and $\overline{\mathrm{EHD}}^{\prime}(A)$ are all obtained by $t$-adically completing the above formulas (replacing sums by products and polynomials in $t$ by power series in $t$ ).

Remark 7.3.2. One can explicitly compute the extended, $t$-adically completed version of (4.4.4) in terms of the preceding remark along with (5.5.2), Proposition 7.1.2, and (7.1.6), and verify that the rows are exact using only exactness of (4.4.4). We describe just the horizontal maps in these terms:

The bottom row:

$E S_{2}$ : This is the identity on $u^{-1} \overline{\mathrm{HD}}(A)\left[u^{-1}\right]$ and the inclusion $S_{2}$ on $\overline{\mathrm{HD}}(A) \llbracket t \rrbracket \subseteq \overline{\mathrm{HC}}(A) \llbracket t \rrbracket$, preserving degree in $t$.

$E B: \overline{\mathrm{EHC}}^{\wedge}(A) \cong u^{-1} \overline{\mathrm{HD}}(A)\left[u^{-1}\right] \oplus \overline{\mathrm{HC}}(A) \llbracket t \rrbracket \rightarrow \overline{\mathrm{EHH}}^{\wedge}(A) \cong \overline{\mathrm{HH}}(A) \llbracket t \rrbracket$ is zero on the first factor of the source and $B$ on the second factor.

$E I: \overline{\mathrm{EHH}}^{\wedge}(A) \cong \overline{\mathrm{HH}}(A) \llbracket t \rrbracket \rightarrow \overline{\mathrm{EHC}}^{\wedge}(A)=u^{-1} \overline{\mathrm{HD}}(A)\left[u^{-1}\right] \oplus \overline{\mathrm{HC}}(A) \llbracket t \rrbracket$ is zero on the first factor of the target, and $I$ on the second factor of the target.

$E S_{1}$ is the tautological map (preserving the degree in $t$ ).

The middle row:

$E B^{\prime}: \overline{\mathrm{EHC}}^{\wedge}(A) \rightarrow \overline{\mathrm{EHC}}^{-, \wedge}(A)$ : in terms of (7.1.5), this kills $u^{-1} \overline{\mathrm{HD}}\left[u^{-1}\right]$, maps by $B$ on $\overline{\mathrm{HC}}(A)$ in degree $q=0$ of $t$, and for positive degree $q \geq 1$ in $t$, it is the projection $\overline{\mathrm{HC}}(A) \rightarrow \overline{\mathrm{HC}} / \mathrm{im} S^{q+1} \subseteq$ $\overline{\mathrm{EHC}}^{-, \wedge}(A)$ by $(\mathbf{7 . 1 . 5})$.

$E p^{-}: \overline{\operatorname{EHC}}^{-, \wedge}(A) \rightarrow \overline{\operatorname{EHC}}^{p e r, \wedge}(A)$ projects onto $\operatorname{ker}_{\overline{\mathrm{HH}}(A)}(B) \oplus \prod_{m \geq 1} u^{m} \operatorname{ker}_{\overline{\mathrm{HD}}(A)}\left(S_{1} S_{2}\right)^{m+1}$, and then applies $I$ to the first factor and the natural inclusion on the second factor into $u \overline{\operatorname{HD}} \llbracket u \rrbracket$, preserving degree in $u$.

$E p_{1}$ is the identity on $\overline{\mathrm{HD}}(A)\left[u^{-1}\right]$ and sends $u^{m} \overline{\mathrm{HD}}(A)$ to the factor for $q=m$ by the map $S^{m} S_{2} u^{-m} t^{m}$.

$E p_{2}$ is the tautological inclusion (preserving degree in $t$ ).

The first row:

The first map is the tautological inclusion.

$E B^{-}$maps $\overline{\mathrm{HH}}(A)$ via $B$ to $\operatorname{ker}_{\overline{\mathrm{HH}}(A)}(B)$ in degree $q=0$ of $t$, and in higher degrees $q \geq 1$ maps $\overline{\mathrm{HH}}(A)$ to $\overline{\mathrm{HC}}(A)$ via $I$ and projects to $\overline{\mathrm{HC}}(A) / \operatorname{im} S^{q+1}$.

$E S^{-}$applies $t \cdot S$ to $\overline{\mathrm{HC}}(A) t^{q} / \operatorname{im} S^{q+1}$ for $q \geq 1$, increasing degree in $t$ by one. Modulo these factors (the kernel of the sequence (7.1.5)), it multiplies by $u$ : on the quotient of (7.1.5) these are the inclusions $\operatorname{ker}_{\overline{\mathrm{HD}}(A)}\left(S_{1} S_{2}\right)^{m+1} \subseteq \operatorname{ker}_{\overline{\mathrm{HD}}(A)}\left(S_{1} S_{2}\right)^{m+2}$, and on $\operatorname{ker}_{\overline{\mathrm{HH}}(A)}(B)$ this is the natural map $\operatorname{ker}_{\overline{\mathrm{HH}}(A)}(B) \rightarrow \operatorname{ker}_{\overline{\mathrm{HD}}(A)}\left(S_{1} S_{2}\right)$ (this follows because $\operatorname{ker}_{\overline{\mathrm{HH}}(A)}(B)=\operatorname{ker}_{\overline{\mathrm{DR}}}(\mathrm{d}) \cap \operatorname{ker}_{\overline{\mathrm{DR}}}\left(\mathrm{i}_{t}\right)$, so projecting mod d $\overline{\mathrm{DR}}$ we land in $\left.\operatorname{ker}_{\overline{\mathrm{HD}}(A)}\left(S_{1} S_{2}\right) \subseteq \operatorname{ker}_{\overline{\mathrm{HD}}(A)}\left(S_{1} S_{2}\right)^{2}\right)$.

$E I^{-}$is the identity on $\operatorname{ker}_{\overline{\mathrm{HH}}(A)}(B)=I^{-1} \mathrm{im}(S)$ and applies $B$ to the kernel of (7.1.5), which gives a well-defined map to $\overline{\mathrm{HH}}(A)$ since $B \circ S^{q}=0$ for $q \geq 1$. The image of the latter map is the subspace $\operatorname{ker}(I)=\operatorname{im}(B)$ of $\overline{\mathrm{EHH}}^{\prime \wedge}(A)=\bigoplus_{q \geq 0} I^{-1} \mathrm{im} S^{q+1}$. Then, on the quotient (7.1.5), $E I^{-}$applies $\left(S_{1} S_{2}\right)^{m} u^{-m}$ to $u^{m} \operatorname{ker}\left(S_{1} S_{2}\right)^{m+1}$ for $m \geq 1$, which gives a well-defined map to $\overline{\mathrm{HH}}(A) / \operatorname{ker}(I) \cong \operatorname{im}(I) \subseteq \overline{\mathrm{HC}}(A)$, with image equal to $\operatorname{im}_{\overline{\mathrm{HD}}(A)}\left(S_{1} S_{2}\right)^{m} \cap \operatorname{ker}_{\overline{\mathrm{HD}}(A)}\left(S_{1} S_{2}\right)$, which is $\operatorname{im}\left(S^{m+1}\right) \cap \operatorname{ker}(S)=\operatorname{im}\left(S^{m+1}\right) \cap \operatorname{im}(I)$ as a subspace of $\operatorname{im}(I) \subseteq \overline{\mathrm{HC}}(A)$.

\section{THE REPRESENTATION FUNCTOR AND EQUIVARIANT DELIGNE COHOMOLOGY}

8.1. Equivariant Deligne cohomology. Let $X$ be an affinet variety equipped with an action of a connected reductive algebraic group $G$ with Lie algebra $\mathfrak{g}$. Let act $: \mathfrak{g} \rightarrow \operatorname{Vect}(X)$ be the infinitesimal action and let $\Omega_{X}^{1}$ be the space of Kähler differentials on $X$. Thus, the algebra $\Omega_{X}=\wedge_{\mathbf{k}[X]}\left(\Omega_{X}^{1}\right)$

\footnotetext{
${ }^{1}$ It is possible to generalize everything below to the case of not necessarily affine varieties by replacing the space of global differential forms on $X$ by the sheaf of differential forms.
} 
of algebraic differential forms on $X$ acquires a natural structure of $\mathfrak{g}$-module. One also has a natural $\mathfrak{g}$-action on $\mathbf{k}[\mathfrak{g}]$, a polynomial algebra, induced by the adjoint $\mathfrak{g}$-action on $\mathfrak{g}$ itself. We let $\mathfrak{g}$ act diagonally on the algebra $\Omega_{X}[\mathfrak{g}]:=\Omega_{X} \otimes \mathbf{k}[\mathfrak{g}]$.

Recall that the Cartan model of the equivariant (algebraic) de Rham complex of $X$ is defined as

$$
\left(\left(\Omega_{X}[\mathfrak{g}]\right)^{\mathfrak{g}}, \mathrm{d}_{X}-i_{\mathfrak{g}}\right)
$$

where $\mathrm{d}_{X}$ is the de Rham differential on $X$ and $i_{\mathfrak{g}}$ is the equivariant differential, given by

$$
i_{\mathfrak{g}}(\mathrm{d} f)(x)=\operatorname{act}(x)(f),\left.\quad i_{\mathfrak{g}}\right|_{\Omega_{X}^{0}}=0=\left.i_{\mathfrak{g}}\right|_{\mathbf{k}[\mathfrak{g}]} .
$$

By analogy with cyclic homology, we introduce three new versions of the Cartan model, all equipped with the differential $i_{\mathfrak{g}}-u \mathrm{~d}_{X}$, where $u$ is an extra parameter:

$$
\operatorname{CC}_{\mathfrak{g}}^{\text {per }}(X):=\left(\Omega_{X}[\mathfrak{g}]\right)^{\mathfrak{g}}((u)), \quad \mathrm{CC}_{\mathfrak{g}}(X):=\left(\Omega_{X}[\mathfrak{g}]\right)^{\mathfrak{g}} \otimes R, \quad \mathrm{CC}_{\mathfrak{g}}^{-}(X):=\left(\Omega_{X}[\mathfrak{g}]\right)^{\mathfrak{g}} \llbracket u \rrbracket .
$$

Remark 8.1.3. The complex $\left(\mathrm{CC}_{\mathfrak{g}}^{\text {per }}(X), i_{\mathfrak{g}}-u \mathrm{~d}_{X}\right)$ is isomorphic, up to sign in the differential, to the standard version (8.1.1) tensored by $\mathbf{k}((u))$, via the map $\mathfrak{g}^{*} \stackrel{\sim}{\rightarrow} u^{-1} \mathfrak{g}^{*}, g \mapsto u^{-1} g$.

We define the homological grading $\mathrm{CC}_{\mathfrak{g}}(X)=\oplus_{n} \mathrm{CC}_{\mathfrak{g}}^{n}(X)$ by placing the space $\left(\Omega_{X}^{p} \otimes \mathbf{k}[\mathfrak{g}]\right)^{\mathfrak{g}} \cdot u^{-r}$ in degree $n=p+2 r$, for every $p$ and $r$. Thus, the tensor factor $\mathbf{k}[\mathfrak{g}]$, is assigned homological degree zero, and $u$ has homological degree $|u|=-2$. Each of the differentials $u \mathrm{~d}_{X}$ and $i_{\mathfrak{g}}$, and hence also $i_{\mathfrak{g}}-u \mathrm{~d}_{X}$, has homological degree $(-1)$. We define equivariant Deligne cohomology, which will serve as the natural receptacle of cyclic homology under the representation functor, by

$$
{ }^{\mathcal{D}} H_{\mathfrak{g}}^{\bullet}(X):=H\left(\mathrm{CC}_{\mathfrak{g}}^{\bullet}(X), i_{\mathfrak{g}}-u \mathrm{~d}\right) .
$$

Periodic and negative versions are defined in an obvious way.

Similarly to $\$ 5.5$, we also introduce an internal grading $\mathrm{CC}_{\mathfrak{g}}(X)=\oplus_{k \in \mathbb{Z}} \mathrm{CC}_{\mathfrak{g}}(X)_{k}$, by assigning the space $\left(\Omega_{X}^{p} \otimes \mathbf{k}^{q}[\mathfrak{g}]\right)^{\mathfrak{g}} \cdot u^{-r}$ internal degree $p+q+r$, for every $p, q$, and $r$. Here, we use the notation $\mathbf{k}^{q}[\mathfrak{g}]$ for the space of degree $q$ homogeneous polynomials on $\mathfrak{g}$. Each of the differentials $u \mathrm{~d}_{X}$ and $i_{\mathfrak{g}}$ preserves the internal grading. Therefore, the complex $\left(\mathrm{CC}_{\mathfrak{g}}(X), i_{\mathfrak{g}}-u \mathrm{~d}_{X}\right)$ breaks up into a direct sum over all $k \in \mathbb{Z}$ of the subcomplexes $\left(\mathrm{CC}_{\mathfrak{g}}(X)_{k}, i_{\mathfrak{g}}-u \mathrm{~d}_{X}\right)$.

For $\ell \geq 0$ and $m \in \mathbb{Z}$, we define the weight $m$ component of ${ }^{\mathcal{D}} H_{\mathfrak{g}}^{\ell}(X)$, cf. \&5.5, by

$$
{ }^{\mathcal{D}} H_{\mathfrak{g}}^{\ell}(X, m):=H^{\ell}\left(\mathrm{CC}_{\mathfrak{g}}(X)_{\ell-m}, i_{\mathfrak{g}}-u \mathrm{~d}_{X}\right) .
$$

The natural multiplication in the algebra $\Omega_{X}((u)) \otimes \mathbf{k}[\mathfrak{g}]$ induces a canonical $\left(\mathbf{k}[\mathfrak{g}]^{\mathfrak{g}}\right)[u]$-module structure on the equivariant Deligne cohomology. The module structure and the gradings are related by

$$
\left(\mathbf{k}^{q}[\mathfrak{g}]^{\mathfrak{g}}\right) u^{r} \otimes \mathcal{D}^{\mathcal{D}} H_{\mathfrak{g}}^{\ell}(X, m) \longrightarrow{ }^{\mathcal{D}} H_{\mathfrak{g}}^{\ell-2 r}(X, m-q-r), \quad \forall q, r \geq 0 .
$$

There is also an algebra structure on the equivariant Deligne cohomology, similar to the one on the ordinary Deligne cohomology. In the equivariant case, the product is defined by the formula $\omega \cdot \omega^{\prime}=\omega \cup u^{-1}\left(i_{\mathfrak{g}}-u \mathrm{~d}_{X}\right) \omega^{\prime}$. Here $u^{-1}\left(i_{\mathfrak{g}}-u \mathrm{~d}_{X}\right) \omega^{\prime}$ should be computed by first lifting $\omega^{\prime}$ to $\left(\Omega_{X}[\mathfrak{g}]\right)^{\mathfrak{g}} \otimes \mathbf{k}((u))$, then after performing the operation, projecting back to $\left(\Omega_{X}[\mathfrak{g}]\right)^{\mathfrak{g}} \otimes R$. Compare this with [Lo, §3.6.2] regarding usual Deligne cohomology. (Note that, when $\omega^{\prime}$ is a cocycle, i.e., $\left(i_{\mathfrak{g}}-u \mathrm{~d}_{X}\right) \omega^{\prime}=0$ in $\mathrm{CC}_{\mathfrak{g}}^{\cdot}(X)$, then the above formula can be rewritten as $\omega \cup-\mathrm{d}_{X} \omega_{0}^{\prime}$, where $\omega_{0}^{\prime}$ is the constant term in $u$ of $\omega^{\prime}$.) The algebra structure will not play a role below.

Equivariant Deligne cohomology is functorial with respect to $G$-equivariant maps.

Example 8.1.5. If $\mathfrak{g}$ acts trivially on $X$, we obtain $\mathrm{CC}_{\mathfrak{g}}(X) \cong\left(\Omega_{X} \otimes R, u \mathfrak{d}\right) \otimes \mathbf{k}[\mathfrak{g}]^{\mathfrak{g}}$. The cohomology of the first term on the RHS is

$$
H\left(\Omega_{X} \otimes R, u \mathrm{~d}\right) \cong \Omega_{X} / \mathrm{d} \Omega_{X} \bigoplus\left(H_{\mathrm{DR}}(X) \otimes u^{-1} \cdot \mathbf{k}\left[u^{-1}\right]\right) .
$$


We find that the group ${ }^{\mathcal{D}} H_{\mathfrak{g}}^{\ell}(X, m)$ vanishes for $m>\left[\frac{\ell}{2}\right]$, and

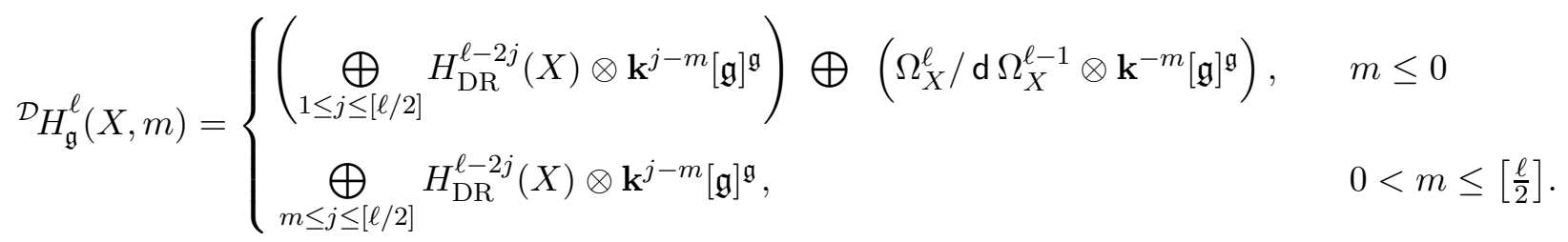

In total degree $\ell$, we therefore obtain ${ }^{\mathcal{D}} H_{\mathfrak{g}}^{\ell}(X) \cong \mathcal{D}^{\mathcal{D}} H^{\ell}(X) \otimes \mathbf{k}[\mathfrak{g}]^{\mathfrak{g}}$, where we write

$$
{ }^{\mathcal{D}} H^{\ell}(X):=\bigoplus_{1 \leq j \leq[\ell / 2]} H_{\mathrm{DR}}^{\ell-2 j}(X) \oplus \Omega_{X}^{\ell} / \mathrm{d} \Omega_{X}^{\ell-1}
$$

for the even or odd-degree part of the usual Deligne cohomology of $X$ for the integer $\ell+1$ shifted down in degree by one. The Deligne cohomology is denoted ${ }^{\mathcal{D}} \tilde{\mathrm{H}}(X, \mathbb{Z}(n+1))$ in [Lo, §3.6].

Example 8.1.6. Let $p: X \rightarrow Y$ be a principal $G$-bundle on an affine variety $Y$, the latter being viewed as a $G$-variety with the trivial $G$-action. Then, analogously to the case of usual equivariant cohomology, there is a canonical isomorphism

$$
\mathcal{D}_{H_{\mathfrak{g}}}(X) \cong{ }^{\mathcal{D}} H^{\bullet}(Y) \text {. }
$$

To prove this, one observes first that a $G$-bundle on an affine variety admits an algebraic connection. Using a connection, one shows that the pull-back morphism $p^{*}: \Omega_{Y} \rightarrow \Omega_{X}$ induces an isomorphism $\mathbf{k}[X] \otimes_{\mathbf{k}[Y]} \Omega_{Y} \stackrel{\sim}{\rightarrow} H\left(\Omega_{X}[\mathfrak{g}], i_{\mathfrak{g}}\right)$. Taking $G$-invariants on each side and using that $G$ is connected and reductive, we deduce an isomorphism $\Omega_{Y}=\left(\mathbf{k}[X] \otimes_{\mathbf{k}[Y]} \Omega_{Y}\right)^{\mathfrak{g}} \stackrel{\sim}{\rightarrow} H\left(\left(\Omega_{X}[\mathfrak{g}]\right)^{\mathfrak{g}}, i_{\mathfrak{g}}\right)$. The required isomorphism ${ }^{\mathcal{D}} H_{\mathfrak{g}}(X) \cong{ }^{\mathcal{D}} H^{\bullet}(Y)$ then follows from the spectral sequence of a double complex, by taking homology of the differential $i_{\mathfrak{g}}$ first.

8.2. The representation functor. Associated with an algebra $A$ and $n \geq 1$, there is an affine scheme $\operatorname{Rep}_{n} A$ that parametrizes $n$-dimensional representations of $A$. Its closed points are algebra homomorphisms $\rho: A \rightarrow \operatorname{Mat}_{n}(\mathbf{k}), a \mapsto \rho(a)$. The scheme $\operatorname{Rep}_{n} A$ comes equipped with a natural action of $\mathrm{GL}_{n}$, the general linear group, by base change transformations. We write $\mathfrak{g l}_{n}=\operatorname{Lie} \mathrm{GL}_{n}$ for the corresponding Lie algebra.

Below, we will use simplified notation: $\operatorname{Rep}_{n}=\operatorname{Rep}_{n} A, \mathrm{DR}_{t}=\mathrm{DR}_{t} A$, etc. The connection between $\mathrm{DR}_{t}$ and the $\mathrm{GL}_{n}$-equivariant de Rham complex of $\operatorname{Rep}_{n}$ is through the evaluation map, which is a 'tautological' homomorphism of algebras,

$$
\text { ev }: A \rightarrow\left(\mathbf{k}\left[\operatorname{Rep}_{n}\right] \otimes \operatorname{Mat}_{n}(\mathbf{k})\right)^{\mathfrak{g l}_{n}}, \quad a \mapsto(\rho \mapsto \rho(a)) .
$$

The above can be defined scheme-theoretically by extending this functorially to representations with coefficients in arbitrary k-algebras; see, e.g., [Gi, §12].

The evaluation map extends uniquely to a homomorphism of $\mathrm{dg}$ algebras,

$$
\text { ev }: \Omega A \rightarrow\left(\Omega_{\operatorname{Rep}_{n}} \otimes \operatorname{Mat}_{n}(\mathbf{k})\right)^{\mathfrak{g l}_{n}}, \quad \operatorname{ev}(\mathrm{d} a)=\operatorname{dev}(a) .
$$

Finally, to extend this to $\Omega_{t} A$, let $\mathrm{ev}(t): \mathfrak{g l}_{n} \rightarrow \operatorname{Mat}_{n}(\mathbf{k})$ be the identity map, viewed as a linear function on $\mathfrak{g l}_{n}$ valued in $\operatorname{Mat}_{n}(\mathbf{k})$, i.e., as an element of the algebra $\mathbf{k}\left[\mathfrak{g l}_{n}\right] \otimes \operatorname{Mat}_{n}(\mathbf{k})$. Put together, we obtain a dg algebra map ev $: \Omega_{t} A \rightarrow\left(\Omega_{\operatorname{Rep}_{n}}\left[\mathfrak{g l}_{n}\right] \otimes \operatorname{Mat}_{n}(\mathbf{k})\right)^{\mathfrak{g l}_{n}}$. Further, taking a trace gives a chain of maps

$$
\Omega_{t} A \stackrel{\text { ev }}{\longrightarrow}\left(\Omega_{\operatorname{Rep}_{n}}\left[\mathfrak{g l}_{n}\right] \otimes \operatorname{Mat}_{n}(\mathbf{k})\right)^{\mathfrak{g l}_{n}} \stackrel{\mathrm{Id} \otimes \text { trace }^{\longrightarrow}}{\longrightarrow}\left(\Omega_{\operatorname{Rep}_{n}}\left[\mathfrak{g l}_{n}\right] \otimes \mathbf{k}\right)^{\mathfrak{g l}_{n}}=\left(\Omega_{\operatorname{Rep}_{n}}\left[\mathfrak{g l}_{n}\right]\right)^{\mathfrak{g l}_{n}} .
$$

The resulting composite map descends to a linear map

$$
\operatorname{tr} \circ \mathrm{ev}: \mathrm{DR}_{t} \underset{33}{\longrightarrow}\left(\Omega_{\operatorname{Rep}_{n}}\left[\mathfrak{g l}_{n}\right]\right)^{\mathfrak{g l}_{n}} .
$$


Then, it is not difficult to verify

Theorem 8.2.1 ([GS], Theorem 6.2.5). For all $n \geq 1$, the map $\operatorname{tr} \circ$ ev induces a map of bicomplexes

$$
\operatorname{tr} \circ \mathrm{ev}:\left(\mathrm{DR}_{t}, \mathrm{~d}, \mathrm{i}_{t}\right) \longrightarrow\left(\left(\Omega_{\operatorname{Rep}_{n}}\left[\mathfrak{g l}_{n}\right]\right)^{\mathfrak{g l}_{n}}, \mathrm{~d}_{\operatorname{Rep}_{n}}, i_{\mathfrak{g l}_{n}}\right)
$$

8.3. From cyclic to equivariant Deligne cohomology. Let us define reduced versions of the previous equivariant complexes and cohomology groups of $X$ by replacing $\Omega_{X}$ by $\bar{\Omega}_{X}=\Omega_{X} / \mathbf{k}$ (where $\mathbf{k} \subseteq \Omega_{X}$ are the scalars in degree zero), and leaving everything else the same. We will put lines over everything to denote the reduced versions.

By Theorem 8.2.1 and construction, we deduce that the map $\operatorname{tr} \circ$ ev induces, for all $n \geq 1$, a canonical morphism of $\mathbf{k}\left[\mathfrak{g l}_{n}\right]^{\mathfrak{g l}_{n}}[u]$-modules:

$$
\overline{\mathrm{EHC}}(A)=H\left(\overline{\mathrm{DR}}_{t} \otimes R, \mathrm{i}_{t}-u \mathrm{~d}\right) \longrightarrow \mathcal{D}_{\bar{H}_{\mathfrak{g l}_{n}}}\left(\operatorname{Rep}_{n}\right)=H\left(\left(\bar{\Omega}_{\mathrm{Rep}_{n}}\left[\mathfrak{g l}_{n}\right]\right)^{\mathfrak{g l}_{n}} \otimes R, i_{\mathfrak{g l}_{n}}-u \mathrm{~d}_{\operatorname{Rep}_{n}}\right) .
$$

The main result of this section is an analogue of the above for the usual cyclic homology:

Theorem 8.3.2. (i) For every $n \geq 1$, one has canonical maps given by the composition

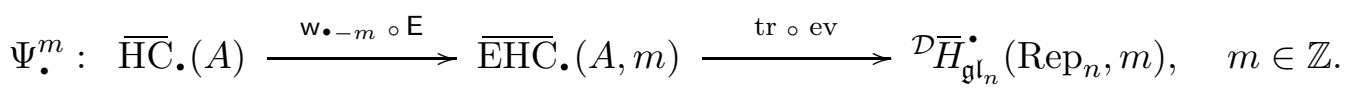

(ii) For $m=0$ and every $\ell \geq 0$, the composite map

$$
\Psi_{\ell}^{\prime}: \operatorname{ker}\left(\mathrm{i}: \overline{\mathrm{DR}}^{\ell} / \mathrm{d} \overline{\mathrm{DR}}^{\ell-1} \rightarrow \bar{\Omega}^{\ell-1} / \mathrm{d} \bar{\Omega}^{\ell-2}\right) \stackrel{\text { 3.1.5 }}{=} \overline{\mathrm{HC}}_{\ell}(A) \stackrel{\Psi_{\ell}^{0}}{\longrightarrow}{ }^{\mathcal{D}} \bar{H}_{\mathfrak{g l}_{n}}^{\cdot}\left(\operatorname{Rep}_{n}, 0\right)
$$

is given by the explicit formula

$$
\Psi_{\ell}^{\prime}(f)=\frac{1}{\ell !} \sum_{0 \leq j \leq[\ell / 2]} u^{-j} \cdot \operatorname{tr} \circ \mathrm{ev} \circ\left(\tilde{\mathrm{d}}^{-1} \mathrm{i}_{t}\right)^{j}(f) .
$$

Remark 8.3.4. (1) In the above formula, $\operatorname{tr} \circ \mathrm{ev} \circ\left(\tilde{\mathrm{d}}^{-1} \mathrm{i}_{t}\right)^{j}(f) \in \bar{\Omega}_{\operatorname{Rep}_{n}}^{\ell-2 j} \otimes \mathbf{k}^{j}\left[\mathfrak{g l}_{n}\right]$. Thus, the sum in the RHS of (8.3.3) a priori lives in

$$
\left(\bigoplus_{1 \leq j \leq\lfloor\ell / 2\rfloor} u^{-j} \cdot \bar{\Omega}_{\mathrm{Rep}_{n}}^{\ell-2 j} \otimes \mathbf{k}^{j}\left[\mathfrak{g l}_{n}\right] \oplus \bar{\Omega}_{\mathrm{Rep}_{n}}^{\ell} / \mathrm{d} \bar{\Omega}_{\mathrm{Rep}_{n}}^{\ell-1}\right)^{\mathfrak{g l}_{n}} .
$$

One checks further that this sum is, in fact, annihilated by the differential $i_{\mathfrak{g l}_{n}}-u \mathrm{~d}_{\mathrm{Rep}_{n}}$, and hence gives a well defined class in ${ }^{\mathcal{D}} \bar{H}_{\mathfrak{g l}_{n}}^{\cdot}\left(\operatorname{Rep}_{n}, 0\right)$.

(2) Note that formula (8.3.3), unlike the definition of the map $\Psi_{\bullet}^{m}$ in the general case of an arbitrary $m$, does not involve the Tsygan map $\mathrm{E}$.

Proof of Theorem 8.3.2 Part (i) follows from (8.3.1), by separating individual homogeneous components and using isomorphism (5.5.3). To prove (ii), we use diagram (5.6.4) and Proposition 5.7.1. In more detail, we consider the sequence of maps

$$
\begin{aligned}
\operatorname{ker}\left(\mathrm{i}: \overline{\mathrm{DR}}^{\ell} / \mathrm{d} \overline{\mathrm{DR}}^{\ell-1} \rightarrow \bar{\Omega}^{\ell-1} / \mathrm{d} \bar{\Omega}^{\ell-2}\right) \stackrel{\text { (3.1.5) }}{=} H^{\ell}(\bar{\Omega} \otimes R, \mathrm{~b}-u \mathrm{~B}) \stackrel{\text { 3.1.8) }}{=} H^{\ell}(\bar{\Omega} / \mathrm{B} \bar{\Omega}, \mathrm{b}) \\
\stackrel{\mathrm{E}^{0}}{\cong} H\left(\overline{\mathrm{DR}}_{t} / \mathrm{d} \overline{\mathrm{DR}}_{t}, \mathrm{i}_{t}\right)^{\ell, 0} \stackrel{\eta}{\longrightarrow} H^{\ell}\left(\left(\overline{\mathrm{DR}}_{t}, \mathrm{i}_{t}-u \mathrm{~d}\right)_{\ell}\right) \stackrel{\operatorname{tr} \circ \mathrm{ev}}{\longrightarrow} \mathcal{D}_{\mathrm{gl}_{n}}^{\ell}\left(\operatorname{Rep}_{n}, 0\right),
\end{aligned}
$$

where the map $\eta$ is given by the formula $\eta=\left(\operatorname{Id}-u^{-1} \tilde{\mathrm{d}}^{-1} \mathrm{i}_{t}\right)^{-1}=\sum_{j} u^{-j} \cdot\left(\tilde{\mathrm{d}}^{-1} \mathrm{i}_{t}\right)^{j}$.

Observe first that, for $f \in \bar{\Omega}^{\ell}$, the component of $\mathrm{E}(f)$ of degree zero in $t$ equals $\frac{1}{\ell !} \cdot f_{\natural}$ (one can see this either directly from formula (5.3.1) or from Lemma 5.3.2). Therefore, the restriction of the map $\mathrm{E}^{0}$ to $H^{\ell}(\bar{\Omega} / \mathrm{B} \bar{\Omega}, \mathrm{b})$ is essentially the map $f \mapsto \frac{1}{\ell !} \cdot f_{\natural}$. It follows that the composition of the first four isomorphisms in (8.3.5) is the isomorphism from (5.5.8) times $\frac{1}{\ell !}$. We deduce that the composite of all the maps in (8.3.5) equals the map given by formula (8.3.3). 
On the other hand, the map $\eta$ provides, thanks to Proposition 5.7.1, an inverse to the isomorphism $\overline{\mathrm{EHC}}_{\ell}(A, 0) \stackrel{\sim}{\rightarrow} H\left(\overline{\mathrm{DR}}_{t} / \mathrm{d} \overline{\mathrm{DR}}_{t}, \mathrm{i}_{t}\right)^{\ell, 0}$, in (5.5.3). Therefore, from the commutativity of diagram (5.6.4) we deduce that the composite of the second, third, and fourth maps in (8.3.5) is equal to the map $\mathrm{w}_{\ell} \circ \mathrm{E}: H^{\ell}(\bar{\Omega} \otimes R, \mathrm{~b}-u \mathrm{~B}) \rightarrow \overline{\mathrm{EHC}}_{\ell}(A, 0)$. We conclude that the composite of the last four maps in (8.3.5) equals the map $\Psi_{\ell}^{0}$, completing the proof.

One can also restrict the map (8.3.1) to the Karoubi-de Rham cohomology $\overline{\mathrm{HD}}(A) \subseteq \overline{\mathrm{HC}}(A)$ and $\overline{\operatorname{EHD}}(A) \subseteq \overline{\operatorname{EHC}}(A)$. This will land in ${ }^{\mathcal{D}} \bar{H}_{\mathrm{cl}_{, \mathfrak{g l}} l_{n}}\left(\operatorname{Rep}_{n}(A)\right)$, the cohomology of the complex

$$
\overline{\mathrm{CC}}_{\mathrm{cl}, \mathfrak{g l}_{n}}\left(\operatorname{Rep}_{n}\right):=\left(\bar{\Omega}_{\mathrm{cl}^{\prime}, \operatorname{Rep}_{n}}^{\mathfrak{l l}_{n}} \bigoplus\left(\bar{\Omega}_{\operatorname{Rep}_{n}} \otimes \mathbf{k}\left[\mathfrak{g l}_{n}\right]_{+}\right)^{\mathfrak{g l}_{n}} \otimes R, \quad \mathrm{i}_{\mathfrak{g l}_{n}}-u \mathrm{~d}_{\mathrm{Rep}_{n}}\right),
$$

where $\bar{\Omega}_{\mathrm{cl}, \operatorname{Rep}_{n}}^{\mathfrak{g l}_{n}}$ denotes the subspace of closed forms and $\mathbf{k}\left[\mathfrak{g l}_{n}\right]_{+} \subset \mathbf{k}\left[\mathfrak{g l}_{n}\right]$ denotes the augmentation ideal. Let $\overline{\mathrm{CC}}_{\mathrm{cl}, \mathfrak{g l}_{n}}^{\wedge}\left(\operatorname{Rep}_{n}\right)$ be the corresponding $\mathbf{k}\left[\mathfrak{g l}_{n}\right]_{+}$-adic completion.

Corollary 8.3.6. By restriction, we obtain maps

$$
\overline{\operatorname{EHD}}(A) \rightarrow H\left(\overline{\mathrm{CC}}_{\mathrm{cl}, \mathfrak{g l}_{n}}\left(\operatorname{Rep}_{n}\right)\right), \quad \text { and } \quad \overline{\mathrm{HD}}(A) \rightarrow H\left(\overline{\mathrm{CC}}_{\mathrm{cl}^{\prime}, \mathfrak{g l}_{n}}^{\wedge}\left(\operatorname{Rep}_{n}\right)\right)
$$

Remark 8.3.7. There are also 'periodic' and 'negative' versions of the above constructions, which produce maps

$$
\overline{\mathrm{EHC}}^{\mathrm{per}, \wedge}(A) \rightarrow H\left(\overline{\mathrm{CC}}_{\mathfrak{g l}_{n}}^{\mathrm{per}, \wedge}\left(\operatorname{Rep}_{n} A\right)\right) \cong \bar{H}_{\mathfrak{g l}_{n}}\left(\operatorname{Rep}_{n} A\right)((u)), \quad \overline{\mathrm{EHC}}^{-, \wedge}(A) \rightarrow H\left(\overline{\mathrm{CC}}_{\mathfrak{g l}_{n}}^{-, \wedge}\left(\operatorname{Rep}_{n} A\right)\right)
$$

Composing with $\mathrm{E}$, we obtain maps

$$
\overline{\mathrm{HC}}^{\text {per }}(A) \rightarrow H\left(\overline{\mathrm{CC}}_{\mathfrak{g l}_{n}, \wedge}^{\text {per }}\left(\operatorname{Rep}_{n} A\right)\right)((u)), \quad \overline{\mathrm{HC}}^{-}(A) \rightarrow H\left(\overline{\mathrm{CC}}_{\mathfrak{g l}_{n}}^{-, \wedge}\left(\operatorname{Rep}_{n} A\right)\right) .
$$

Moreover, we obtain commutative diagrams of commutative squares with exact rows, from (4.4.4) to the version with $\mathrm{E}$ in front of all groups (and with $t$-adic completions), to the commutative square connecting the $\mathbf{k}\left[\mathfrak{g l}_{n}\right]_{+}$-adic completed versions of the above equivariant cohomology groups (and versions one can similarly define analogous to $\overline{\mathrm{HH}}^{\prime}, \overline{\mathrm{HH}}$, and $\overline{\mathrm{HD}}^{\prime}$; note that the equivariant analogue of $\overline{\mathrm{HD}},{ }^{\mathcal{D}} \bar{H}_{\mathrm{cl}, \mathfrak{g}}\left(\operatorname{Rep}_{n}\right)$, was defined above).

8.4. Special cases. The case of $n=1$ : If $n=1$, then $\mathrm{GL}_{1}$ is a 1 -dimensional torus, and $\mathbf{k}\left[\mathfrak{g l}_{1}\right] \cong$ $\mathbf{k}[t]$. Furthermore, since the algebra $\operatorname{Mat}_{1}(\mathbf{k})$ is commutative, the evaluation map for $\bar{\Omega}_{t} A$ may be factored as a composition

$$
\bar{\Omega}_{t} A \stackrel{f \mapsto f_{\natural}}{\longrightarrow} \overline{\mathrm{DR}}_{t} \stackrel{\mathrm{c}_{t}}{\longrightarrow} \overline{\mathrm{DR}}[t] \stackrel{\mathrm{ev} \otimes \mathrm{Id}_{\mathbf{k}[t]}}{\longrightarrow} \bar{\Omega}_{\mathrm{Rep}_{1} A}[t] \otimes \operatorname{Mat}_{1}(\mathbf{k}),
$$

where $c_{t}$ is the map that makes $t$ a central variable, cf. Lemma 5.3.2. Given $f \in \bar{\Omega} A$, we will use simplified notation $\bar{f}:=\operatorname{tr} \circ \operatorname{ev}\left(f_{\natural}\right) \in \bar{\Omega}_{\operatorname{Rep}_{1} A}$. Thus, using the formula of Lemma 5.3.2 and the fact that the trace map yields an algebra isomorphism $\operatorname{tr}: \operatorname{Mat}_{1}(\mathbf{k}) \stackrel{\sim}{\rightarrow} \mathbf{k}$, we deduce

$$
\operatorname{tr} \circ \mathrm{ev} \circ \mathrm{E}(f)=\frac{1}{k !} \cdot \bar{f} \exp (t), \quad \forall f \in \bar{\Omega}^{k} A .
$$

One can use the above formula to obtain an explicit description of the map of Theorem 8.3.2 in the special case that $n=1$ and $m=0$. To this end, observe first that the group $\mathrm{GL}_{1}$ acts trivially on the scheme $\operatorname{Rep}_{1} A$. Therefore, we are in the setting of Example 8.1.5. According to the isomorphism given there, in the special case where $\mathfrak{g}=\mathfrak{g l}_{1}$ and $m=0$, Theorem 8.3.2 yields a map

$$
\Psi_{\ell}^{0}: \overline{\mathrm{HC}}_{\ell}(A) \rightarrow{ }^{\mathcal{D}} \bar{H}^{\ell}\left(\operatorname{Rep}_{1}, 0\right)=\bar{\Omega}_{\mathrm{Rep}_{1} A}^{\ell} / \mathrm{d} \bar{\Omega}_{\operatorname{Rep}_{1} A}^{\ell-1} \oplus\left(\oplus_{j \geq 1} \bar{H}^{\ell-2 j}\left(\operatorname{Rep}_{1} A\right) t^{j} u^{-j}\right)
$$


To compute this map explicitly, we plug formula (8.4.1) for the map E into (8.3.1) and separate the relevant homogeneous components. Then, one finds that the map (8.4.2) is given, for every $\sum_{0 \leq j \leq[\ell / 2]} f_{\ell-2 j} u^{-j}$ representing a class in $\overline{\mathrm{HC}}_{\ell}(A)=H^{\ell}(\bar{\Omega} A \otimes R, \mathrm{~b}-u \mathrm{~B})$, by the formula

$$
\sum_{0 \leq j \leq[\ell / 2]} f_{\ell-2 j} u^{-j} \mapsto \sum_{0 \leq j \leq[\ell / 2]} \frac{1}{(\ell-2 j) !(\ell-2 j) !} \cdot \bar{f}_{\ell-2 j} t^{j} u^{-j}, \quad f_{\ell-2 j} \in \bar{\Omega}^{\ell-2 j} A .
$$

Next, let $A_{\mathrm{ab}}:=A /([A, A])$ be the abelianization of $A$, a quotient of the algebra $A$ by the two-sided ideal generated by the set $[A, A]$. A well known construction in the theory of cyclic homology of commutative algebras produces a map

$$
\overline{\mathrm{HC}}_{\ell}\left(A_{\mathrm{ab}}\right)=H^{\ell}\left(\bar{\Omega} A_{\mathrm{ab}} \otimes R, \mathrm{~b}-u \mathrm{~B}\right) \rightarrow \bigoplus_{j \geq 1} H^{\ell-2 j}\left(\operatorname{Spec} A_{\mathrm{ab}}\right) \oplus \bar{\Omega}_{\mathrm{Spec} A_{\mathrm{ab}}}^{\ell} / \mathrm{d} \bar{\Omega}_{\mathrm{Spec} A_{\mathrm{ab}}}^{\ell-1} .
$$

Specifically, according to [Lo. Proposition 2.3.7], this map is defined by the assignment

$$
\Psi_{\ell}^{\text {classical }}: \sum_{0 \leq j \leq[\ell / 2]} f_{\ell-2 j} u^{-j} \mapsto \bigoplus_{0 \leq j \leq[\ell / 2]} \frac{1}{(\ell-2 j) !} \cdot \bar{f}_{\ell-2 j} .
$$

Let ab $: A \rightarrow A_{\mathrm{ab}}$ be the abelianization homomorphism and write $\mathrm{HC}(\mathrm{ab}): \overline{\mathrm{HC}}(A) \rightarrow \overline{\mathrm{HC}}\left(A_{\mathrm{ab}}\right)$ for the induced map on cyclic homology. The abelianization map $\mathrm{HC}(\mathrm{ab})$ clearly intertwines the Tsygan maps for the algebras $A$ and $A_{\mathrm{ab}}$, respectively. Note further that Hilbert's Nullstellensatz yields canonical isomorphisms $\operatorname{Rep}_{1} A=\operatorname{Rep}_{1} A_{\mathrm{ab}}=\operatorname{Spec} A_{\mathrm{ab}}$. With these observations in mind, comparing formulas (8.4.3) and (8.4.4) yields the comparison result

Corollary 8.4.5. One has $\mathrm{N} ! \circ \Psi^{0}=\Psi^{\text {classical }} \circ \mathrm{HC}(\mathrm{ab})$, where $\mathrm{N} !$ is the map that acts by scalar multiplication by $k$ ! in de Rham degree $k$.

Restricting the map (8.4.2) to $\overline{\mathrm{HD}}_{\ell}(A)$ via the embedding $S_{2}$ from the Connes exact sequence, cf. Theorem 4.1.1, we obtain a map

$$
\Psi_{\ell}^{0} \circ S_{2}: \overline{\operatorname{HD}}_{\ell}(A) \longrightarrow \bigoplus_{j \geq 0} \bar{H}^{\ell-2 j}\left(\operatorname{Rep}_{1} A\right) .
$$

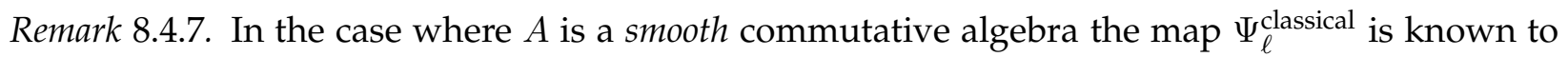
be an isomorphism, see [Lo, Theorem 3.4.12]. It follows that, in this case, each of the maps (8.4.2) and (8.4.6) is an isomorphism as well.

Restriction of $\mathfrak{g l}_{n}$ to scalars: For general $n \geq 1$, the subalgebra of scalar matrices $\mathfrak{g l}_{1} \subseteq \mathfrak{g l}_{n}$ still acts trivially on $\operatorname{Rep}_{n} A$. Thus, restricting the general construction to $\mathfrak{g l}_{1}$ produces a map

$$
\overline{\mathrm{HC}}_{\ell}(A) \rightarrow \bar{\Omega}_{\operatorname{Rep}_{n} A}^{\ell} / \mathrm{d} \bar{\Omega}_{\operatorname{Rep}_{n} A}^{\ell-1} \oplus\left(\oplus_{j \geq 1} \bar{H}^{\ell-2 j}\left(\operatorname{Rep}_{n} A\right)\right) .
$$

There is also a comparison result, similar to Corollary 8.4.5, saying that the map (8.4.8) is equal, up to a twist by the automorphism $\mathrm{N}$ !, to the composition of the evaluation map

$$
\overline{\mathrm{HC}}_{\ell}(A) \rightarrow \overline{\mathrm{HC}}_{\ell}\left(\mathbf{k}\left[\operatorname{Rep}_{n} A\right] \otimes \operatorname{Mat}_{n}(\mathbf{k})\right) \cong \overline{\mathrm{HC}}_{\ell}\left(\mathbf{k}\left[\operatorname{Rep}_{n} A\right]\right)
$$

with isomorphism $\Psi^{\text {classical }}$.

If we don't restrict to scalars, the maps $\overline{\mathrm{HC}}(A) \rightarrow{ }^{\mathcal{D}} \bar{H}_{\mathfrak{g l}_{n}}\left(\operatorname{Rep}_{n} A\right)$ should capture finer information, having to do with the equivariant geometry of the representation scheme.

\section{REFERENCES}

[BKR] Yu. Berest, G. Khachatryan, and A. Ramadoss, Derived representation schemes and cyclic homology, arXiv:1112.1449

[CBEG] W. Crawley-Boevey, P. Etingof, and V. Ginzburg, Noncommutative geometry and quiver algebras, Adv. Math. 209 (2007), no. 1, 274-336, arXiv:math.AG/0502301.

[Con] A. Connes, Noncommutative differential geometry, Inst. Hautes Études Sci. Publ. Math. (1985), no. 62, $257-360$. 
[CQ1] J. Cuntz and D. Quillen, Algebra extensions and nonsingularity, J. Amer. Math. Soc. 8 (1995), no. 2, $251-289$.

[CQ2] _ O O _ O _ _ physics for Raoul Bott (Cambridge, MA) (S.-T. Yau, ed.), Conf. Proc. Lecture Notes Geom. Topology, vol. 4, Internat. Press, Inc., 1995, pp. 77-111.

[FT] B. Feigin and B. Tsygan, Additive K-theory and crystalline cohomology, Funktsional. Anal. i Prilozhen. (Russian) (1985), no. 19, 52-62.

[Gi] V. Ginzburg, Lectures on noncommutative geometry, arXiv:math.AG/0506603. 2005.

[GS] V. Ginzburg and T. Schedler, Free products, cyclic homology, and the Gauss-Manin connection, arXiv:0803.3655: to appear in Adv. Math.

[Go] T. Goodwillie, Cyclic homology, derivations, and the free loopspace. Topology 24 (1985), 187-215.

[LQ] J.-L. Loday and D. Quillen, Cyclic homology and the Lie algebra homology of matrices, Comment. Math. Helv. (1984), no. $59,569-591$.

[Kar] M. Karoubi, Homologie cyclique et K-théorie, Astérisque (1987), no. 149, 147.

[Lo] J.-L. Loday, Cyclic homology, Grundlehren der Mathematischen Wissenschaften, vol. 301, Springer-Verlag, Berlin, 1998.

[Ri] G. Rinehart, Differential forms on general commutative algebras, Trans. Amer. Math. Soc. (1963), no. 108, $195-222$.

[TT] D. Tamarkin and B. Tsygan, The ring of differential operators on forms in noncommutative calculus. 105-131, Proc. Sympos. Pure Math., 73, A.M.S., Providence, RI, 2005.

[Tsy1] B. Tsygan, Homology of matrix Lie algebras over rings and the Hochschild homology, Uspekhi Mat. Nauk (Russian) (1983), no. 38, 217-218.

[Tsy2] , On the morphism from periodic cyclic homology to equivariant cohomology, Appendix to [GS08]. (Preprint 2011, to appear).

V.G.: Department of Mathematics, University of ChicAgo, ChicAgo, IL 60637, USA.

E-mail address: ginzburg@math.uchicago.edu

T.S.: Department of MAthematics, MIT, CAmbridge, MA 02139, USA

E-mail address: traschedegmail.com 